\title{
The role of CRISPLD1 in the transition to heart failure
}

\author{
Dissertation
}

for the award of the degree

"Doctor rerum naturalium"

of the Georg-August-Universität Göttingen

within the doctoral program Molecular Medicine

of the Georg-August University School of Science (GAUSS)

submitted by

Vanessa Hindmarsh, née Kernke

born in Salzgitter

Göttingen, October 2021 


\section{Thesis Committee Members}

Prof. Dr. Bernd Wollnik (first reviewer)

Institute of Human Genetics

University Medical Center Göttingen

Prof. Dr. Dörthe M. Katschinski (second reviewer)

Institute of Cardiovascular Physiology

University Medical Center Göttingen

\section{Dr. George Kensah}

Department of Thoracic and Cardiovascular Surgery

University Medical Center Göttingen

\section{Additional Members of the Examination Board}

Prof. Dr. Katrin Streckfuß-Bömeke

Department of Cardiology and Pneumology

University Medical Center Göttingen

PD Dr. Laura Zélarayan-Behrend

Institute of Pharmacology and Toxicology

University Medical Center Göttingen

Prof. Dr. Ralf Dressel

Institute for Cellular and Molecular Immunology

University Medical Center Göttingen 


\section{Published data}

Parts of the presented data in this doctoral thesis were published as an original research article before the submission date.

Khadjeh, S., Hindmarsh, V., Weber, F., Cyganek, L., Vidal, R.O., Torkieh, S., Streckfuss-Bömeke,

K., Lbik, D., Tiburcy, M., Mohamed, B.A., Bonn, S., Toischer, K. \& Hasenfuss, G. (2020) CRISPLD1: a novel conserved target in the transition to human heart failure. Basic research in cardiology, 115, 27. 
Für meine Tochter Hanna Malina 


\section{Table of contents}

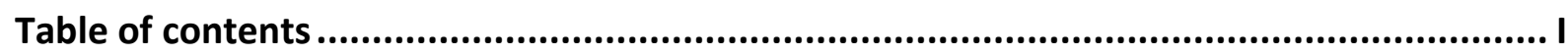

List of figures ................................................................................................................ IV

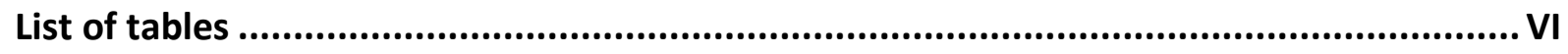

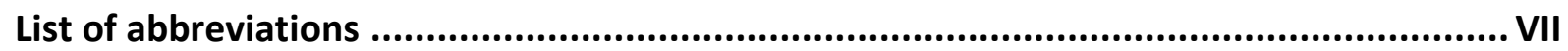

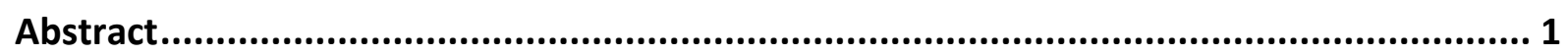

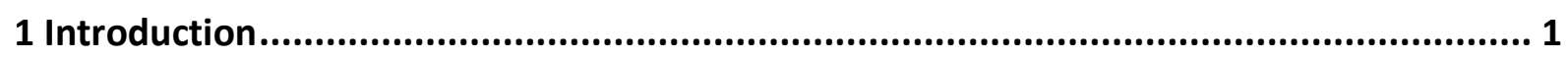

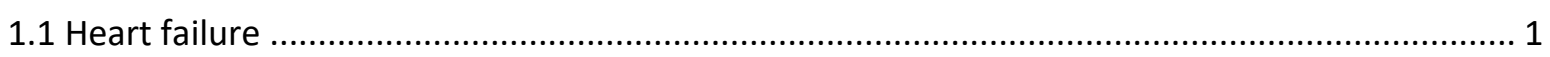

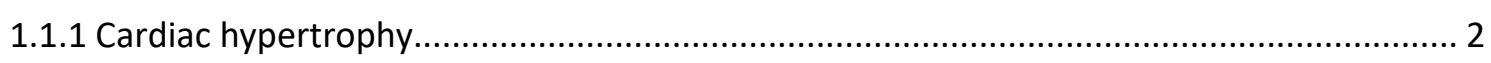

1.1.2 Molecular mechanisms of the transition from cardiac hypertrophy to heart failure ......... 3

1.2 Calcium as a central regulator in the heart and its role in $\mathrm{HF}$ progression ................................. 6

1.3 Transcriptomics of human and mouse during the transition to heart failure ............................. 8

1.4 Cysteine-rich secretory protein LCCL domain-containing 1 (CRISPLD1) ................................... 9

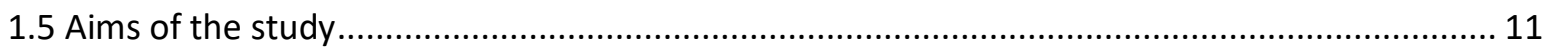

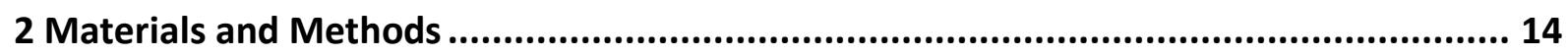

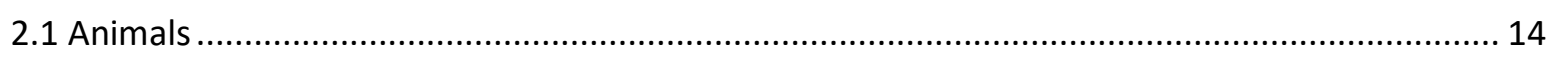

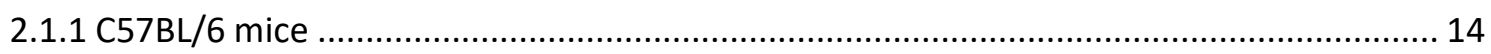

2.1.2 C57BL/6N-Tg(Cag-Flpo)1Afst/Mmcd (CAG-FlpO) mice ..................................................... 14

2.1.3 B6N.FVB(B6)-Tg(Myh6-cre)2182Mds/J (Myh6-Cre) mice .................................................. 14

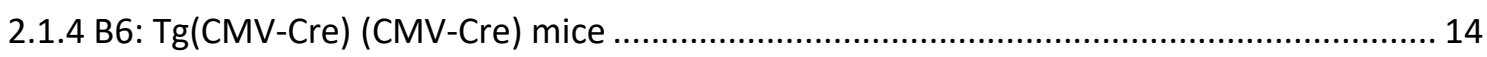

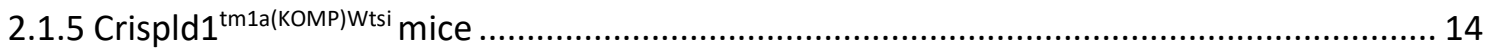

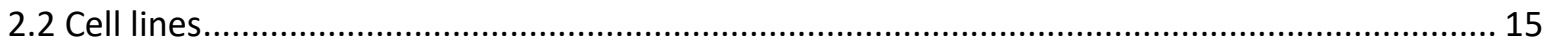

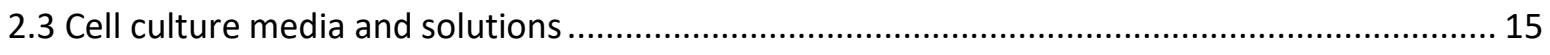

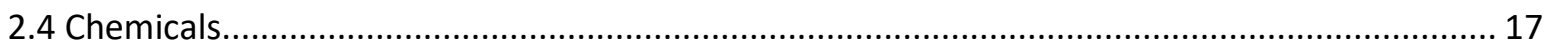

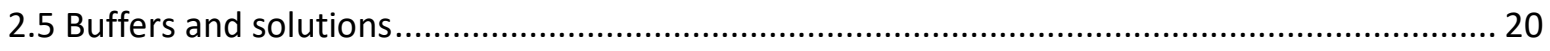

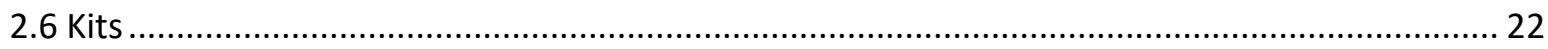

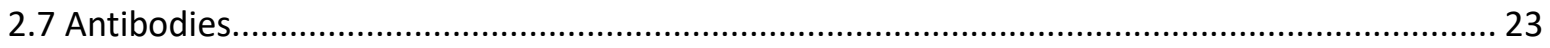

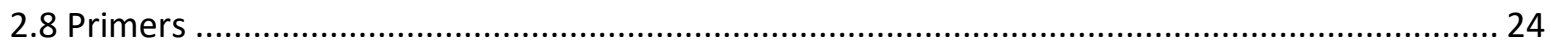

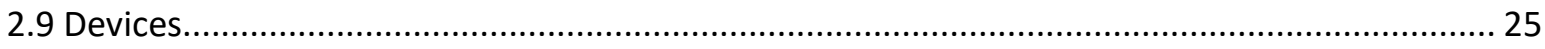

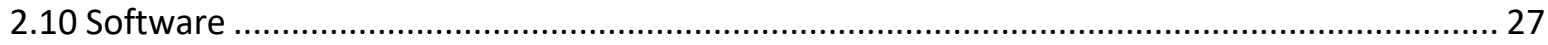

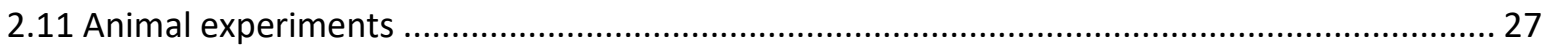

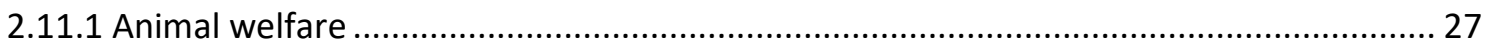

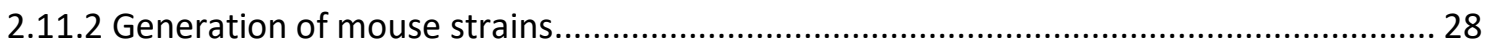

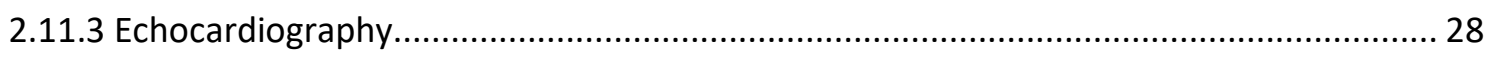

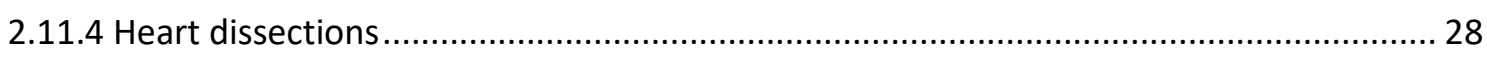




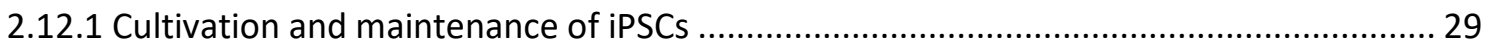

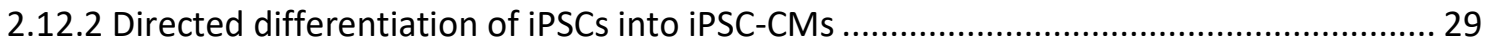

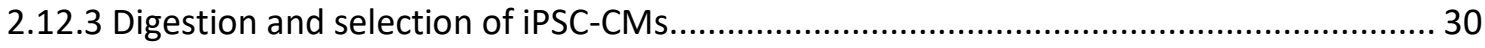

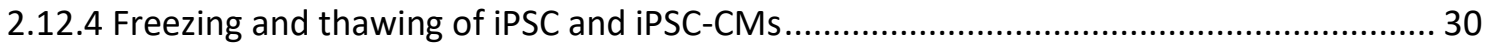

2.12.5 Generation and validation of KO-iPSC clones by CRISPR/Cas9 ................................... 31

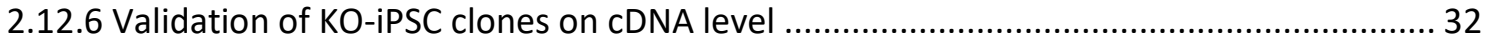

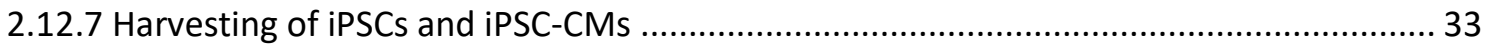

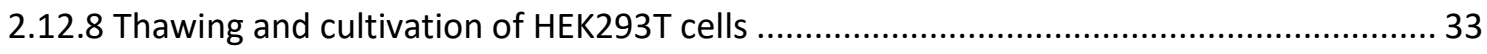

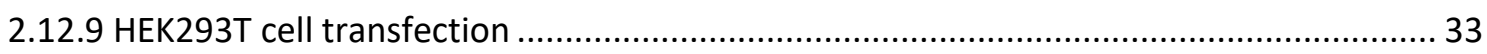

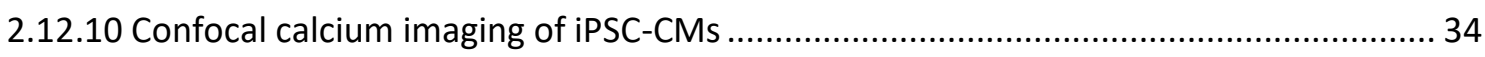

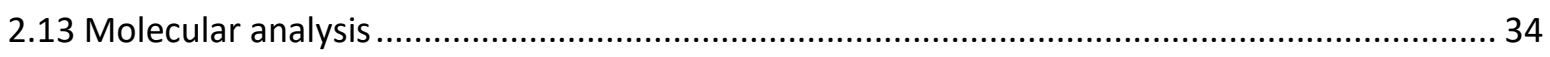

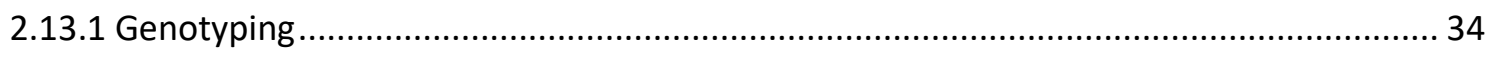

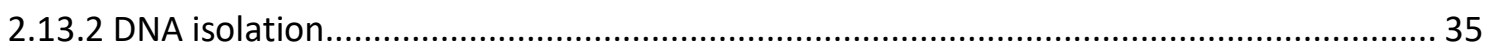

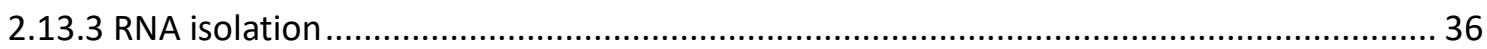

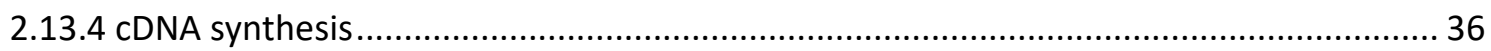

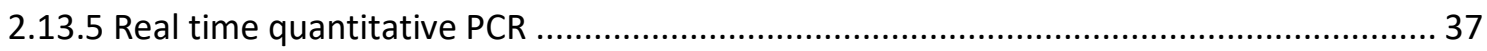

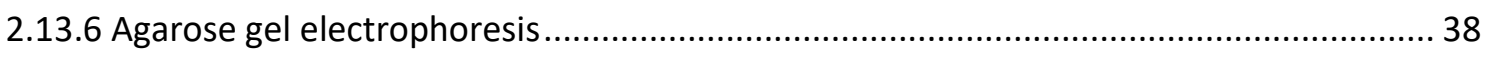

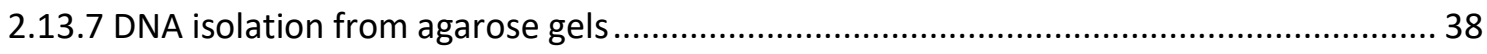

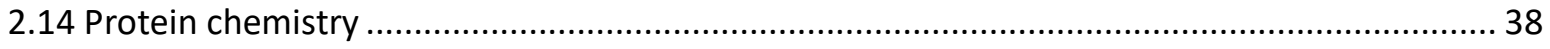

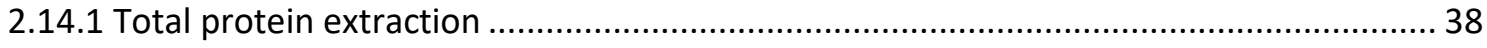

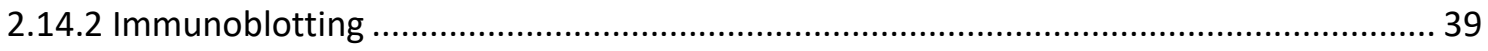

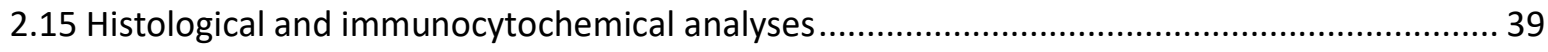

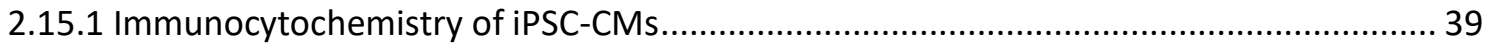

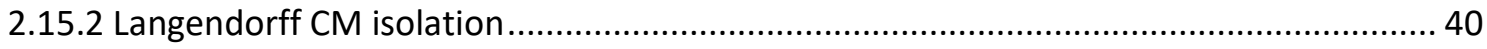

2.15.3 Paraffin embedding, dewaxing and rehydration ............................................... 40

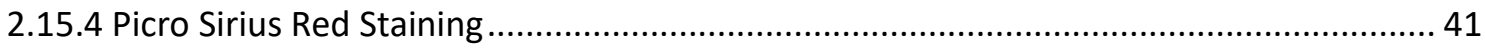

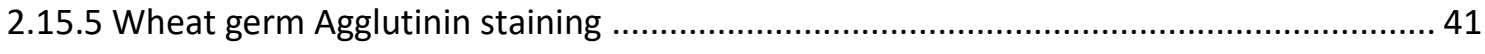

2.15.6 Quantification of histological stainings .......................................................... 41

2.15.7 Epifluorescence calcium-transient measurement of isolated mouse cardiomyocytes ... 41

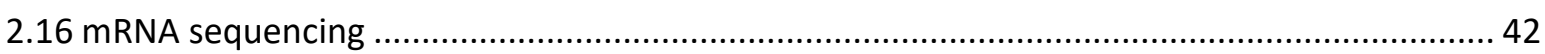

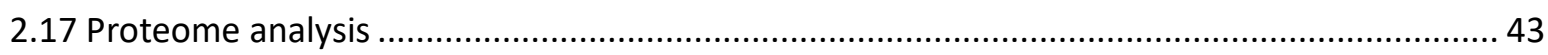

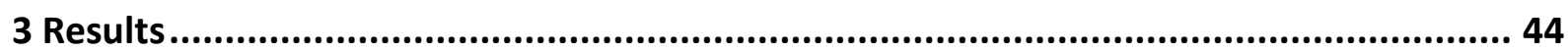

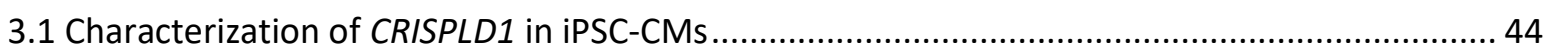

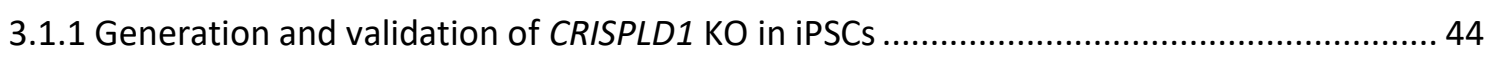

3.1.2 CRISPLD1 KO does not alter sarcomeric structure of iPSC-CMs ...................................45 
3.1.4 CRISPLD1 KO can rescue disturbance of helothermine treated iPSC-CM calcium transients

3.1.5 CRISPLD1 is involved in the regulation of cardiomyopathy, inflammation, calcium regulation and ECM organization.

3.2 The role of Crispld1 in the mouse heart. 53

3.2.1 Generation and validation of Crispld1 $\mathrm{KO}$ mice...

3.2.2 The survival of heart-specific Crispld1 $\mathrm{KO}$ mice is reduced

3.2.3 Heart-specific Crispld1 KO mice show a dilated cardiac phenotype in echocardiography 59

3.2.4 Crispld1 $\mathrm{KO}$ does not influence $\mathrm{CM}$ size, fibrosis or cardiac gene expression in mice 62

3.2.5 Calcium homeostasis is not impaired in Crispld1 KO mouse models 66

4 Discussion 69

4.1 The role of CRISPLD1 in iPSC-CMs 69

4.1.1 The influence of CRISPLD1-deficient iPSC-CMs on the calcium homeostasis.... 70

4.1.2 CRISPLD1 influences hypertrophic, apoptotic and calcium regulating pathways 71

4.1.3 CRISPLD1 has a functional role in the transition to HF

4.2 The role of Crispld1 in the mouse heart.

4.2.1 Generation of Crispld1 KO mice could not be validated in the heart-specific KO line, but showed a downregulated truncated mRNA in the ubiquitous KO mouse line

4.2.2 Heart-specific Crispld1 KO but not ubiquitous Crispld1 KO leads to a dilated cardiac phenotype 75

4.2.3 Molecular analyses cannot explain the dilated heart-phenotype ............................... 78

4.2.4 Crispld1 KO in mice does not influence the cardiac calcium homeostasis ......................79

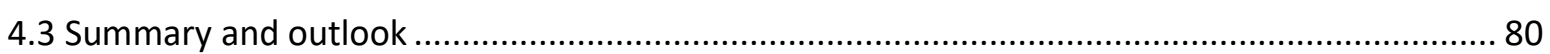

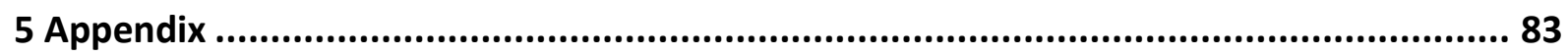

References................................................................................................ 85

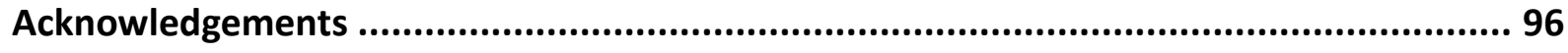

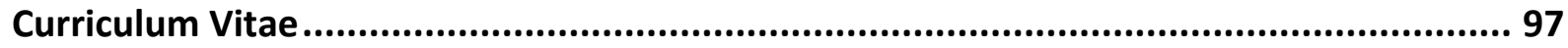




\section{List of figures}

Figure 1-1 Last 28-year prevalence and future trend of HF globally. 1

Figure 1-2 Overview of the transition from normal adult heart to hypertrophy and heart failure........ 3

Figure 1-3 Scheme of selected signaling pathways involved in cardiac hypertrophy and the transition to heart failure. 5

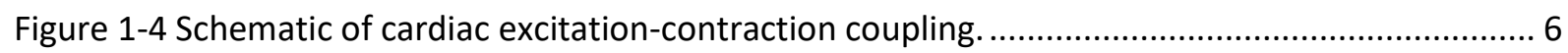

Figure 1-5 Transcriptomics of $\mathrm{PO}$-induced $\mathrm{CH}$ and $\mathrm{HF}$ in human and mouse myocardium.................. 9

Figure 1-6 Protein structure of CRISPLD1 and alignment to helothermine....................................... 10

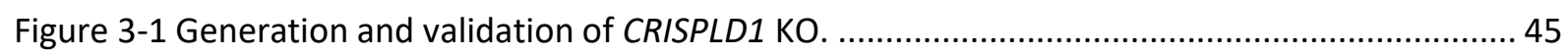

Figure 3-2 CRISPLD1 KO iPSC-CMs show normal cardiac marker expression in IF analysis. ................. 46

Figure 3-3 Functional analysis of calcium transients of CRISPLD1 KO iPSC-CMs and WT iPSC-CMs at day 63-74 by confocal calcium imaging.

Figure 3-4 Concentration-dependent effect of helothermine treatment on WT iPSC-CM calcium transient amplitude.

Figure 3-5 Functional analysis of calcium transients of helothermine treated CRISPLD1 KO iPSC-CMs and WT iPSC-CMs at day 63-74 by confocal calcium imaging.. 48

Figure 3-6 ClueGO analysis of transcriptomics of CRISPLD1 KO iPSC-CMs vs. WT iPSC-CMs. 50

Figure 3-7 DEGs of WT vs. CRISPLD1 KO iPSC-CMs in adrenergic signaling pathway map from KEGG (hsa04261). 51

Figure 3-8 Comparison of transcriptomics and proteomics of CRISPLD1 KO iPSC-CMs vs. WT iPSC-CMs.

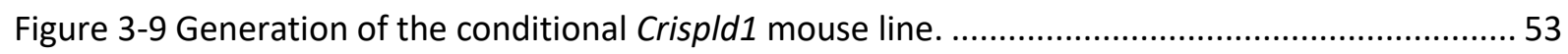

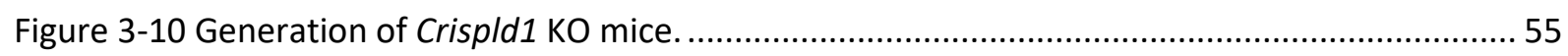

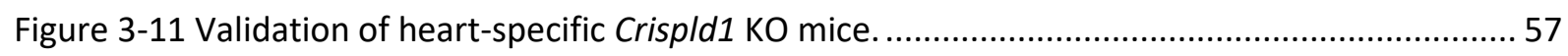

Figure 3-12 Validation of ubiquitous Crispld1 KO mice.................................................................. 58

Figure 3-13 Survival curve of Crispld1 KO mouse lines compared to control. ......................................59

Figure 3-14 Basal echocardiographic phenotyping of Crispld1 KO mouse lines compared to their

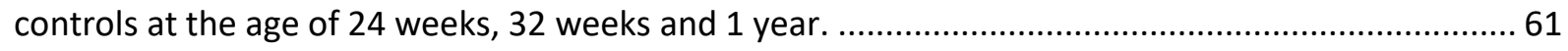

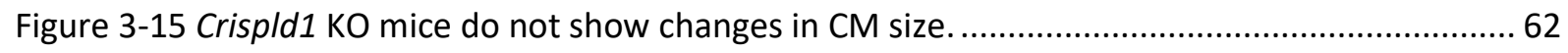

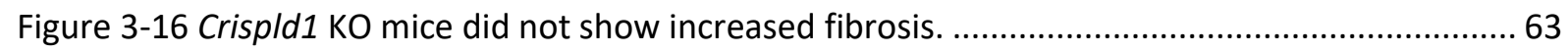

Figure 3-17 Gene expression of cardiac stress markers relative to 185 by RT-qPCR in 25 weeks old

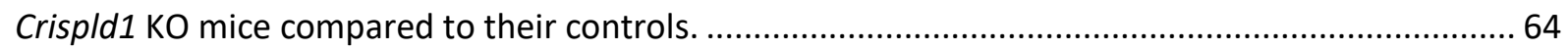

Figure 3-18 Analysis of Camk2a activity in the heart-specific Crispld1 KO mice.................................... 65

Figure 3-19 Analysis of Camk2a activity in the ubiquitous Crispld1 KO mice. ................................... 66 
Figure 3-20 Functional analysis of calcium transients of isolated CMs of Crispld1 KO mouse models at $1 \mathrm{~Hz}, 2 \mathrm{~Hz}$ and $4 \mathrm{~Hz}$. 68

Figure 5-1 Western Blot analysis of four commercially available CRISPLD1 antibodies...................... 83

Figure 5-2 Validation of Myh6xCRISPLD1 KO mice by RT-qPCR. .84

Figure 5-3 CRISPLD1 expression reads in human fetal and adult ventricle, iPSC-CMs and cardiac fibroblasts (CF). 84 


\section{List of tables}

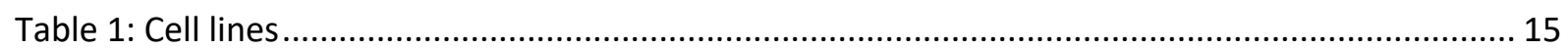

Table 2: Media, supplements, and solutions for cell culture ........................................................... 15

Table 3: Human cell culture medium and solution components ................................................... 16

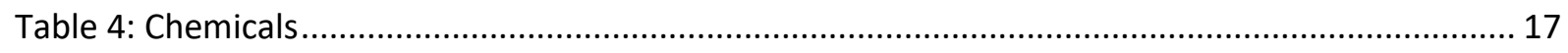

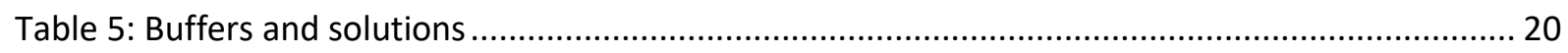

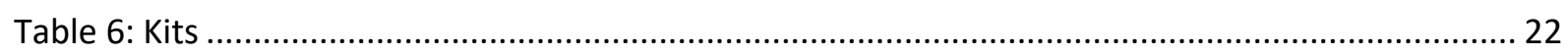

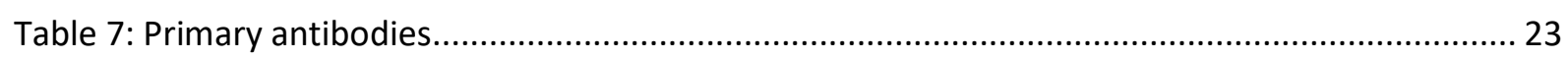

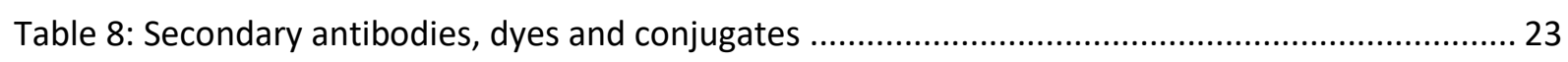

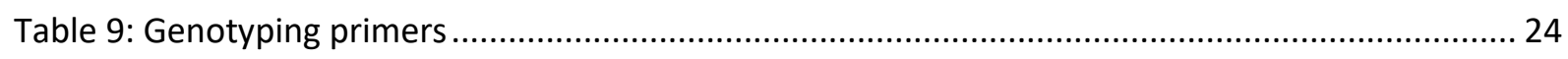

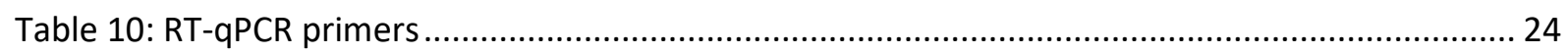

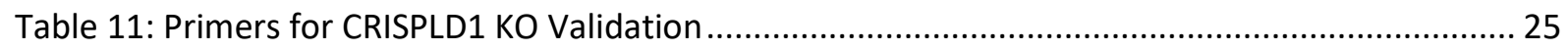

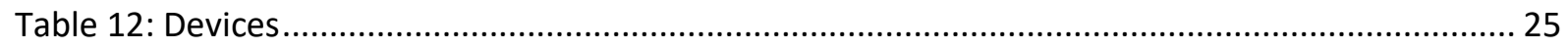

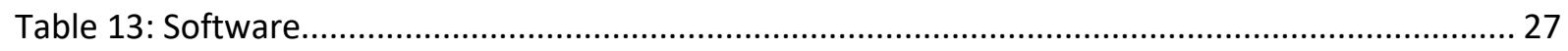

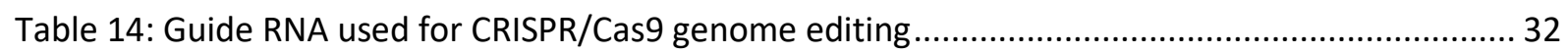

Table 15: Standard PCR reaction for KO clone validation on gDNA level ............................................ 32

Table 16: Standard PCR protocol for KO clone validation on gDNA level ........................................... 32

Table 17: Standard PCR reaction for KO clone validation on CDNA level............................................ 32

Table 18: Standard PCR protocol for KO clone validation on cDNA level ............................................ 33

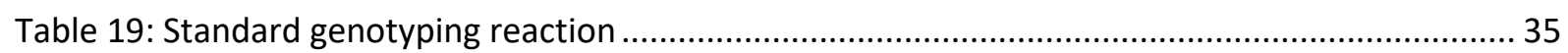

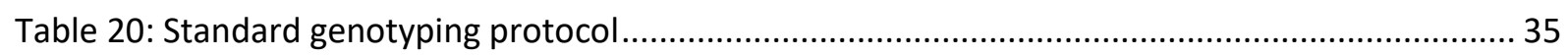

Table 21: RT-qPCR reaction mix for a 384 -well plate ....................................................................... 37

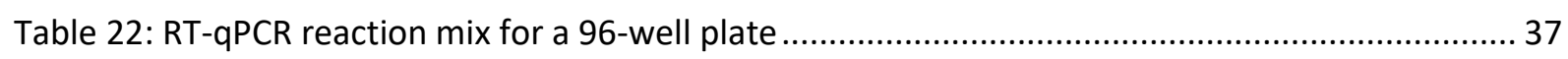

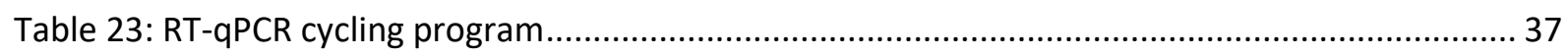




\section{List of abbreviations}

\begin{tabular}{|c|c|c|c|}
\hline ACTN & $\alpha$-Actinin & ERK & Extracellular regulated kinase \\
\hline aa & Amino acid & FBS & Fetal bovine serum \\
\hline AC & Adenylyl cyclase & FKBP12.6 & 12.6 kDa FK506-binding protein \\
\hline ANP & Atrial natriuretic peptide & FS & Fractional shortening \\
\hline ATP & Adenosine triphosphate & GAPDH & $\begin{array}{l}\text { Glyceraldehyde 3-phosphate } \\
\text { dehydrogenase }\end{array}$ \\
\hline ATP2A2 & $\begin{array}{l}\text { Sarcoplasmic/endoplasmic reticulum } \\
\text { calcium ATPase } 2\end{array}$ & GATA4 & GATA binding protein 4 \\
\hline ATR & Angiotensin II receptor & gDNA & Genomic deoxyribonucleic acid \\
\hline BNP & Brain natriuretic peptide & $\mathrm{HCl}$ & Hydrochloric acid \\
\hline bp & Base pairs & HEK & Human embryonic kidney cells \\
\hline BSA & Bovine serum albumin & Helo & Helothermine \\
\hline $\mathrm{Ca}^{2+}$ & Calcium & HEPES & $\begin{array}{l}\text { N-2-Hydroxyethylpiperazine-N'-2- } \\
\text { Ethanesulfonic Acid }\end{array}$ \\
\hline CAMKII & $\begin{array}{l}\text { Calcium/calmodulin-dependent } \\
\text { protein kinase type II }\end{array}$ & HF & Heart failure \\
\hline CAMP & 3'5'-cyclic AMP & iPSC & Induced pluripotent stem cell \\
\hline CaT & Calcium transient & iPSC-CM & $\begin{array}{l}\text { Induced pluripotent stem cell-derived } \\
\text { cardiomyocyte }\end{array}$ \\
\hline cDNA & Complementary DNA & IWP2 & Inhibitor of Wnt productions-2 \\
\hline CF & Cardiac fibroblasts & JNK & JUN N-terminal kinase \\
\hline $\mathrm{CH}$ & Cardiac hypertrophy & kDa & Kilodalton \\
\hline CHD & Coronary heart disease & KO & Knockout \\
\hline CM & Cardiomyocyte & LTCC & Voltage-gated L-type calcium channel \\
\hline CMV & Cytomegalovirus & LV & Left ventricle \\
\hline CRISPLD1 & $\begin{array}{l}\text { Cysteine Rich Secretory Protein LCCL } \\
\text { Domain Containing } 1\end{array}$ & LVAWd & $\begin{array}{l}\text { Diastolic left ventricular anterior wall } \\
\text { thickness }\end{array}$ \\
\hline cTNT & Cardiac troponin $\mathrm{T}$ & LVEDD & Left ventricular end-diastolic diameter \\
\hline Cx43 & Connexin 43 & LVEF & Left ventricular ejection fraction \\
\hline DCM & Dilated cardiomyopathy & МАРККК & MAP kinase kinase kinases \\
\hline DEG & Differentially expressed gene & MEK & $\begin{array}{l}\text { Mitogen-activated protein kinase } \\
\text { kinase }\end{array}$ \\
\hline DMEM & Dulbecco's Modified Eagle Medium & mRNA & Messenger RNA \\
\hline DMSO & Dimethyl sulfoxide & MFD & Minimal fiber diameter \\
\hline EDTA & Ethylenediamine tetraacetic acid & MLC2V & Ventricular myosin light chain-2 \\
\hline EndoR & Endothelin 1 receptor & Myh6 & Myosin heavy chain 6 \\
\hline Myh7 & Myosin heavy chain 7 & ROS & Reactive oxygen species \\
\hline
\end{tabular}




\begin{tabular}{|c|c|c|c|}
\hline NCX & Sodium-calcium exchanger & rpm & Revolutions per minute \\
\hline NF & Non-failing & rpkm & $\begin{array}{l}\text { Reads per kilobase per million mapped } \\
\text { reads }\end{array}$ \\
\hline NFAT & Nuclear factor of activated T-cells & rRNA & Ribosomal RNA \\
\hline nppa & Natriuretic peptide precursor $A$ & RT & Room temperature \\
\hline nppb & Natriuretic peptide precursor B & RT-qPCR & Real-time quantitative PCR \\
\hline NPR & natriuretic peptide receptor & RYR2 & Ryanodine receptor 2 \\
\hline PBS & Phosphate buffered saline & SERCA & Sarcoplasmic reticulum $\mathrm{Ca}^{2+}$ ATPase \\
\hline P-CamK & $\begin{array}{l}\text { Phosphorylated } \mathrm{Ca}^{2+} / \text { calmodulin- } \\
\text { dependent protein kinase }\end{array}$ & SR & Sarcoplasmic reticulum \\
\hline PCR & Polymerase chain reaction & TAC & Transverse aortic constriction \\
\hline PKA & Protein kinase $A$ & TBE & Tris/borate/EDTA buffer \\
\hline PKC $\alpha$ & Protein kinase $\mathrm{C} \alpha$ & TBST & Tris buffered saline with Tween-20 \\
\hline PKG & Protein kinase G & TRIS & Tris(hydroxymethyl)aminomethane \\
\hline PLC & Phospholipase C & TRPC & Transient receptor protein channel \\
\hline PLN & Phospholamban & TZV & Thiazovivin \\
\hline PO & Pressure overload & WB & Western blot \\
\hline RCAN & Calcineurin & WGA & Wheat germ agglutinin \\
\hline RHOA & Transforming protein $\mathrm{RHOA}$ & WT & Wildtype \\
\hline RNA & Ribonucleic acid & $\alpha / \beta-A R$ & $\alpha / \beta$-adrenergic receptor \\
\hline
\end{tabular}




\section{Abstract}

Heart failure is a leading cause of death worldwide. It is defined as a condition in which the heart is unable to pump a sufficient amount of blood through the body to satisfy its oxygen need and can be caused by various cardiac diseases. Although several mechanisms are already identified to play a role in the transition to HF, there is still a lack of knowledge about the precise mechanisms. To gain better insights into these mechanisms, previous studies in the group performed RNA-sequencing of human myocardium samples from patients suffering from aortic stenosis with compensated hypertrophy or heart failure. The obtained dataset was compared to data from analogous progression stages in the transverse aortic constriction mouse model to identify novel target genes in the disease. Among these genes, CRISPLD1 showed conserved expression levels and was upregulated during the transition to heart failure in human and mouse. Protein structure analysis identified a V5/Tpx-1 related conserved site which is known to be evolutionary related to the toxin helothermine, that regulates calcium homeostasis. To elucidate the function of CRISPLD1 in the heart, induced pluripotent stem cell derived cardiomyocytes (iPSC-CMs) and two knockout mouse models were analyzed. CRISPLD1-deficient iPSC-CMs were generated via CRISPR/Cas9 and showed dysregulation of calcium handling. Transcriptome analysis of these cells revealed a downregulation of prohypertrophic, proapoptotic and calcium-regulating pathways, suggesting CRISPLD1 loss-offunction as being beneficial in response to cardiomyocyte stress. However, analysis of Crispld1-deficient knockout mouse lines showed contrary results. While the ubiquitous Crispld1 knockout mouse line did not show any phenotype, the heart-specific Crispld1 knockout mouse line displayed a severe dilated cardiac phenotype with increased mortality. Analysis of hypertrophic markers, measurement of cardiomyocyte hypertrophy or fibrosis did not indicate any alterations. Even more, calcium cycling evaluations of isolated mouse cardiomyocytes could not confirm the findings of iPSC-CMs and again no alterations comparing the mouse lines were observed. Although, the severe cardiac phenotype of heartspecific Crispld1 knockout mice could not be explained, results obtained in cell culture were promising and identified CRISPLD1 as playing a role in calcium cycling in CMs. In conclusion, the findings from this thesis give first insights into the potential role of CRISPLD1 as a calcium regulator in the heart in a human and murine model system. However, future research is necessary to identify its specific role and explain the severe mouse phenotype. 


\section{Introduction}

\subsection{Heart failure}

Cardiovascular diseases are the main cause of death for the last 20 years. According to the WHO, about 9 million people died from heart diseases in 2019, which represents $16 \%$ of total deaths worldwide. Whereas these numbers are increasing globally, deaths due to heart diseases are decreasing in the European region (WHO, 2020).

Within heart diseases, heart failure (HF) is the leading cause of death and more prevalent in the elderly (Dickstein et al., 2008; Verheugt et al., 2010). According to estimates, 64.34 million people are currently facing HF worldwide. Analyzing the prevalence of the last 30 years, an increase of about $36 \%$ obtained which is expected to increase even more until 2030 (Figure 1-1). Therefore, the total medical costs for HF are currently estimated at 346.17 billion US $\$$ and expected to increase to 398.44 billion US \$ by 2030 (Lippi \& Sanchis-Gomar, 2020).

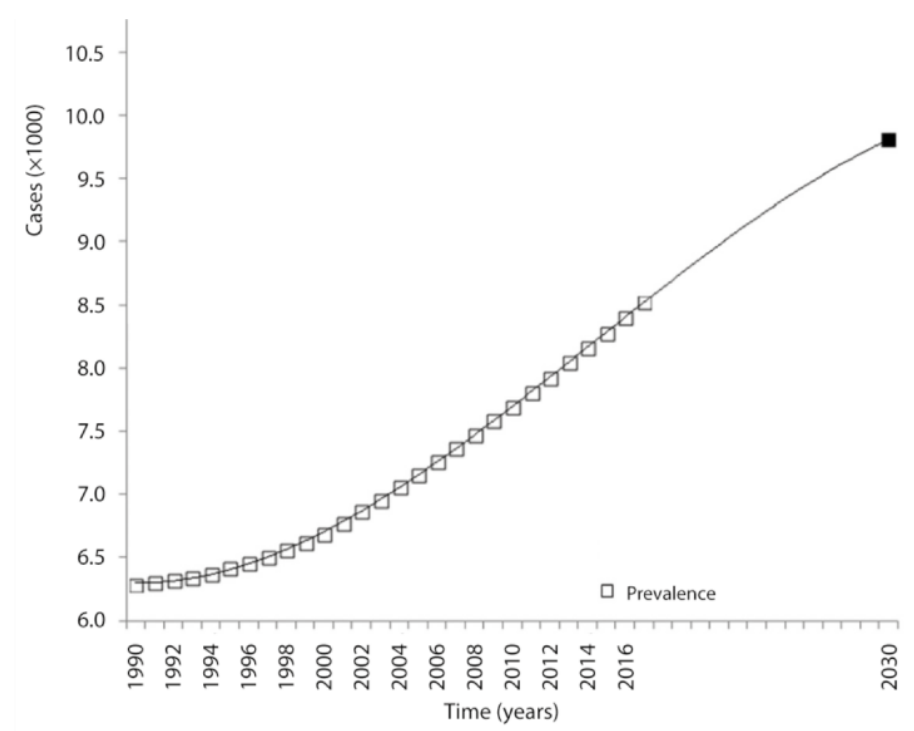

Figure 1-1 Last 28-year prevalence and future trend of HF globally. Since 1990, the prevalence of HF increased about $36 \%$ to 8.52 per 1000 inhabitants worldwide. This rising trend is expected to not change until 2030. Adapted and modified from (Lippi \& Sanchis-Gomar, 2020).

HF is defined as a condition in which the heart is unable to pump a sufficient amount of blood to supply the body with oxygen. Various cardiac conditions and pathologies such as congenital heart diseases, coronary ischemia, chronic hypertension and many more can result in $\mathrm{HF}$ (Inamdar \& Inamdar, 2016; Ziaeian \& Fonarow, 2016; Hajar, 2019). Typical symptoms of HF are shortness of breath, fatigue, oedemas, or pulmonary crackles which are due to structural changes in the heart as a compensatory mechanism to injuries. These compensatory 
mechanisms are characterized by cardiac enlargement, referred as cardiac hypertrophy to maintain normal cardiac function (Grossman et al., 1975; Ponikowski et al., 2016).

\subsubsection{Cardiac hypertrophy}

The heart responses to volume or pressure overload by hypertrophy, to maintain its cardiac function (Grossman et al., 1975; Frey \& Olson, 2003). The most prevalent cells in the heart are cardiomyocytes. Contrary to the other cell types like fibroblasts, endothelial cells, immune cells or progenitor cells, cardiomyocytes have a limited capacity to proliferate as they are already terminally differentiated early after birth (Maillet et al., 2013; Paradis et al., 2014; Litviňuková et al., 2020).

Heart growth can be either physiological, occurring during development, pregnancy or in athletes as response to increased oxygen demand, or can be pathological eventually leading to HF when persisting. Physiological hypertrophy is mainly characterized by increased ventricular volume and a regulated increase in wall thickness. Unlike pathological hypertrophy, this mass increase does not involve structural abnormalities and is usually reversible (McMullen \& Jennings, 2007; Weeks \& McMullen, 2011; Maillet et al., 2013). Pathological hypertrophy leads to a reduced ventricular volume with an increase in wall thickness and is associated with alterations in calcium homeostasis, metabolism, as well as apoptosis and fibrosis. This process is mainly irreversible (Bentivegna et al., 1991; Ritchie \& Delbridge, 2006; McMullen \& Jennings, 2007; Dirkx et al., 2013; Nakamura \& Sadoshima, 2018). Prolonged pathological stimuli such as chronic hypertension, aortic stenosis, myocardial infarction or genetic cardiomyopathies can result in ventricular chamber dilatation leading to reduced contractile function and finally to transition to HF (Figure 1-2) (van Berlo et al., 2013; Tham et al., 2015; Nakamura \& Sadoshima, 2018). 


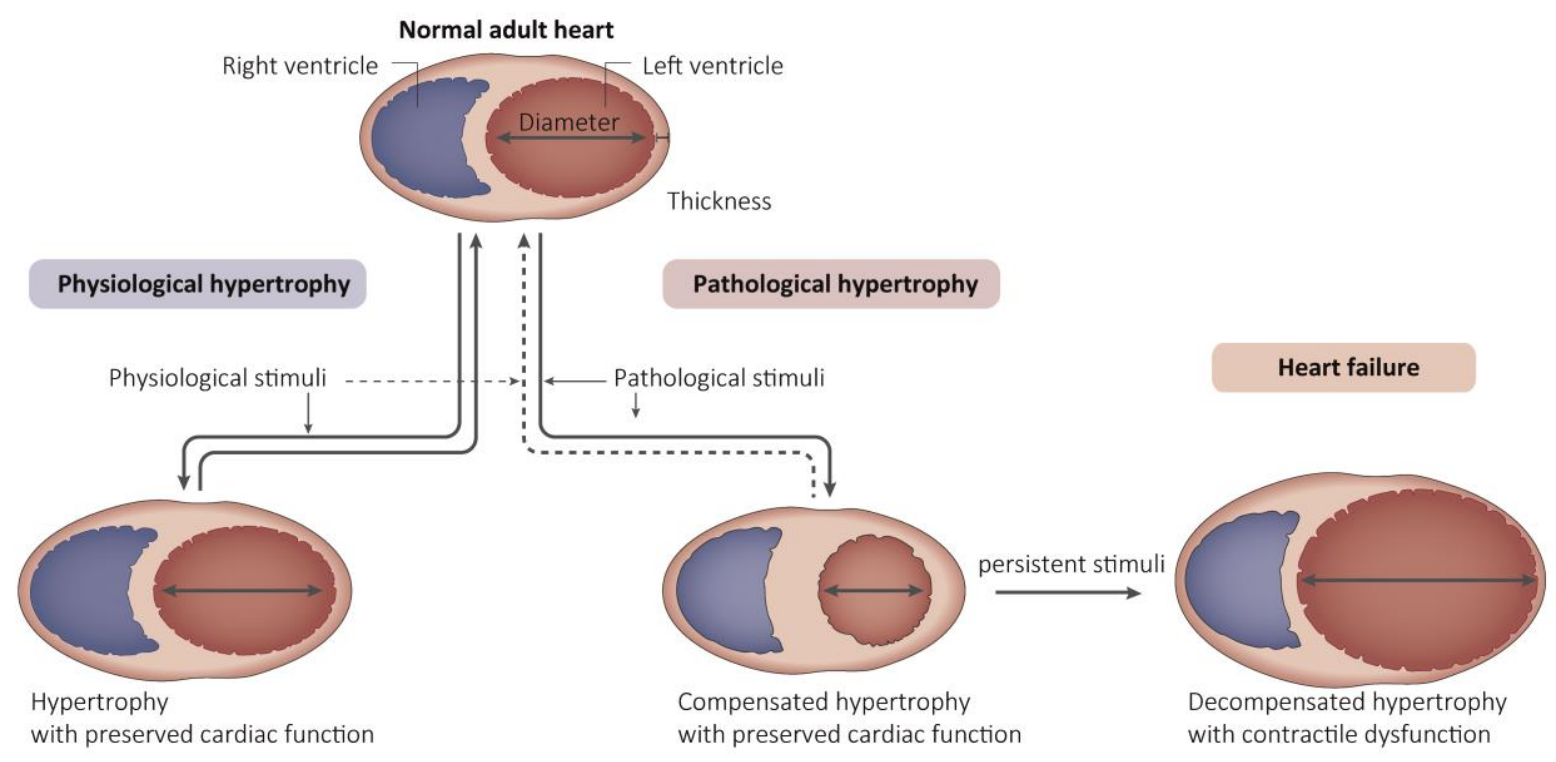

Figure 1-2 Overview of the transition from normal adult heart to hypertrophy and heart failure. The heart adapts to physiological or pathological stimuli by hypertrophy to preserve its cardiac function. Adaptive changes in physiological hypertrophy are characterized mainly by increased ventricular volume and are reversible if the stimuli relieve. In contrast, compensated hypertrophy due to pathological stimuli is characterized by reduced ventricular volume and increased wall thickness. If this stress persists, compensated hypertrophy can become decompensated with ventricular dilatation and loss of wall thickness and finally result in heart failure. Adapted and modified from (Nakamura \& Sadoshima, 2018).

\subsubsection{Molecular mechanisms of the transition from cardiac hypertrophy to heart failure}

The transition from cardiac hypertrophy to HF is characterized by several changes on molecular and cellular level in the myocardium (Figure 1-3). Cardiac hypertrophy becomes maladaptive when cell death, fibrosis, alterations in calcium cycling, fetal gene expression and energetic abnormalities occur together with myocardial growth (Oka \& Komuro, 2008; Nakamura \& Sadoshima, 2018). These events can be triggered by mechanical stress, hormones, cytokines and growth factors that act through various receptors on the cell membrane of cardiomyocytes (Heineke \& Molkentin, 2006; Wang et al., 2018a). The largest class of receptors among these are the G-protein coupled receptors (GPCRs) (Pierce et al., 2002). Neurohumoral and endocrine hormones such as angiotensin II, endothelin-1 and catecholamines bind to angiotensin II receptor, endothelin-1 receptor and $\alpha$ - adrenergic receptor or $\beta$ - adrenergic receptor, respectively (Heineke \& Molkentin, 2006; Salazar et al., 2007; Nakamura \& Sadoshima, 2018). Binding of catecholamines increases cAMP levels, leading to protein kinase A (PKA) activation. Angiotensin II and endothelin-1 binding results in activation of calcineurin and the calcium/calmodulin complex which then activates protein kinase $\mathrm{C} \alpha$ (PKC $\alpha)$ and calcium/calmodulin- dependent protein kinase type II (CaMKII). PKA, 
PKC $\alpha$ and CAMKII regulate cardiac contractility by calcium $\left(\mathrm{Ca}^{2+}\right)$ handling through phosphorylation of the cardiac ryanodine receptor 2 (RYR2) or phospholamban (PLN) that itself regulates sarcoplasmic reticulum $\mathrm{Ca}^{2+}$ ATPase (SERCA) activity (Salazar et al., 2007; van Berlo et al., 2013; Nakamura \& Sadoshima, 2018). Additionally, calcineurin activates nuclear factor of activated T-cells (NFAT) by dephosphorylation which leads to its translocation into the nucleus to initiate transcription of hypertrophic genes such as $\alpha$-actinin, brain natriuretic peptide (BNP) or $\beta$-myosin heavy chain (Molkentin et al., 1998; Heineke \& Molkentin, 2006; Stansfield et al., 2014). Another G-protein coupled pathway that promotes development of pathological hypertrophy is the mitogen-activated protein kinase (MAPK) signaling pathway. This signaling cascade is initiated by G-protein activation through binding of neurohumoral and endocrine hormones to their receptors resulting in activation of $\mathrm{p} 38$, the JUN N- terminal kinase (JNK) and the extracellular signal-regulated kinase (ERK) by the mitogen-activated protein kinase kinases 3/6 (MEK3/MEK6), mitogen-activated protein kinase kinases 4/7 (MEK4/MEK7) or mitogen-activated protein kinase kinases 1/2 (MEK1/MEK2), respectively. This activation leads to phosphorylation and therefore activation of myocyte enhancer factor2 (MEF2) by JNK, GATA binding protein 4 (GATA4) by p38 and NFAT by ERK and induce the transcription of pro-hypertrophic genes (Heineke \& Molkentin, 2006; Stansfield et al., 2014; Nakamura \& Sadoshima, 2018).

The natriuretic peptides atrial natriuretic peptide (ANP) and BNP show increased expression in hypertrophy as a compensatory mechanism. These peptides bind to their receptors and activate protein kinase G (PKG) which inhibits several hypertrophic pathways such as the calcineurin-NFAT pathway as described above. However, this antihypertrophic regulation is desensitized during the development of pathological hypertrophy (Nakamura \& Sadoshima, 2018).

Another important role during the transition to heart failure plays the cardiac metabolism. Alterations and dysregulation of the metabolism and the ATP production lead to the transition to pathological hypertrophy and HF by increased cardiomyocyte death and disturbed contractility (Neubauer, 2007; Doenst et al., 2013; Liew et al., 2017; Nakamura \& Sadoshima, 2018).

Apoptosis of cardiomyocytes can also be induced by several other pathways like Reactive Oxygen Species (ROS) triggered pathways (Harsdorf et al., 1999), angiotensin II pathway (Cigola et al., 1997; Kajstura et al., 1997; Gao et al., 2020), sympathetic stimulation (Adams et 
al., 1998; Singh et al., 2000) or cytokines triggered pathways (Arstall et al., 1999; Oka \& Komuro, 2008). In addition to apoptosis, excessive autophagy and necrosis also causes the loss of cardiomyocytes (Kostin et al., 2003; Nakayama et al., 2007; Oka \& Komuro, 2008). In summary, several different mechanisms are already identified to play a role in the transition to HF, but there is still a lack of knowledge about the precise mechanisms.

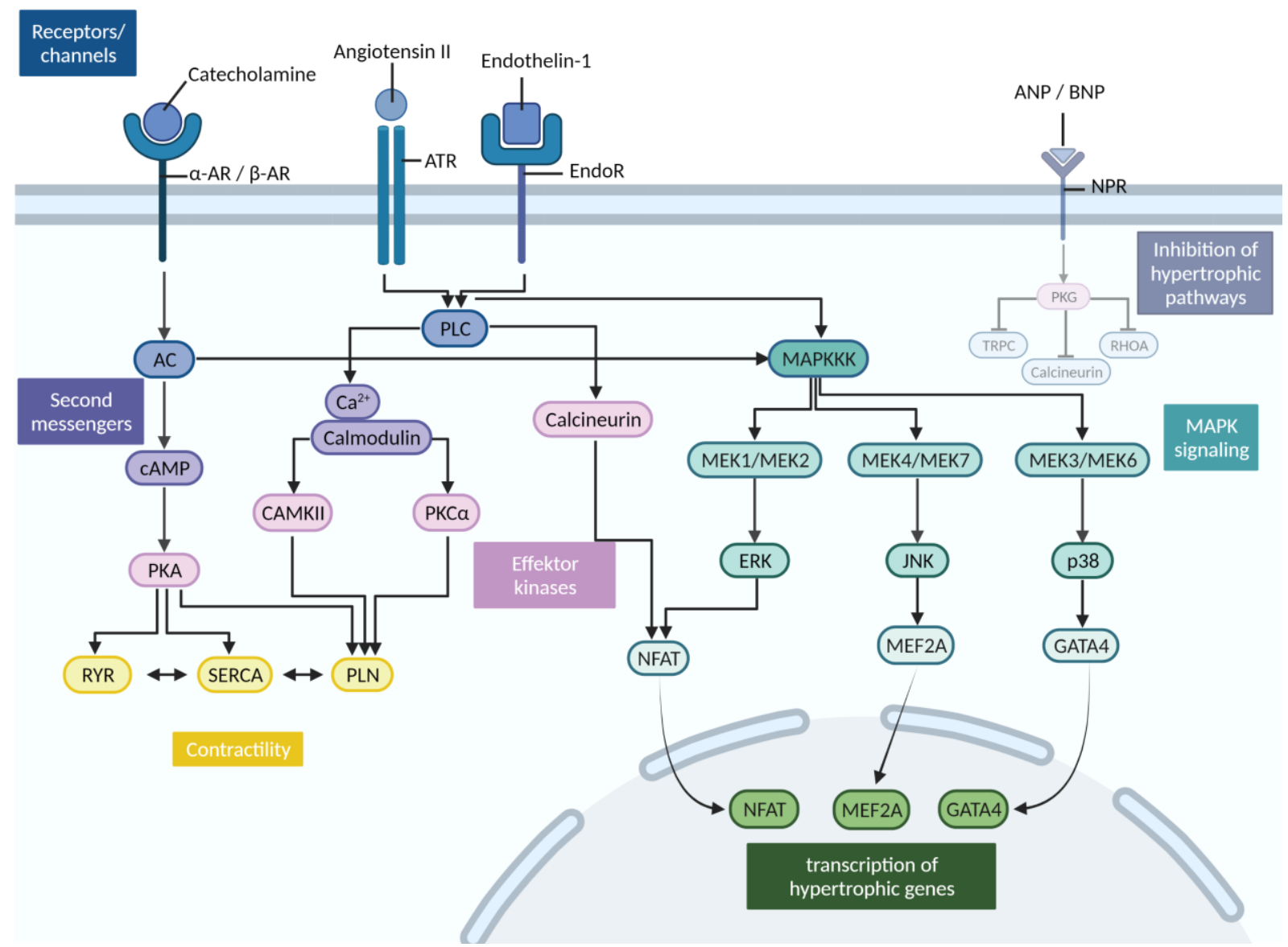

Figure 1-3 Scheme of selected signaling pathways involved in cardiac hypertrophy and the transition to heart failure. Neuroendocrine hormones initiate hypertrophic pathways leading to myocardial dysfunction. Natriuretic peptide-mediated activation of protein kinase G (PKG) leads to inhibition of hypertrophic pathways, although the natriuretic peptide receptor (NPR) is desensitized in hypertrophy and heart failure. $A C=$ adenylyl cyclase, ANP = atrial natriuretic peptide, $\alpha / \beta-A R=\alpha / \beta$-adrenergic receptor, $A T R=$ angiotensin-II receptor, $B N P=$ brain natriuretic peptide, $\mathrm{CaMKII}=$ calcium/calmodulin-dependent protein kinase type II, cAMP = 3'5'-cyclic AMP, EndoR = endothelin 1 receptor, ERK = extracellular regulated kinase, GATA4 = GATA binding protein 4, JNK = JUN $\mathrm{N}$-terminal kinase, $\mathrm{MAPKKK}=\mathrm{MAP}$ kinase kinase kinases, $\mathrm{MEF} 2 \mathrm{~A}=$ myocyte-specific enhancer factor $2 \mathrm{~A}, \mathrm{MEK}=$ mitogen-activated protein kinase kinase, NFAT = nuclear factor of activated T-cells, NPR = natriuretic peptide receptor, $\mathrm{p} 38=$ p38 kinases, $\mathrm{PKA} / \mathrm{C} \alpha / \mathrm{G}=$ protein kinase $\mathrm{A} / \mathrm{C} \alpha / \mathrm{G}, \mathrm{PLC}=$ phospholipase $\mathrm{C}, \mathrm{PLN}=$ phospholamban, $\mathrm{RHOA}=$ transforming protein RHOA, RYR = ryanodine receptor, SERCA = sarcoplasmic reticulum $\mathrm{Ca}^{2+} \mathrm{ATPase}$, TRPC = transient receptor protein channel. Figure modified from (van Berlo et al., 2013; Nakamura \& Sadoshima, 2018) and created with BioRender. 


\subsection{Calcium as a central regulator in the heart and its role in HF progression}

Calcium is a key player in the heart for excitation-contraction coupling. It is further involved in several signaling pathways regulating downstream effects. Dysregulation can lead to several heart diseases including heart failure (Beuckelmann et al., 1992; Frey et al., 2000; Heineke \& Molkentin, 2006).

Excitation-contraction coupling is initiated when an action potential reaches the myocyte along its transverse tubule (T-tubule) (Figure 1-4). The depolarization opens voltage-gated Ltype calcium channels (LTCC), resulting in calcium influx and triggers RYR2 to open by the calcium induced calcium release (CICR) process. The increased concentration of cytosolic calcium results in a conformational change of troponin, facilitating the formation of crossbridges between actin and myosin for myocardial contraction. Following the contraction, relaxation requires a rapid decrease of cytosolic calcium concentration. This is facilitated by SERCA and the Sodium Calcium Exchange (NCX) which pump calcium back into the sarcoplasmic reticulum (SR) or out of the cell, respectively (Luo \& Anderson, 2013; Eisner et al., 2017).

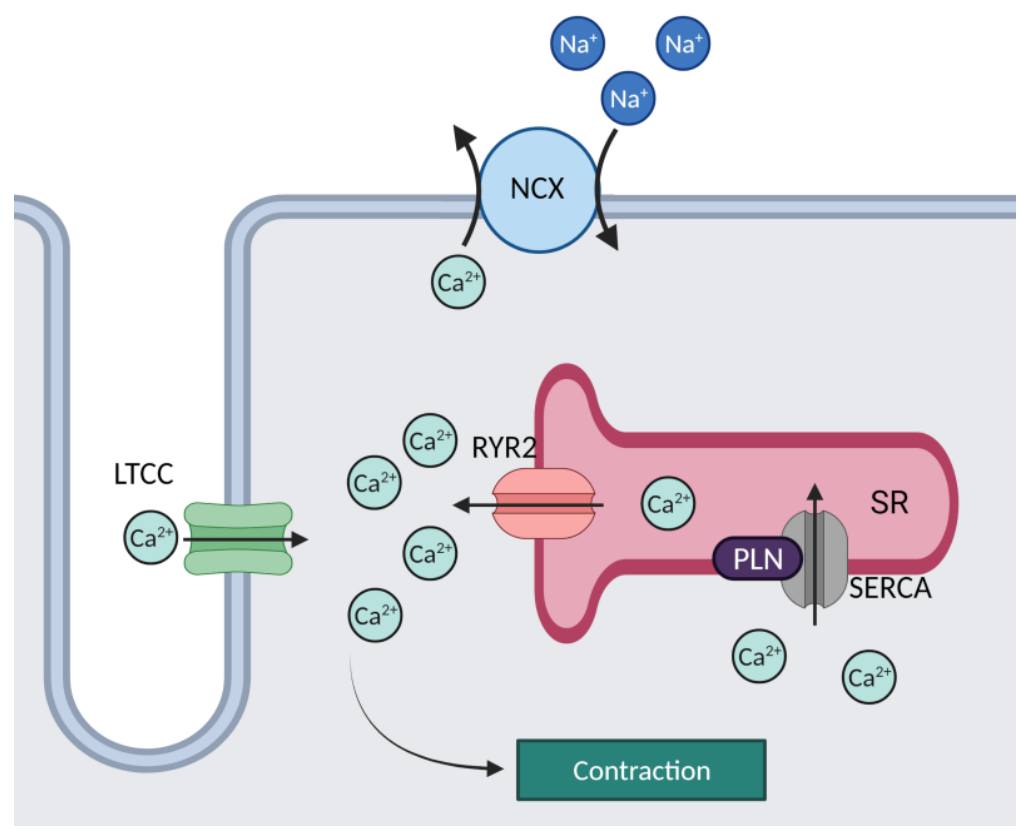

Figure 1-4 Schematic of cardiac excitation-contraction coupling. An action potential along the transverse tubules (T-tubule) initiates the opening of L-type $\mathrm{Ca}^{2+}$ channels (LTCC) enabling calcium $\left(\mathrm{Ca}^{2+}\right)$ to enter the cytosol and trigger $\mathrm{Ca}^{2+}$ release from the sarcoplasmic reticulum (SR) through the ryanodine receptor (RYR2). This intracellular calcium rise leads to contraction of the myofilaments in the cardiomyocytes. Relaxation follows by $\mathrm{Ca}^{2+}$ removal from the cytosol via the Sarcoplasmic/Endoplasmic Reticulum Calcium ATPase (SERCA) and the Sodium Calcium Exchange (NCX). Under resting conditions, SERCA is inhibited by binding of phospholamban (PLN). Figure adapted from (Shiels, 2011; Luo \& Anderson, 2013) and created with BioRender. 
Heart failure can be associated with dysregulation in excitation-contraction coupling characterized by decreased calcium transients, slower transient decay and reduced SR calcium load due to an enhanced calcium leak and reduced calcium uptake into the SR. All these events can impair myocyte contractility and relaxation. These abnormalities are a result of changes in various calcium handling proteins or defects in the T-tubules and play an important role in the development of HF (Piacentino et al., 2003; Wei et al., 2010; Luo \& Anderson, 2013). One of these proteins is the ion channel RYR2. RYR2 can be modified by PKA, CAMKII, protein phosphatase 1 and 2A, calmodulin and 12.6 kDa FK506-binding protein (FKBP12.6). PKA as well as CAMKII can phosphorylate RYR2 and therefore increase its opening probability, resulting in arrhythmias and myocardial dysfunction. Hyperphosphorylation by PKA can even lead to SR Ca ${ }^{2+}$ leak in HF (Ai et al., 2005; Sag et al., 2009; Luo \& Anderson, 2013). LTCC activity is regulated by CAMKII, PKA and $\beta$-adrenergic stimulators and upregulation can result in lasting channel openings as well as increased opening probability which both rise intracellular calcium levels and therefore promote HF and arrhythmias (Reuter, 1983; Wu et al., 1999; Bers \& Morotti, 2014).

Important for the calcium homeostasis and therefore key players in hypertrophy and HF are the two pumps NCX and SERCA. In the inactive state, SERCA is inhibited by dephosphorylated PLN (Akin et al., 2013). Phosphorylation of PLN by PKA reduces the association between SERCA and PLN, leading to an increased calcium uptake into the SR (Kranias \& Hajjar, 2012). In heart failure, SERCA activity was shown to be decreased leading to a slower systolic calcium transient decay and a decreased amplitude. As a consequence, relaxation and contraction are impaired (Mercadier et al., 1990; Lipskaia et al., 2007; Eisner et al., 2013). To test if the contractile function can be restored, SERCA was overexpressed in isolated cardiomyocytes from failing human hearts and indeed could normalize calcium handling and contractility (Del Monte et al., 1999).

SR calcium load via SERCA is also responsible to sensitize RYR2. As a consequence, an increased cytoplasmic and SR calcium load can induce abnormal calcium release events which activate NCX and therefore produce aftercontractions and afterdepolarizations resulting in arrhythmias (Venetucci et al., 2008).

Impaired calcium homeostasis in cardiomyocytes is a major factor during progression to HF. It is crucial for normal cardiac contractile function and dysregulation due to transcriptional or 
posttranscriptional modifications of calcium channels impairs normal cardiac function. Therefore, calcium as a key regulator is an important target for therapeutic strategies to maintain calcium homeostasis and contractile function and further prevent development of cardiac remodeling and HF.

\subsection{Transcriptomics of human and mouse during the transition to heart}

\section{failure}

To identify novel targets in the transition to heart failure in response to pressure overload (PO), previous studies in the group performed a deep RNA-sequencing screen of human myocardium of aortic stenosis patients and compared these data to control myocardium from potential organ donors. These samples were obtained from aortic valve replacement surgeries. In total, three groups were compared: non-failing control (NF) containing four biopsy samples, compensated hypertrophy $(\mathrm{CH})$ and moderate heart failure (HF), both containing five samples each. Differences between the two pathological groups are due to the ejection fraction (EF) with either being higher than $55 \%$ for the $\mathrm{CH}$ group, or with values around $33 \%$ for the HF group. To further identify conserved candidate genes, similar stages of PO-induced hypertrophy and heart failure in the transverse aortic constriction (TAC) mouse model were RNA-screened and compared to the human biopsy samples. $\mathrm{CH}$ samples were compared to the mouse model one week after TAC with a preserved EF of about $63 \%$, whereas HF samples were compared to mouse samples obtained 8 weeks post TAC having a reduced $\mathrm{EF}$ of about $47 \%$. The comparison of these two datasets identified 25 genes that are regulated similarly in human and mouse heart during disease progression (Figure 1-5A). Among these genes, CRISPLD1 was identified as a conserved candidate gene as its expression levels are low in non-failing myocardium of human and mice but increased in compensated hypertrophy (log2foldchange $=1.6)$ and 1 week post-transverse aortic constriction (1WTAC) (log2foldchange $=1.5$ ). These levels increased even more in heart failure (log2foldchange $=2.6)$ and 8 weeks post-transverse aortic constriction (8WTAC) (log2foldchange $=2.3$ ) (Figure 1-5B). This data was finally validated by RT-qPCR (Figure 1-5C) (Khadjeh et al., 2020). 
A

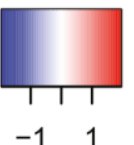

Row Z-Score
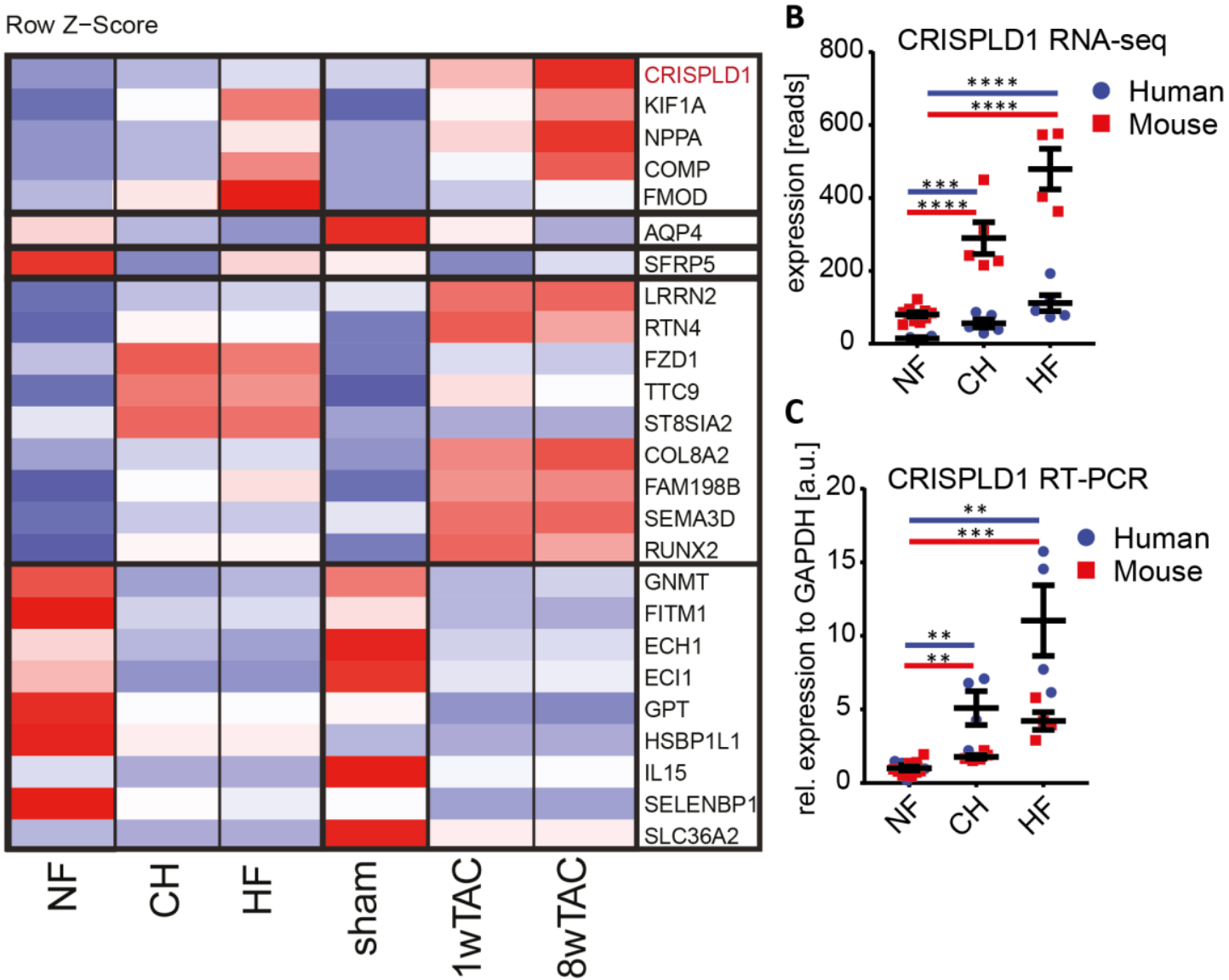

Figure 1-5 Transcriptomics of PO-induced $\mathrm{CH}$ and $\mathrm{HF}$ in human and mouse myocardium. Heatmap of conserved differentially expressed genes in human and mouse during the transition to HF. The logFC of median normalized counts are represented by the light blue to dark red shading scale. Genes that show similar expression during disease progression in mouse and human are framed. CRISPLD1 is highlighted in red. (B) RNA-sequencing expression profile of CRISPLD1 in NF, CH and HF (sham $=\mathrm{NF}, 1 \mathrm{WTAC}=\mathrm{CH}$ and $8 \mathrm{WTAC}=\mathrm{HF}$ ). Shown are the reads (rpkm) of aortic stenosis patients and mice. P-values are FDR-corrected. (C) Validation of transcriptomics by RTqPCR of human and mouse $\mathrm{NF}, \mathrm{CH}$ and $\mathrm{HF}$ myocardium. a.u. = arbitrary units, $\mathrm{CH}=$ compensated hypertrophy, $\mathrm{EF}$ $=$ ejection fraction, $\mathrm{HF}=$ moderate heart failure, $\mathrm{NF}=$ non-failing control, rel. $=$ relative, $1 \mathrm{WTAC}=1$ week post transverse aortic constriction, $8 \mathrm{WTAC}=8$ weeks post-transverse aortic constriction. Error bars represent mean \pm SEM, ${ }^{* *}=p$ value $\leq 0.01,{ }^{* * *}=p$ value $\leq 0.001,{ }^{* * *}=p$ value $\leq 0.0001, n=4-5 /$ group. Adapted and modified from (Khadjeh et al., 2020).

\subsection{Cysteine-rich secretory protein LCCL domain-containing 1 (CRISPLD1)}

Cysteine-rich secretory protein LCCL domain-containing 1 (CRISPLD1) encodes a protein with a total length of 500 amino acids (aa) which belongs to the cysteine-rich secretory proteins, Antigen 5 , and pathogenesis-related 1 protein (CAP) superfamily and additionally contains two limulus factor C, Coch-5b2 and LgI1 (LCCL) domains and two allergen V5/Tpx-1 conserved sites (Figure 1-6A). The protein is predicted to be secreted due to a signaling peptide and its homology to the CAP superfamily proteins. However, its function is completely undescribed 
in literature.

CAP superfamily proteins are usually secreted and have an either autocrine or paracrine function, broadly regulating signaling pathways. Due to their structural conservation all members of this superfamily show fundamentally similar functions (Gibbs et al., 2008). Few members were already reported to be related to heart diseases. For example, peptidase inhibitor 16 (PI16), one member of the CAP superfamily, was described as a negative regulator of cardiomyocyte size in mice being highly upregulated in the HF mouse model and human heart failure (Frost \& Engelhardt, 2007). Another subfamily of the CAPs are cysteine-rich secretory proteins (CRISPS) with the unique feature of having ion channel regulator activity and with being highly expressed and conserved in the male reproductive tract, as well as in the venom of poisonous reptiles (Gibbs et al., 2006; Gibbs et al., 2008). One example is the toxin helothermine, a CRISP protein from the venom of the Mexican beaded lizard Heloderma horridum horridum. Helothermine blocks potassium channels, the cardiac ryanodine receptor and SR Ca ${ }^{2+}$ release (Nobile et al., 1994; Morrissette et al., 1995; Nobile et al., 1996). The allergen V5/Tpx-1 site of CRISPLD1 itself shows a high level of sequence homology to CRISP1 and CRISP2 signatures which are also part of helothermine (PROSITE patterns PS01009 and PS01010) (Figure 1-6B).

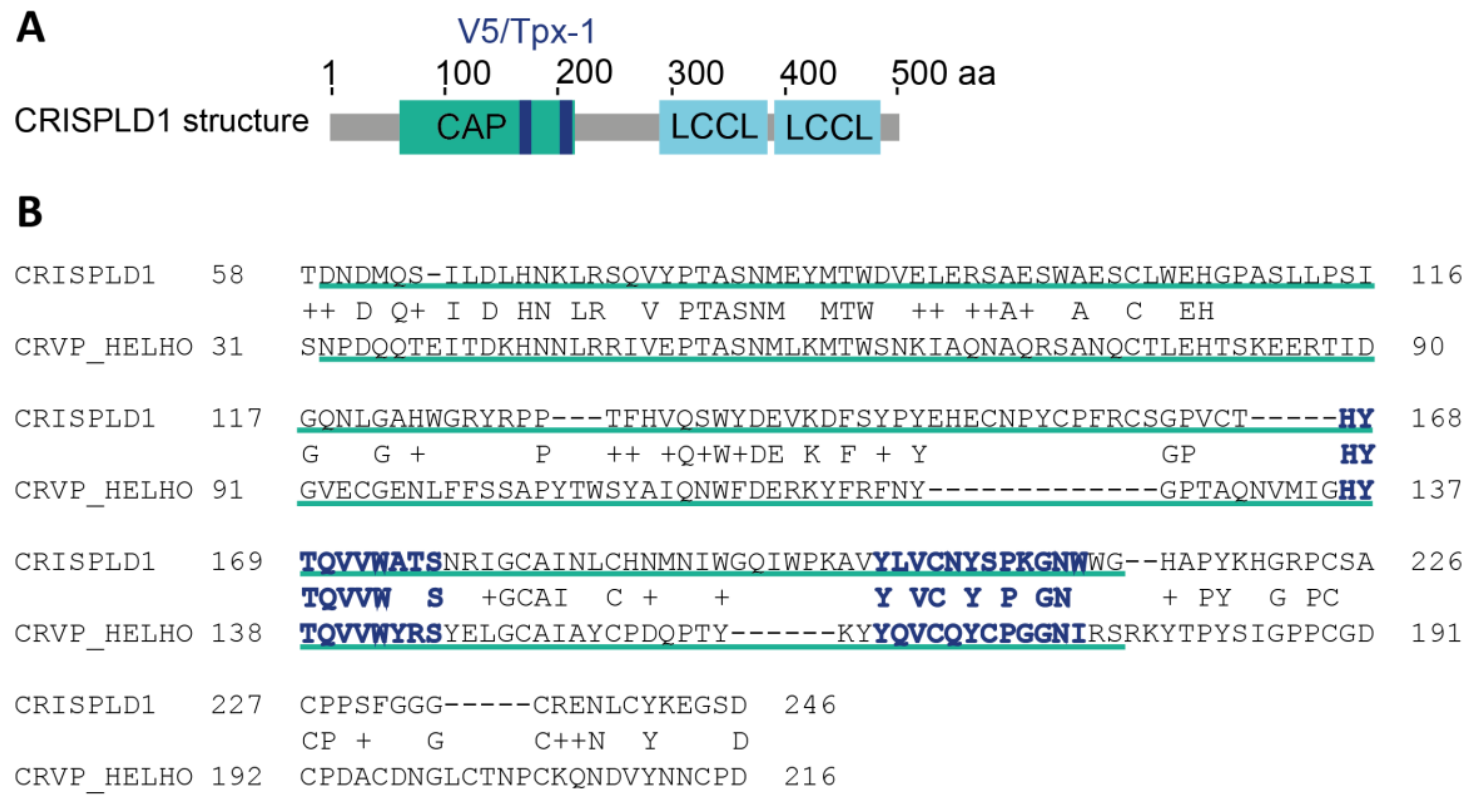

Figure 1-6 Protein structure of CRISPLD1 and alignment to helothermine. (A) CRISPLD1 with a total length of 500 amino acids (aa) contains a CAP domain (155 aa, highlighted in turquoise), two V5/Tpx-1 conserved sites (10 aa and 12 aa, marked in dark blue) and two LCCL domains (highlighted in light blue). (B) Alignment of CRISPLD1 to helothermine shows conservation in the CAP domain (underlined in turquoise) and the two V5/Tpx-1 related sites (letters highlighted in red, CRISP1 and CRISP2, respectively). Alignment was generated using blastp. CAP = CRISP/Antigen 5/PR-1, CRISPLD1 = Cysteine-rich secretory protein LCCL domain-containing 1, HELHO = 
helothermine, $\mathrm{LCCL}=$ Limulus factor C, Coch-5b2 and Lgl1, Tpx-1 = testis-specific protein 1. Adapted and modified from (Khadjeh et al., 2020).

CRISPLD1 was previously found in urinary exosomes from prostatic secretions (Principe et al., 2013) and human parotid glands secret (Gonzalez-Begne et al., 2009). It is suggested to play a role in the folic acid pathway (Chiquet et al., 2011) and progesterone resistance in endometrial cancer (Li et al., 2019) and shows altered methylation during male infertility (Sujit et al., 2018). Furthermore, it was detected, among others, as a regulator of hematopoietic stem and progenitor cell repopulation (Holmfeldt et al., 2016) and found to be highly downregulated in

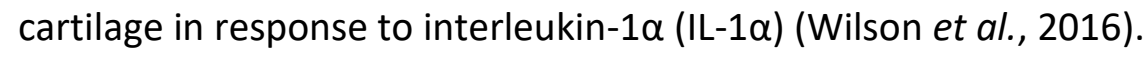

CRISPLD1 was only recently identified as a key gene in human platelets in acute myocardial infarction (AMI) patients (Zhang et al., 2021) and CRISPLD1 polymorphism was found to regulate antiplatelet effects of clopidogrel in Han Chinese patients with coronary heart disease (Wang et al., 2018b). Except for these two publications associating CRISPLD1 with heart diseases, its function in the heart is not further described in literature.

\subsection{Aims of the study}

With the focus on identifying novel targets in the transition to heart failure, transcriptomics of human and mouse PO diseased hearts were preliminary performed in the group. Analysis identified 25 candidate genes being differentially regulated during the transition to heart failure but showing conserved expression pattern in human and mouse. Among these genes, CRISPLD1 was identified as a candidate gene to study as it showed increased expression during disease development and its function in the heart is uncharacterized. Additionally, protein domain analysis showed that the protein contains (besides a CAP and two LCCL domains) an allergen V5/Tpx-1 related, conserved sites. These are known to be evolutionary related to the toxin helothermine found in the Mexican beaded lizard (Heloderma horridum horridum) and known to regulate ion channels, block the cardiac ryanodine receptor and calcium induced calcium release.

In order to elucidate the function of CRISPLD1 in the heart, especially identify its role during the transition to heart failure, I investigated CRISPLD1 function in the mouse as well as in induced pluripotent stem cell derived cardiomyocytes (iPSC-CMs). Therefore, the aims of my thesis are split in two parts: 
1) Characterization of CRISPLD1 in the iPSC-CM model

- Generation and validation of CRISPLD1 knockout (KO) in IPSC-CMs

To investigate the effects of CRISPLD1-deficient iPSC-CMs, I will generate a CRISPLD1KO line. Exon 7 will be targeted using CRISPR/Cas9. The underlying wildtype (WT) cell line will serve as an isogenic control. Validation of CRISPLD1-KO will be done by DNA and mRNA expression analysis.

- Influence of CRISPLD1 KO on cardiomyocytes

I will examine the effects of CRISPLD1-KO in iPSC-CMs by immunofluorescence and RT-qPCR of several cardiomyocyte specific expression markers.

- Characterization of calcium homeostasis in CRISPLD1-deficient iPSC-CMs Changes in the calcium homeostasis of CRISPLD1-deficient iPSC-CMs will be observed by confocal calcium imaging.

- Examination of the influence of helothermine treatment on the calcium homeostasis in WT iPSC-CMs and CRISPLD1-deficient iPSC-CMs

To elucidate any similarity to helothermine, I will treat WT and CRISPLD1-deficient iPSC-CMs with the toxin and compare them. I will further examine the influences of helothermine on the calcium homeostasis.

- Analysis of downstream pathways and networks after CRISPLD1 KO

To get an idea of downstream pathways targeted by CRISPLD1, I will perform transcriptomics and proteomics of CRISPLD1 KO iPSC-CMs.

\section{2) Characterization of Crispld1 in the mouse model}

- Generation and validation of cardiomyocyte-specific CRISPLD1 knockout in mice To enable in vivo analysis of Crispld1 loss-of-function specifically in the heart, I will generate conditional Crispld1 KO mice by Cre/loxP recombination using the Myh6-Cre mouse line. The conditional Crispld1 mouse strain was previously ordered from KOMP repository and flanks exon 5 of Crispld1. The success of recombination to generate cardiomyocyte-specific deletion will be analyzed on DNA level and by mRNA expression.

- Generation and validation of global Crispld1 knockout in mice In order to elucidate Crispld1 function during development, I will generate mice with ubiquitous Crispld1 deletion by Cre/loxP recombination using the previously ordered 
conditional Crispld1 mouse strain. Deletion of Crispld1 will be validated by DNA and mRNA expression.

- Examination of the impact of Crispld1 knockout on the survival of mice

To investigate if Crispld1 influences mortality, I will monitor the survival of Crispld1 $\mathrm{KO}$ and control mouse lines for one year after birth and investigate any differences between a CM-specific and a global Crispld1 KO.

- Analysis of the influence of Crispld1 KO on heart function and morphology in the Crispld1 KO mouse lines

In order to characterize Crispld1 functionally in the heart, I will analyze the heart phenotype of KO mice by histology, echocardiography and cardiomyocyte morphometry.

- Characterization of calcium homeostasis in isolated mouse cardiomyocytes to compare to CRISPLD1-deficient iPSC-CMs

To compare the human iPSC-CMs to the mouse model, I will isolate mouse cardiomyocytes to measure influences of Crispld1 $\mathrm{KO}$ on the calcium homeostasis. Afterwards, I will compare these findings to the results from confocal calcium imaging of iPSC-CMs. 


\section{Materials and Methods}

\subsection{Animals}

\subsubsection{C57BL/6 mice}

C57BL/6 mice were obtained from Charles River or bred in the animal facility of the university medical center Göttingen.

\subsubsection{C57BL/6N-Tg(Cag-Flpo)1Afst/Mmcd (CAG-FlpO) mice}

CAG-FIpO mice were a kind gift from Prof. Dr. Elisabeth Zeisberg and used to delete the FRT sites in Crispld ${ }^{1 \mathrm{tm} 1 \mathrm{a}(\mathrm{KOMP}) W t s i}$ mice to convert to a conditional allele. CAG-flpO mice were originally generated by Prof. Anastassiadis (Kranz et al., 2010).

\subsubsection{B6N.FVB(B6)-Tg(Myh6-cre)2182Mds/J (Myh6-Cre) mice}

To generate CM-specific Crispld1 KO mice, Myh6-Cre mice were used and were a kind gift from Prof. Karl Toischer and originally generated by Prof. Michael Schneider (Agah et al., 1997).

\subsubsection{B6: $\mathrm{Tg}(\mathrm{CMV}-\mathrm{Cre})$ (CMV-Cre) mice}

The CMV-Cre mouse line used for the generation of the ubiquitous deletion of Crispld1 was a kind gift from Dr. Muriel Lizé and originally generated by Prof. Klaus Rajewsky (Schwenk et al., 1995).

\subsubsection{Crispld1 ${ }^{\text {tm1a(KOMP)Wtsi }}$ mice}

The Crispld 1 mouse strain used to generate Crispld1 knockout mice was created from ES cell clone EPD0205_4_D12 generated by the Wellcome Trust Sanger Institute and made into mice by the KOMP Repository (www.komp.org) and the Mouse Biology Program (www.mousebiology.org) at the University of California Davis. Methods used to create the CSD targeted alleles have been published (Skarnes et al., 2011). 


\subsection{Cell lines}

Table 1: Cell lines

\begin{tabular}{|l|l|l|l|l|}
\hline \multicolumn{1}{|c|}{ Name } & \multicolumn{1}{|c|}{ Number } & \multicolumn{1}{|c|}{ origin } & \multicolumn{1}{c|}{$\begin{array}{c}\text { Reprogramming } \\
\text { system }\end{array}$} & \multicolumn{1}{c|}{ publication } \\
\hline ipWT1.3 & GOEi014-B.3 & Dermal fibroblast & $\begin{array}{l}\text { 4-in-1 CoMiP } \\
\text { reprogramming } \\
\text { plasmid, feeder- } \\
\text { free culture } \\
\text { conditions }\end{array}$ & $\begin{array}{l}\text { (El-Battrawy et } \\
\text { al., 2018) }\end{array}$ \\
\hline HEK293T & - & $\begin{array}{l}\text { human } \\
\text { embryonic } \\
\text { kidney cells }\end{array}$ & - & $\begin{array}{l}\text { (DuBridge } \text { et al., } \\
\text { 1987) }\end{array}$ \\
\hline iWT.D2.1 & $\begin{array}{l}\text { GOED001-A.1; } \\
\text { FB2-iPS1 }\end{array}$ & Dermal fibroblast & $\begin{array}{l}\text { STEMCCA } \\
\text { lentivirus }\end{array}$ & $\begin{array}{l}\text { (Cyganek et al., } \\
\text { 2018) }\end{array}$ \\
\hline
\end{tabular}

\subsection{Cell culture media and solutions}

Table 2: Media, supplements, and solutions for cell culture

\begin{tabular}{|l|l|l|}
\hline \multicolumn{1}{|c|}{ Product } & \multicolumn{1}{|c|}{ Manufacturer } & \multicolumn{1}{c|}{ Catalogue number } \\
\hline B-27 Supplement (50x) & Thermo Fisher Scientific & 17504044 \\
\hline CHIR99021 & Millipore & 361571 \\
\hline Dimethyl sulfoxide (DMSO) & Sigma Aldrich & D2650 \\
\hline $\begin{array}{l}\text { DMEM, high glucose, no } \\
\text { glutamine }\end{array}$ & Thermo Fisher Scientific & 11960044 \\
\hline $\begin{array}{l}\text { Dulbecco's phosphate- } \\
\text { buffered saline (DPBS) }\end{array}$ & Thermo Fisher Scientific & 14190094 \\
\hline Fetal Bovine Serum (FBS) & Thermo Fisher Scientific & 10270106 \\
\hline HEPES solution & Sigma Aldrich & H0887 \\
\hline Human recombinant Albumin & Sigma Aldrich & A9731 \\
\hline IWP-2 & Millipore & 681671 \\
\hline L-ascorbic acid 2-phosphate & Sigma Aldrich & A8960 \\
\hline L-Glutamine (200 mM) & Thermo Fisher Scientific & 25030024 \\
\hline $\begin{array}{l}\text { Lipofectamine 3000 } \\
\text { Transfection Reagent }\end{array}$ & Thermo Fisher Scientific & L3000001 \\
\hline Matrigel & Th. Geyer & 7611631 \\
\hline $\begin{array}{l}\text { MEM Non-Essential Amino } \\
\text { Acids Solution (NEAA) }\end{array}$ & Thermo Fisher Scientific & 11140035 \\
\hline
\end{tabular}




\begin{tabular}{|l|l|l|}
\hline $\begin{array}{l}\text { Opti-MEM I Reduced Serum } \\
\text { Medium }\end{array}$ & Thermo Fisher Scientific & 31985062 \\
\hline $\begin{array}{l}\text { Penicillin-Streptomycin } \\
\text { solution }\end{array}$ & Th. Geyer & P4333 \\
\hline $\begin{array}{l}\text { RPMI } 1640 \text { Medium, GlutaMAX } \\
\text { Supplement, HEPES }\end{array}$ & Thermo Fisher Scientific & 72400021 \\
\hline $\begin{array}{l}\text { RPMI 1640 Medium, no } \\
\text { glucose }\end{array}$ & Thermo Fisher Scientific & 11879020 \\
\hline Sodium DL-lactate solution & Sigma Aldrich & L4263 \\
\hline StemFlex Medium & Thermo Fisher Scientific & A3349401 \\
\hline Thiazovivin (TZV) & Sigma Aldrich & $420220-\mathrm{M}$ \\
\hline Trypsin-EDTA (0.25\%) & Thermo Fisher Scientific & 25200056 \\
\hline Versene solution & Thermo Fisher Scientific & 15040066 \\
\hline
\end{tabular}

Table 3: Human cell culture medium and solution components

\begin{tabular}{|c|c|}
\hline Medium / solution & Components \\
\hline Cardio Cryopreservation Medium & $\begin{array}{l}90 \% \text { FBS } \\
10 \% \text { DMSO } \\
2 \mu \mathrm{M} \mathrm{TZV}\end{array}$ \\
\hline Cardio Culture Medium & $\begin{array}{l}500 \mathrm{ml} \text { RPMI } 1640 \text { Medium, GlutaMAX } \\
\text { Supplement, HEPES } \\
10 \mathrm{ml} \mathrm{B27} \mathrm{supplement}\end{array}$ \\
\hline Cardio Differentiation Medium & $\begin{array}{l}500 \mathrm{ml} \text { RPMI } 1640 \text { Medium, GlutaMAX } \\
\text { Supplement, HEPES } \\
0.5 \mathrm{mg} \text { human recombinant albumin } \\
0.2 \mathrm{mg} / \mathrm{ml} \mathrm{L} \text {-ascorbic acid 2-phosohate }\end{array}$ \\
\hline Cardio Digestion Medium & $\begin{array}{l}40 \mathrm{ml} \text { Cardio Culture Medium } \\
10 \mathrm{ml} \mathrm{FBS} \text { (heat-inactivated) } \\
500 \mu \mathrm{l} \text { Penicillin - Streptomycin } \\
50 \mu \mathrm{l} \mathrm{TZV}\end{array}$ \\
\hline Cardio Selection Medium & $\begin{array}{l}500 \mathrm{ml} \text { RPMI } 1640 \text { without Glucose and HEPES } \\
2 \mathrm{ml} \text { Lactate / HEPES (1M Stock solution) } \\
250 \mathrm{mg} \text { human albumin } \\
100 \mathrm{mg} \text { L-Ascorbic Acid 2-Phosphate }\end{array}$ \\
\hline CHIR (4 mM) & $5 \mathrm{mg}$ CHIR99021 dissolved in $894 \mu \mathrm{l}$ DMSO \\
\hline HEK medium & $\begin{array}{l}450 \mathrm{ml} \text { DMEM } \\
50 \mathrm{ml} \mathrm{FBS} \mathrm{(heat-inactivated)} \\
5 \mathrm{ml} \text { NEAA } \\
5 \mathrm{ml} \text { L-Glutamine }\end{array}$ \\
\hline
\end{tabular}




\begin{tabular}{|l|l|}
\hline IWP2 (5 mM) & $\begin{array}{l}10 \mathrm{mg} \text { dissolved in } 4.28 \mathrm{mg} \text { DMSO and } \\
\text { incubated for } 10 \mathrm{~min} \text { at } 37^{\circ} \mathrm{C}\end{array}$ \\
\hline Lactate / HEPES (1 M) & $\begin{array}{l}3 \mathrm{ml} \text { sodium DL-lactate dissolved in } 18 \mathrm{ml} 1 \mathrm{M} \\
\text { HEPES solution }\end{array}$ \\
\hline Matrigel (1:60 dilution) & $\begin{array}{l}500 \mu l \text { Matrigel } \\
29.5 \mu l \text { PBS }\end{array}$ \\
\hline TZV (2 mM) & $10 \mathrm{mg}$ TZV dissolved in $16.05 \mathrm{ml}$ DMSO \\
\hline
\end{tabular}

\subsection{Chemicals}

Table 4: Chemicals

\begin{tabular}{|c|c|c|}
\hline Product & Manufacturer & Catalogue number \\
\hline $\begin{array}{l}\text { 2,3-Butanedione Monoxime } \\
\text { (BDM) }\end{array}$ & Sigma Aldrich & B0753 \\
\hline $\begin{array}{l}\text { 4-(2-hydroxyethyl)-1- } \\
\text { piperazineethanesulfonic acid } \\
\text { (HEPES) }\end{array}$ & Roth & HN77.1 \\
\hline 4x Laemmli Sample Buffer & Bio-Rad & 1610747 \\
\hline $\begin{array}{l}\text { Alt-R S.p. HiFi Cas9 Nuclease } \\
\text { 3NLS }\end{array}$ & IDT DNA Technologies & 1081061 \\
\hline Alt-R CRISPR-Cas9 tracrRNA & IDT DNA Technologies & 1072534 \\
\hline Ammonium Persulfate (APS) & Bio-Rad & 1610700 \\
\hline Boric Acid & Th. Geyer & 15663 \\
\hline Bovine calf serum (BCS) & Sigma Aldrich & $12133 c$ \\
\hline Bovine Serum Albumin (BSA) & Sigma Aldrich & A9647 \\
\hline $\begin{array}{l}\text { Calcium Chloride solution } \\
\left(\mathrm{CaCl}_{2}\right)\end{array}$ & Sigma Aldrich & 21115 \\
\hline DirectPCR tail lysis reagent & Viagen Biotech & B102-T \\
\hline $\begin{array}{l}\text { di-Sodium hydrogen } \\
\text { phosphate dihydrate }\left(\mathrm{Na}_{2} \mathrm{HPO}_{4}\right. \\
\left.\times 2 \mathrm{H}_{2} \mathrm{O}\right)\end{array}$ & Th Geyer & 4984.2 \\
\hline Dithiothreitol (DTT) & Thermo Fisher Scientific & R0861 \\
\hline DNA Gel Loading Dye (6X) & Thermo Fisher Scientific & R0611 \\
\hline dNTP Mix & Bioline & BIO-39029 \\
\hline DPX Mounting Media & Fisher Scientific & 15538321 \\
\hline Ethanol & Merck & 1009831000 \\
\hline $\begin{array}{l}\text { Ethylenediaminetetraacetic } \\
\text { acid (EDTA) }\end{array}$ & Roth & 8040.3 \\
\hline
\end{tabular}




\begin{tabular}{|c|c|c|}
\hline Fast Green FCF & Sigma Aldrich & F7252 \\
\hline Fluo-4, AM & Thermo Fisher Scientific & F14201 \\
\hline Fluoromount-G & Thermo Fisher Scientific & 00-4958-02 \\
\hline $\begin{array}{l}\text { Gene Ruler 100bp plus DNA } \\
\text { Ladder }\end{array}$ & Thermo Fisher Scientific & SM0322 \\
\hline Glucose & Roth & HN06.2 \\
\hline Glycine & Roth & 3908.3 \\
\hline GoTaq DNA Polymerase & Promega & M3001 \\
\hline HBSS without Phenol Red & Gibco & 14025092 \\
\hline Helothermine & Venomtech & - \\
\hline Hydrochloric acid (HCl) & Roth & K025.1 \\
\hline Forene $100 \%$ & Abbvie & - \\
\hline Fura-2 AM & Thermo Fisher Scientific & F1221 \\
\hline Isoprenaline & Sigma Aldrich & 15627 \\
\hline Isopropanol & Th. Geyer & 1.09634 .1000 \\
\hline Liberase TM & Sigma Aldrich & 5401119001 \\
\hline Magnesium chloride $\left(\mathrm{MgCl}_{2}\right)$ & Roth & KK36.2 \\
\hline $\begin{array}{l}\text { Magnesium sulphate } \\
\text { heptahydrate }\left(\mathrm{MgSO}_{4} \times 7 \mathrm{H}_{2} \mathrm{O}\right)\end{array}$ & Roth & T888.1 \\
\hline MangoTaq Polymerase & Bioline & BIO-21082 \\
\hline Midori Green & Biozym & 617004 \\
\hline Milk powder & Th. Geyer & $\mathrm{t} 145.1$ \\
\hline Oligo(dT)20 Primer & Thermo Fisher Scientific & 18418020 \\
\hline peqGold Universal Agarose & VWR & $35-1020$ \\
\hline $\begin{array}{l}\text { Phusion Green Hot Start II } \\
\text { High-Fidelity DNA Polymerase }\end{array}$ & Thermo Fisher Scientific & F537S \\
\hline Picric acid solution & Sigma Aldrich & P6744 \\
\hline $\begin{array}{l}\text { Pierce Protease and } \\
\text { Phosphatase Inhibitor Mini } \\
\text { Tablets }\end{array}$ & Thermo Fisher Scientific & A32961 \\
\hline $\begin{array}{l}\text { Pierce Protease Inhibitor Mini } \\
\text { Tablets }\end{array}$ & Thermo Fisher Scientific & A32955 \\
\hline Pluoronic F-127 & Thermo Fisher Scientific & P3000MP \\
\hline Ponceau S solution & Sigma Aldrich & P7170 \\
\hline Potassium Chloride $(\mathrm{KCl})$ & Roth & HNO2.1 \\
\hline $\begin{array}{l}\text { Potassium dihydrogen } \\
\text { phosphate }\left(\mathrm{KH}_{2} \mathrm{PO}_{4}\right)\end{array}$ & Roth & 3904.2 \\
\hline
\end{tabular}




\begin{tabular}{|c|c|c|}
\hline $\begin{array}{l}\text { Potassium hydrogen carbonate } \\
\left(\mathrm{KHCO}_{3}\right)\end{array}$ & Roth & P748.1 \\
\hline $\begin{array}{l}\text { Precision Plus Protein }{ }^{\mathrm{TM}} \text { All } \\
\text { Blue Prestained Protein } \\
\text { Standards }\end{array}$ & Bio-Rad & 1610373 \\
\hline $\begin{array}{l}\text { ProLong Gold Antiface } \\
\text { Mountant with DAPI }\end{array}$ & Thermo Fisher Scientific & P36935 \\
\hline Proteinase K & VWR & $732-3276$ \\
\hline $\begin{array}{l}\text { Rhod-2 AM fluorescent } \\
\text { calcium indicator }\end{array}$ & Thermo Fisher Scientific & R1245MP \\
\hline $\begin{array}{l}\text { RIPA Lysis and Extraction } \\
\text { Buffer }\end{array}$ & Thermo Fisher Scientific & 89900 \\
\hline Roti-Histofix & Roth & P087.4 \\
\hline ROX reference dye & Thermo Fisher Scientific & 12223012 \\
\hline $\begin{array}{l}\mathrm{RT}^{2} \text { SYBR Green } \mathrm{qPCR} \\
\text { Mastermix }\end{array}$ & Qiagen & 330503 \\
\hline Sodium Chloride ( $\mathrm{NaCl}$ ) & Roth & 3957.1 \\
\hline Sodium dodecyl sulfate (SDS) & Roth & 2326.1 \\
\hline $\begin{array}{l}\text { Sodium hydrogen carbonate } \\
\left(\mathrm{NaHCO}_{3}\right)\end{array}$ & Roth & HN01.1 \\
\hline $\begin{array}{l}\text { Sodium hydroxide solution } \\
\text { (NaOH) }\end{array}$ & Roth & K021.1 \\
\hline $\begin{array}{l}\text { Superscript IV reverse } \\
\text { transcriptase }\end{array}$ & Thermo Fisher Scientific & 18090010 \\
\hline Taurine & Roth & 4721.1 \\
\hline $\begin{array}{l}\text { Tetramethylethylenediamine } \\
\text { (Temed) }\end{array}$ & Bio-Rad & 1610800 \\
\hline $\begin{array}{l}\text { Tris(hydroxymethyl)- } \\
\text { aminomethane (TRIS) }\end{array}$ & Roth & 5429.3 \\
\hline Triton X-100 & Roth & 3051.4 \\
\hline Trypsin $(2,5 \%)$ & Thermo Fisher Scientific & 15090046 \\
\hline Tween-20 & Roth & 9127.1 \\
\hline Xylene & J.T.Baker & 3410 \\
\hline
\end{tabular}




\subsection{Buffers and solutions}

Table 5: Buffers and solutions

\begin{tabular}{|c|c|}
\hline Buffer / solution & Ingredients \\
\hline \multicolumn{2}{|l|}{ Gel electrophoresis } \\
\hline TBE (5x Stock) & $\begin{array}{l}54 \mathrm{~g} \text { Tris } \\
27.5 \mathrm{~g} \text { boric acid } \\
3.72 \mathrm{~g} \text { EDTA } \\
\text { Dissolved in } 1 \mathrm{I} \mathrm{dd}_{2} \mathrm{O}\end{array}$ \\
\hline \multicolumn{2}{|l|}{ Immunocytochemistry } \\
\hline Blocking buffer & $2 \%$ BSA in PBS \\
\hline Staining buffer & $1 \% \mathrm{BSA}$ in PBS \\
\hline Cell permeabilisation buffer & $0.1 \%$ Triton-X 100 in $1 \%$ BSA in PBS \\
\hline \multicolumn{2}{|l|}{ Immunoblot } \\
\hline $2 \mathrm{M} \mathrm{DTT}$ & $308.5 \mathrm{mg}$ diluted in $1 \mathrm{ml} \mathrm{ddH_{2 } \mathrm { O }}$ \\
\hline 10x TBS & 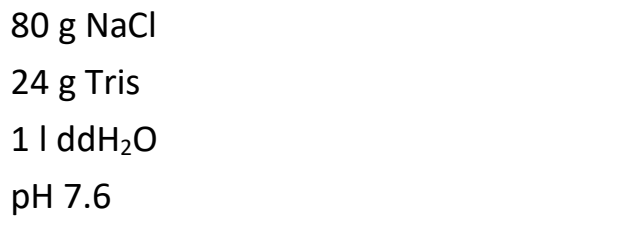 \\
\hline $1 \times$ TBST & 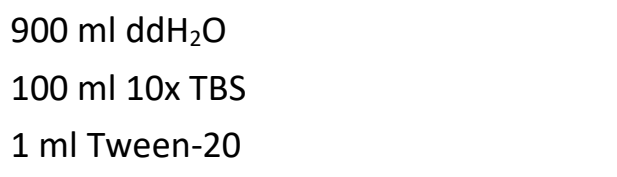 \\
\hline 10x Running buffer & $\begin{array}{l}60.4 \mathrm{~g} \text { Tris } \\
288 \mathrm{~g} \text { Glycine } \\
20 \mathrm{~g} \mathrm{SDS} \\
2 \mathrm{I} \mathrm{H}_{2} \mathrm{O}\end{array}$ \\
\hline Blocking buffer & $5 \%$ milk or $5 \%$ BSA in TBST \\
\hline RIPA buffer & $\begin{array}{l}10 \mathrm{ml} \text { RIPA Lysis and Extraction buffer } \\
1 \text { Pierce Protease Inhibitor Mini Tablet }\end{array}$ \\
\hline \multicolumn{2}{|l|}{ Confocal calcium Imaging } \\
\hline Tyrode's solution (10x stock) & 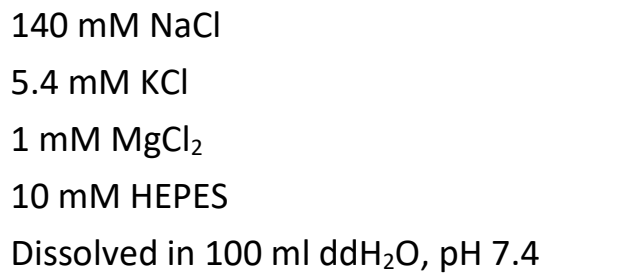 \\
\hline
\end{tabular}




\begin{tabular}{|c|c|}
\hline Tyrode's solution ( $1 \mathrm{x})$ & 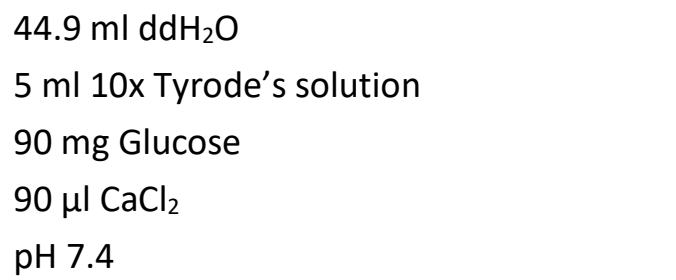 \\
\hline Rhod-2 AM stock solution & $\begin{array}{l}50 \mu \mathrm{g} \text { Rhod-2 } \\
44 \mu \mathrm{l} \text { DMSO }\end{array}$ \\
\hline Rhod-2 AM staining solution & $\begin{array}{l}2 \mu \mathrm{l} \text { Rhod-2 AM stock solution } \\
0.5 \mu \mathrm{l} \text { Pluronic F-127 } \\
400 \mu \mathrm{l} \text { Tyrode's solution (1x) }\end{array}$ \\
\hline Helothermine (40 mM stock) & $\begin{array}{l}100 \mu g \text { Helothermine } \\
98.5 \mu \mathrm{lddH} \mathrm{d}_{2} \mathrm{O}\end{array}$ \\
\hline \multicolumn{2}{|c|}{ Langendorff cardiomyocyte isolation } \\
\hline Tyrode's solution $(1 \mathrm{x})$ & $\begin{array}{l}113 \mathrm{mM} \mathrm{NaCl} \\
4.7 \mathrm{mM} \mathrm{KCl} \\
0.6 \mathrm{mM} \mathrm{KH}_{2} \mathrm{PO}_{4} \\
0.6 \mathrm{mM} \mathrm{Na}_{2} \mathrm{HPO}_{4} \times 2 \mathrm{H}_{2} \mathrm{O} \\
1.2 \mathrm{mM} \mathrm{MgSO}_{4} \times 7 \mathrm{H}_{2} \mathrm{O} \\
12 \mathrm{mM} \mathrm{NaHCO}_{3} \\
10 \mathrm{mM} \mathrm{KHCO}_{3} \\
10 \mathrm{mM} \mathrm{HEPES} \\
30 \mathrm{mM} \text { Taurine } \\
5.5 \mathrm{mM} \text { Glucose } \\
10 \mathrm{mM} \text { 2,3-butanedione monoxime (BDM) } \\
\text { Dissolved in dd }{ }_{2} \mathrm{O}, \mathrm{pH} 7.42 \text { at } 36.5^{\circ} \mathrm{C}\end{array}$ \\
\hline Digestion buffer & $\begin{array}{l}20 \text { ml Tyrode's solution } \\
300 \mu \text { l Liberase } \\
111.2 \mu \mathrm{l} \text { Trypsin (2.5\%) } \\
25 \mu \mathrm{l} 10 \mathrm{mM} \mathrm{CaCl}_{2}\end{array}$ \\
\hline Stop solution 1 & $\begin{array}{l}2.25 \mathrm{ml} \text { Tyrode's solution } \\
250 \mu \mathrm{l} \mathrm{BCS} \\
3.125 \mu \mathrm{l} 10 \mathrm{mM} \mathrm{CaCl}_{2}\end{array}$ \\
\hline Stop solution 2 & $\begin{array}{l}23.75 \mathrm{ml} \text { Tyrode's solution } \\
1.25 \mathrm{ml} \mathrm{BCS}\end{array}$ \\
\hline
\end{tabular}




\begin{tabular}{|c|c|}
\hline \multicolumn{2}{|c|}{ Epifluorescence calcium-transient measurements } \\
\hline Calcium reloading & $\begin{aligned} & 0.1 \mathrm{mmol} / \mathrm{l}: 5 \mathrm{ml} \text { stop solution } 2 \\
& 5 \mu \mathrm{l} 100 \mathrm{mM} \mathrm{CaCl} 2 \\
& 0.2 \mathrm{mmol} / \mathrm{l}: 5 \mathrm{ml} \text { stop solution } 2 \\
& 10 \mu \mathrm{l} 100 \mathrm{mM} \mathrm{CaCl} 2 \\
& 0.4 \mathrm{mmol} / \mathrm{l}: 5 \mathrm{ml} \text { stop solution } 2 \\
& 20 \mu \mathrm{l} 100 \mathrm{mM} \mathrm{CaCl} 2 \\
& 0.8 \mathrm{mmol} / \mathrm{l}: \begin{array}{l}10 \mathrm{ml} \text { stop solution } 2 \\
80 \mu \mathrm{l} 100 \mathrm{mM} \mathrm{CaCl} 2\end{array}\end{aligned}$ \\
\hline Epifluorescence Tyrode (10x stock) & $\begin{array}{l}40 \mathrm{mM} \mathrm{KCl} \\
1400 \mathrm{mM} \mathrm{NaCl} \\
10 \mathrm{mM} \mathrm{MgCl}_{2} \\
10 \mathrm{mM} \mathrm{HEPES} \\
\text { Dissolved in } 1 \text { I dd } \mathrm{d}_{2} \mathrm{O}, \mathrm{pH} 7\end{array}$ \\
\hline Epifluorescence tyrode $(1 \mathrm{x})$ & 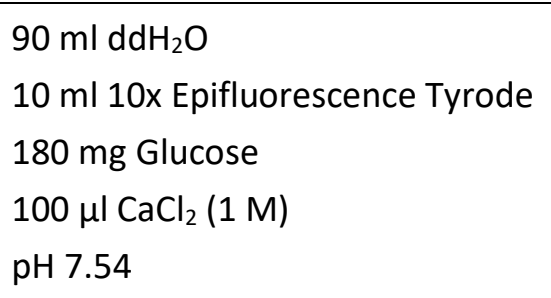 \\
\hline Fura-2 AM stock solution & $\begin{array}{l}50 \mu \mathrm{g} \text { Fura-2 } \\
44.4 \mu \mathrm{l} \text { DMSO }\end{array}$ \\
\hline Fura-2 AM staining solution & $\begin{array}{l}5 \mu \mathrm{l} \text { Fura-2 AM stock solution } \\
1 \mu \mathrm{l} \text { Pluronic F-127 } \\
500 \mu \mathrm{l} \text { Epifluorescence Tyrode (1x) }\end{array}$ \\
\hline
\end{tabular}

\subsection{Kits}

Table 6: Kits

\begin{tabular}{|l|l|l|}
\hline \multicolumn{1}{|c|}{ Product } & \multicolumn{1}{|c|}{ Manufacturer } & \multicolumn{1}{c|}{ Catalogue number } \\
\hline $\begin{array}{l}\text { P3 Primary Cell 4D- } \\
\text { Nucleofector X }\end{array}$ & Lonza & V4XP-3024 \\
\hline Picro Sirius Red Stain Kit & Abcam & Ab150681 \\
\hline Pierce BCA Protein Assay Kit & Thermo Fisher Scientific & 23227 \\
\hline QIAamp DNA Mini Kit & Qiagen & 51306 \\
\hline QIAquick Gel Extraktion Kit & Qiagen & 28706 \\
\hline QuantiFluor dsDNA System & Promega & E2670 \\
\hline RNeasy Fibrous Tissue Mini Kit & Qiagen & 74704 \\
\hline RT2 First Strand Kit & Qiagen & 330404 \\
\hline
\end{tabular}




\begin{tabular}{|l|l|l|}
\hline $\begin{array}{l}\text { SuperSignal West Femto } \\
\text { Maximum Sensitivity Substrate }\end{array}$ & Thermo Fisher Scientific & 34094 \\
\hline $\begin{array}{l}\text { TGX Stain-Free FastCast } \\
\text { Acrylamide Starter Kit 10\% }\end{array}$ & Bio-Rad & 1610182 \\
\hline $\begin{array}{l}\text { Trans-Blot Turbo RTA Mini } \\
\text { Nitrocellulose Transfer Kit }\end{array}$ & Bio-Rad & 1704270 \\
\hline $\begin{array}{l}\text { TruSeq RNA Sample } \\
\text { Preparation V2 Kit }\end{array}$ & Illumina & RS-122-2002 \\
\hline
\end{tabular}

\subsection{Antibodies}

Table 7: Primary antibodies

\begin{tabular}{|l|l|l|l|l|}
\hline \multicolumn{1}{|c|}{ Antibody } & \multicolumn{1}{|c|}{ Origin } & \multicolumn{1}{|c|}{ Dilution } & \multicolumn{1}{c|}{ Manufacturer } & \multicolumn{1}{c|}{$\begin{array}{c}\text { Catalogue } \\
\text { number }\end{array}$} \\
\hline$\alpha$-Actinin & Mouse & $1: 1000$ & Sigma Aldrich & A7811 \\
\hline Connexin43 & Rabbit & $1: 1000$ & Abcam & ab11370 \\
\hline CRISPLD1 & Mouse & $1: 500$ & Abnova & H00083690-B01P \\
\hline CRISPLD1 & Rabbit & $1: 250$ & Sigma Aldrich & HPA024725 \\
\hline CRISPLD1 & Rabbit & $1: 1000$ & Abcam & ab123039 \\
\hline CRISPLD1 & Rabbit & $1: 500$ & $\begin{array}{l}\text { Antibodies-online } \\
\text { (Abbexa) }\end{array}$ & $\begin{array}{l}\text { ABIN6058851/ } \\
\text { abx301729 }\end{array}$ \\
\hline $\begin{array}{l}\text { Cardiac Troponin } \\
\text { T (cTNT) }\end{array}$ & Mouse & $1: 200$ & $\begin{array}{l}\text { Thermo Fisher } \\
\text { Scientific }\end{array}$ & MA5-12960 \\
\hline $\begin{array}{l}\text { Myosin Light } \\
\text { Chain 2 (MLC2V) }\end{array}$ & Rabbit & $1: 200$ & Proteintech & 10906-1-AP \\
\hline RYR2 & Rabbit & $1: 500$ & Sigma Aldrich & HPA020028 \\
\hline
\end{tabular}

Table 8: Secondary antibodies, dyes and conjugates

\begin{tabular}{|l|l|l|l|l|}
\hline \multicolumn{1}{|c|}{ Antibody } & \multicolumn{1}{|c|}{ Origin } & \multicolumn{1}{c|}{ Dilution } & \multicolumn{1}{c|}{ Manufacturer } & \multicolumn{1}{c|}{$\begin{array}{c}\text { Catalogue } \\
\text { number }\end{array}$} \\
\hline Anti-rabbit HRP & Donkey & $1: 10000$ & Th. Geyer & NA934 \\
\hline Anti-mouse HRP & Sheep & $1: 10000$ & Th. Geyer & NA931 \\
\hline $\begin{array}{l}\text { Goat anti-mouse } \\
555\end{array}$ & Goat & $1: 1000$ & Abcam & ab150114 \\
\hline $\begin{array}{l}\text { Goat anti-rabbit } \\
488\end{array}$ & Goat & $1: 500$ & $\begin{array}{l}\text { Thermo Fisher } \\
\text { Scientific }\end{array}$ & A11008 \\
\hline Hoechst33342 & - & $1: 1000$ & $\begin{array}{l}\text { Thermo Fisher } \\
\text { Scientific }\end{array}$ & H3570 \\
\hline
\end{tabular}




\begin{tabular}{|l|l|l|l|l|}
\hline $\begin{array}{l}\text { WGA488 } \\
\text { conjugate }\end{array}$ & - & $10 \mu \mathrm{g} / \mathrm{ml}$ & $\begin{array}{l}\text { Thermo Fisher } \\
\text { Scientific }\end{array}$ & W11261 \\
\hline
\end{tabular}

\subsection{Primers}

Table 9: Genotyping primers

\begin{tabular}{|l|l|}
\hline Primername & Sequence $\mathbf{( 5}^{\prime}$ - $\mathbf{3}^{\prime}$ ) \\
\hline CMVCre_F & ATGCTTCTGTCCGTTTGCCG \\
\hline CMVCre_R & CCTGTTTTGCACGTTCACCG \\
\hline CSD-F & GGAATAGGCGTAATGATGTCTATAAGC \\
\hline CSD-F2 & GGAGCAGTTTGCCATCTTTGAA \\
\hline CSD-LoxF & GAGATGGCGCAACGCAATTAATG \\
\hline CSD-neoF & GGGATCTCATGCTGGAGTTCTTCG \\
\hline CSD-R & GAAGAACATTTACCTACCCACACATCC \\
\hline CSD-R2 & GCCTCCTCCAAAACTGGGTG \\
\hline CSD-ttR & AAGTAAATGGGCAATGCTCTGAGCTG \\
\hline FlpO_F & GCTATCGAATTCCACCATGGCTCCTAAGAAGAA \\
\hline FlpO_R & CAATGCGATGAATTCTCAGATCCGCCTGTTGATGTA \\
\hline Myh6Cre-ctrI_F & CAAATGTTGCTTGTCTGGTG \\
\hline Myh6Cre-ctrl_R & GTCAGTCGAGTGCACAGTTT \\
\hline Myh6Cre-F & ATGACAGACAGATCCCTCCTATCTCC \\
\hline Myh6Cre-R & CTCATCACTCGTTGCATCATCGAC \\
\hline & \\
\hline
\end{tabular}

Table 10: RT-qPCR primers

\begin{tabular}{|c|c|c|}
\hline Primername & $\begin{array}{l}\text { Sequence Forward }\left(5^{\prime}-3^{\prime}\right) \text { or } \\
\text { supplier }\end{array}$ & $\begin{array}{c}\text { Sequence Reverse }\left(5^{\prime}-3^{\prime}\right) \text { or } \\
\text { ordernumber }\end{array}$ \\
\hline Hs_18S & GACACGGACAGGATTGACAG & CTAGTTAGCATGCCAGAGTCTC \\
\hline Hs_CRISPLD1 & TGCCCAAGAGTATACTGTCCT & GATTTCGAACCACTCCAGCA \\
\hline Hs_CRISPLD1_Ex2-3_2 & AGGTGTATCCAACAGCCTCTA & CCCAGTGTGCTCCCAAATTC \\
\hline Hs_CRISPLD1_F2 & GTCACAAGTCCATGACACCCA & CTATCCAAACAGCCAGCAGGA \\
\hline Hs_GAPDH & GTTCGTCATGGGTGTGAACC & GGTCATGAGTCCTTCCACGA \\
\hline AGTR1 & Qiagen & PPH02362F \\
\hline ATP1A2 & Qiagen & PPH09011A \\
\hline CACNA2D3 & Qiagen & PPH20424B \\
\hline CACNAG4 & Qiagen & PPH17057A \\
\hline
\end{tabular}




\begin{tabular}{|l|l|l|}
\hline CRISPLD1 & Qiagen & PPH20603A \\
\hline RYR2 & Qiagen & PPH10458A \\
\hline sCN5A & Qiagen & PPH01671F \\
\hline Cre_qPCR_1 & CGGTCTGGCAGTAAAAACTAT & CAGGGTGTTATAAGCAATCCC \\
\hline Ms_18S_1 & CATGCATGTCTAAGTACGCAC & GTCGGCATGTATTAGCTCTAG \\
\hline Ms_ATP2A2_origene_1 & GTGAAGTGCCATCAGTATGACGG & GTGAGAGCAGTCTCGGTAGCTT \\
\hline Ms_CRISPDL1_ex13-14_1 & GCTCGTGTAATCGGGACCAG & CCACAGGCATGACATCCACA \\
\hline Ms_CRISPLD1_ex2-3_1 & AAGTCAGGTGTACCCAACAGC & ATTCTGTCCAATGGAGGGCAG \\
\hline Ms_CRISPLD1_ex5-6_1 & TAGGCTGTGCCGTCAATCTG & AAGCAGAACAAGGCCTTCCA \\
\hline Ms_GAPDH_1 & GAGACGGCCGCATCTTCTT & CAATCTCCACTTTGCCACTGC \\
\hline Ms_Myh6_origene_1 & GCTGGAAGATGAGTGCTCAGAG & CCAGCCATCTCCTCTGTTAGGT \\
\hline Ms_Myh7_origene_1 & GCTGGAAGATGAGTGCTCAGAG & TCCAAACCAGCCATCTCCTCTG \\
\hline Ms_nppa_2 & CTGCTTCGGGGGTAGGATTG & GCTCAAGCAGAATCGACTGC \\
\hline Ms_nppb_1 & CAGCTCTTGAAGGACCAAGG & ACTTCAGTGCGTTACAGCCC \\
\hline ms_CRISPLD1_ex4-6_3 & CACACTGGGGAAGATACCGA & ACCAGTTTCCCCTGAGTGTA \\
\hline
\end{tabular}

Table 11: Primers for CRISPLD1 KO Validation

\begin{tabular}{|l|l|}
\hline Primer name & Sequence $\mathbf{( 5}^{\prime} \mathbf{}^{\mathbf{3}} \mathbf{)}$ \\
\hline CRISPLD1_gDNA_F & GAATATTCCATGGCAGAGTTTGG \\
\hline CRISPLD1_gDNA_R & GGTTGTTCCTTTGCACTGATCT \\
\hline CRISPLD1_gDNA_R3 & TGAATAGTGTGCAGTGAGAAGTG \\
\hline CRISPLD1_I1_ex3_F & GAATTTGGGAGCACACTGGG \\
\hline CRISPLD1_I1\&3_ex10_R & ATCTACCCAGCCACCATCAT \\
\hline
\end{tabular}

\subsection{Devices}

Table 12: Devices

\begin{tabular}{|l|l|l|}
\hline \multicolumn{1}{|c|}{ Application } & \multicolumn{1}{c|}{ Device } & \multicolumn{1}{c|}{ Manufacturer } \\
\hline \multirow{2}{*}{ Cell counting } & $\begin{array}{l}\text { Thoma new counting chamber } \\
\text { Countess II FL Automated Cell } \\
\text { Counter } \\
\text { CASY Model TT }\end{array}$ & $\begin{array}{l}\text { Roth } \\
\text { Thermo Fisher Scientific } \\
\text { Innovatis }\end{array}$ \\
\hline Cell culture incubator & BBD 6220 & Thermo Fisher Scientific \\
\hline Cell Freezing & Mr. Frosty & Nalgene \\
\hline Cell stimulator & MyoPacer cell stimulator & IonOptix \\
\hline
\end{tabular}


Materials and Methods

\begin{tabular}{|c|c|c|}
\hline Centrifuges & $\begin{array}{l}\text { Centrifuge 5418R } \\
\text { Heraeus Fresco } 21 \\
\text { Mini-Centrifuge Sprout } \\
\text { Multifuge X1R }\end{array}$ & $\begin{array}{l}\text { Eppendorf } \\
\text { Thermo Fisher Scientific } \\
\text { Sprout } \\
\text { Thermo Fisher Scientific }\end{array}$ \\
\hline Controlled rate freezer & KRYO 560-16 & Planer \\
\hline Electrophoresis & $\begin{array}{l}\text { BioDoc Analyze Darkhood } \\
\text { EOS 1100D }\end{array}$ & $\begin{array}{l}\text { Biometra } \\
\text { Canon }\end{array}$ \\
\hline Embedding station & EG1150H & Leica \\
\hline Heating block & ThermoMixer F1.5 & Eppendorf \\
\hline Histology sections & Microtome RM 2165 & Leica \\
\hline Imaging & $\begin{array}{l}\text { LSM } 710 \\
\text { Primovert }\end{array}$ & Zeiss \\
\hline Immunoblotting & $\begin{array}{l}\text { Trans-Blot Turbo Transfer } \\
\text { System } \\
\text { ChemiDoc XRS+ }\end{array}$ & Bio-Rad \\
\hline Magnetic stir & $\mathrm{RH}$ basic & IKA \\
\hline mRNA sequencing & $\begin{array}{l}\text { Bioanalyzer } 2100 \\
\text { cBot } \\
\text { HiSeq2000 }\end{array}$ & $\begin{array}{l}\text { Agilent } \\
\text { Illumina } \\
\text { Illumina }\end{array}$ \\
\hline PCR Cycler & $\begin{array}{l}\text { Mastercycler nexus Gradient } \\
\text { Mastercycler nexus X2 Eco }\end{array}$ & eppendorf \\
\hline pH Measurement & FiveEasy F20 & Mettler-Toledo \\
\hline Power supply & PowerPac Basic Power Supply & Bio-Rad \\
\hline Real Time PCR cycler & $\begin{array}{l}\text { CFX96 Touch Real-Time PCR } \\
\text { Detection System } \\
\text { 7900HT Fast Real-Time PCR } \\
\text { System }\end{array}$ & $\begin{array}{l}\text { Bio-Rad } \\
\text { Applied Biosystems }\end{array}$ \\
\hline Spectrophotometer & NanoDrop One & Thermo Fisher Scientific \\
\hline Sterile working bench & Safe 2020 & Thermo Fisher Scientific \\
\hline Tissue Lyser & Tissue Lyser LT & Qiagen \\
\hline Tissue processor & TP1020 & Leica \\
\hline Transfection unit & $\begin{array}{l}\text { Amaxa Nucleofector II device } \\
\text { 4D-Nucleofector Core Unit }\end{array}$ & Lonza \\
\hline Vortexer & Unitexer1 & LLG \\
\hline Water bath & Waterbath WNB7 & Memmert \\
\hline Balances & $\begin{array}{l}\text { ENTRIS822-1S } \\
\text { QUINTIX124-S } \\
\text { SECURA225D-1S }\end{array}$ & Sartorius \\
\hline
\end{tabular}




\subsection{Software}

Table 13: Software

\begin{tabular}{|l|l|l|}
\hline \multicolumn{1}{|c|}{ Product } & \multicolumn{1}{c|}{ Version } & Manufacturer \\
\hline ABI 7900 HT SDS & 2.4 & Applied Biosystems \\
\hline Adobe Illustrator 2020 & 24.3 & Adobe Systems \\
\hline BioDoc Analyze & 2.66 .3 .44 & Biometra \\
\hline BioRad CFX Maestro & 4.0 .2325 .0418 & Bio-Rad \\
\hline BioRender & 2021 & BioRender.com \\
\hline Chromas & 2.6 .4 & Technelysium Pty Ltd \\
\hline & & $\begin{array}{l}\text { Institute for Genomics and } \\
\text { Bioinformatics } \\
\text { Christian Doppler Laboratory } \\
\text { for Genomics and } \\
\text { Bioinformatics } \\
\text { Graz University of Technology }\end{array}$ \\
\hline ClueGO & V2.5.8 & Cytoscape Team \\
\hline Cytoscape & & Microsoft \\
\hline Excel & 3.7 .2 & GraphPad Software \\
\hline GraphPad Prism 8 & 2013 & Wayne Rasband \\
\hline ImageJ & 8.4 .2 & Bio-Rad \\
\hline ImageLab & $1.51 \mathrm{n}$ & lonOptix LLC \\
\hline IonWizard & 5.2 .1 & ADInstruments \\
\hline LabChart Pro & 6.4 revision 1 & Visual Sonics \\
\hline VevoLab & V8.0.5 & Microsoft \\
\hline Word & 3.1 .1 & Carl Zeiss \\
\hline Zen & 2013 & \\
\hline & 2.3 & \\
\hline
\end{tabular}

\subsection{Animal experiments}

\subsubsection{Animal welfare}

All animal experiments were approved by LAVES and performed in agreement with institutional and governmental guidelines. The mice were maintained in Individually Ventilated Cages (IVC) under laboratory standard conditions with a light/dark cycling of $12 \mathrm{~h}$. They could access water and food ad libitum. Animals were kept in groups up to 5 and allowed to habituate at a minimum of $24 \mathrm{~h}$ prior to experimental use. 


\subsubsection{Generation of mouse strains}

Two knockout (KO) mouse lines were used in this study, a conditional and a global KO. To generate the Crispld1 KO lines, CRISPLD1 ${ }^{\text {tm1a(KOMP)Wtsi }}$ was crossed with a FLP delete mouse line (CAG-FlpO) to remove the selection cassette. After backcrossing with C57BL/6N mice, delivered from Charles River and crossing of heterozygous Crispld1 KO lines, the conditional KO mouse line CRISPLD1 ${ }^{\mathrm{fl} / \mathrm{fl}}$ with the coding exon 5 flanked by loxP sites was obtained. The Crispld1 alleles were genotyped using primers CSD-F and CSD-ttR. PCR amplified 514 bp from the wildtype and $369 \mathrm{bp}$ from the floxed allele.

CRISPLD1 ${ }^{\mathrm{fl} / \mathrm{fl}}$ were either crossed with $\mathrm{Myh}^{\mathrm{Cre+}}$ to generate Myh6 ${ }^{\mathrm{Cre}+}$; CRISPLD1 $1^{\mathrm{fl} / \mathrm{fl}}$ mice or with a $\mathrm{CMV}^{\mathrm{Cre}+}$ to obtain $\mathrm{CMV}^{\mathrm{Cre}+}$; CRISPLD1 $1^{\mathrm{fl} / \mathrm{fl}}$ mice

\subsubsection{Echocardiography}

Heart function of WT and KO mouse lines were examined by transthoracic echocardiography using the Vevo 2100 system as previously described (Pistner et al., 2010). Mice were anesthetized with isoflurane $(1 \%-2 \%)$ using a respiratory mask and their thorax depilated. The heart was recorded in the B-Mode or M-Mode in the long axis or short axis view. During the recordings the vital parameters of the mouse was monitored. For analysis, the LV-trace function of the VevoLab Software was used on the M-Mode images to measure anterior wall thickness (AWThd/s), posterior wall thickness (PWThd/s), left ventricular end-diastolic diameter (LVEDD), left ventricular end-systolic diameter (LVESD) and heart rate. These measurements were used to calculate ejection fraction (EF) and fractional shortening (FS). Data was visualized with GraphPad Prism. For statistical analysis, one-way ANOVA with Tukey post-hoc test $(p<0.05)$ was used.

Echocardiography was performed by the SFB1002 service team of Marcel Zoremba, Roland Blume and Sabrina Koszewa (Cardiology and Pneumology, University Medical Center Göttingen).

\subsubsection{Heart dissections}

Mice were anesthetized with isoflurane and sacrificed by cervical dislocation. Before dissection, the body weight was measured. The thorax was cut open and the heart excised at the aorta and transferred into a falcon filled with 1x PBS and weighted. A 21 gauge blunt needle was placed into the aorta and fixed with a clamp. The heart was perfused with PBS to remove residual blood. The atria as well as either the tip of the heart or the right ventricle 
were removed. Left ventricle was weighted again before all samples were snap frozen in liquid nitrogen. The lung was dissected and weighted, and the tibia removed and measured using an electronic gauge.

\subsection{Cell biological methods}

\subsubsection{Cultivation and maintenance of iPSCs}

iPSC lines were maintained as described before by Kleinsorge and Cyganek with minor modifications (Kleinsorge \& Cyganek, 2020). Briefly, $1 \mathrm{ml}$ Matrigel (Table 3) was added to culture dishes and incubated for $30 \mathrm{~min}$ at $37^{\circ} \mathrm{C}$ or overnight at $4{ }^{\circ} \mathrm{C}$ before dishes were used for cell culture. For preparation of immunostainings, glass cover slips were incubated in $0.1 \%$ $\mathrm{HCl}$ overnight and washed with distilled water. Following incubation in $70 \%$ ethanol for a minimum of $12 \mathrm{~h}$, the cover slips were cleaned with lint-free papers and sterilized at $200{ }^{\circ} \mathrm{C}$ for $2 \mathrm{~h}$. The glass cover slips were coated with $550 \mu$ l Matrigel and incubated for $45 \mathrm{~min}$ at 37 ${ }^{\circ} \mathrm{C}$ or overnight at $4{ }^{\circ} \mathrm{C}$ before usage.

iPSCs cells were passaged to a new Matrigel coated plate at a confluency of $80-90 \%$ iPSC. For this, the cells were washed twice and incubated with prewarmed Versene solution for 3-5 min. Versene solution was removed and the cells were resuspended in StemFlex supplemented with $2 \mu \mathrm{M}$ TZV. Matrigel was removed from the prepared culture dishes and the cells were transferred into lower densities onto the dishes. After $24 \mathrm{~h}$ of incubation, medium was replaced by StemFlex only. For cardiac differentiation, iPSC passages 18-40 were used.

\subsubsection{Directed differentiation of iPSCs into iPSC-CMs}

The human iPSC line ipWT1.3 used in this study was generated from a healthy donor and provided by the Stem Cell Unit (UMG Göttingen). IpWT1.3 (GOEi014-B.3; origin: dermal fibroblast) was reprogrammed under feeder-free culture conditions with integration-free episomal 4-in-1 CoMiP reprogramming plasmid (Addgene, catalog 63726) as described previously (Cyganek et al., 2018, El-Battrawy et al., 2018). Cells were cultured in StemFlex Medium on Matrigel (diluted 1:60-1:120) coated plates. Differentiation into CMs was started at a confluence of 70-90\%. Cells were treated with Cardio Differentiation Medium (see Table 3) supplemented with $6 \mu \mathrm{M}$ CHIR99021 for $48 \mathrm{~h}$. Afterwards, the medium was changed to Cardio Differentiation Medium supplemented with $5 \mu \mathrm{M}$ IWP2 for further $48 \mathrm{~h}$. The medium was changed to Cardio Culture on day 7. Metabolic selection was performed on day 14 with 
Cardio Selection Medium for 4 days. On day 18, the cells were digested with $0.25 \%$ Trypsin/EDTA to obtain lower cell densities. Afterwards, CMs were cultured for another 4 days with Cardio Selection Medium before changing to Cardio Culture Medium for further cultivation until day 60. Differentiation efficiency was analyzed by observation of the contracting cells.

\subsubsection{Digestion and selection of iPSC-CMs}

To achieve lower cell densities and to obtain a purified cardiomyocyte population, iPSC-CMs were digested followed by metabolic selection as described previously (Kleinsorge \& Cyganek, 2020). For this, iPSC-CMs on day 14 were washed once with Versene solution and incubated with prewarmed $0.25 \%$ Trypsin-EDTA for $10 \mathrm{~min}$ at $37^{\circ} \mathrm{C}$. Afterwards, the cells were flushed off by adding $2 \mathrm{ml}$ of cardio digestion medium (Table 3) and transferred to a $15 \mathrm{ml}$ falcon. After centrifugation at $100 \times \mathrm{g}$ for $10 \mathrm{~min}$, the supernatant was removed, the cells were resuspended in cardio digestion medium and transferred onto Matrigel coated culture dishes. Medium was changed to Cardio Selection Medium (Table 3) after 2 days and changed every 2-3 days for a total of 1 week. Afterwards cells were maintained in Cardio Culture Medium until day 60 .

\subsubsection{Freezing and thawing of iPSC and iPSC-CMs}

Cryopreservation and recovery of iPSC and iPSC-CMs was performed according to Kleinsorge and Cyganek with minor changes (Kleinsorge \& Cyganek, 2020). In detail, the cells were washed once and incubated for 3 min with prewarmed Versene solution. The solution was removed and cells were resuspended in StemFlex medium supplemented with $4 \mu M$ TZV and $20 \%$ DMSO and carefully transferred into a cryo tube. The cells were stored at $-80{ }^{\circ} \mathrm{C}$ for at least $2 \mathrm{~h}$ in a freezing container with isopropanol to allow a steady freezing rate of approximately $-1{ }^{\circ} \mathrm{C}$ per min.

For cryopreservation of IPSC-CMs, the cells were washed once with Versene solution and incubated with $0.25 \%$ Trypsin-EDTA for $10 \mathrm{~min}$ at $37^{\circ} \mathrm{C}$. iPSC-CMs were rinsed off using $1 \mathrm{ml}$ FBS and transferred into a $15 \mathrm{ml}$ falcon for centrifugation at $100 \mathrm{xg}$ for $10 \mathrm{~min}$. The medium was removed and the cells were resuspended in a small volume of FBS. Cells were counted using Countess II FL Automated Cell Counter, 2-2.5 Mio cells diluted in Cardio Preservation Medium (Table 3) and transferred into a cryo tube. The cryopreservation process was performed with a controlled rate freezer allowing controlled freezing of $-1{ }^{\circ} \mathrm{C}$ per min up 
to $-90^{\circ} \mathrm{C}$. Cardiomyocytes and iPSC cells were long term stored at $-150^{\circ} \mathrm{C}$.

For cell recovery, cryo tubes were thawed at $37{ }^{\circ} \mathrm{C}$ and cells were transferred into $10 \mathrm{ml}$ medium (Cardio Culture Medium for iPSC-CMs and StemFlex medium for iPSCs) and centrifuged at $200 \times \mathrm{g}$ for $5 \mathrm{~min}$. The supernatant was removed and iPSC cells were resuspended in $2 \mathrm{ml}$ StemFlex supplemented with $2 \mu \mathrm{M}$ TZV, whereas iPSC-CMs were resuspended in $2 \mathrm{ml}$ cardio digestion medium. The cells were plated onto Matrigel coated culture dishes and incubated at $37{ }^{\circ} \mathrm{C}$ and $5 \% \mathrm{CO}_{2}$.

\subsubsection{Generation and validation of KO-iPSC clones by CRISPR/Cas9}

Transfection of the wildtype iPSC line ipWT1.3 with CRISPLD1_Ex_CR2 (Table 14) targeting CRISPLD1 exon 7 was performed with the P3 Primary Cell 4D-Nucleofector X kit and the 4DNucleofector Core Unit. RNA oligos (Alt-R CRISPR-Cas9 crRNA and Alt-R CRISPR-Cas9 tracrRNA) were diluted to $100 \mu \mathrm{M}$ stock solution. $5 \mu \mathrm{l}$ of each was mixed, incubated at $95{ }^{\circ} \mathrm{C}$ for 5 min and cooled down before usage. The nucleofector mix was prepared by mixing $50 \mu \mathrm{M}$ CRISPRRNA (CRISPLD1_Ex_CR2) and $61 \mu \mathrm{M}$ Cas9 nuclease. The mixture was then incubated for 20 min at RT and pre-heated to $37^{\circ} \mathrm{C}$ before transfection.

iPSCs with 70-80\% confluency were pretreated with $2 \mu \mathrm{M}$ thiazovivin (TZV) for $20 \mathrm{~min}$. The medium was aspirated and the cells washed and dissolved for 6 min using Versene solution. The cells were washed of the plate using StemFlex medium and counted with the CASY cell counter. $2 \times 10^{6}$ cells were transferred into a falcon and centrifuged for $3 \mathrm{~min}$ at $200 \mathrm{~g}$. Finally, the cells were resuspended in the nucleofector and electroporated using the program CA-137. Subsequently, the cells were diluted in $300 \mu \mathrm{l}$ StemFlex and replated onto a 6-well plate containing StemFlex medium, $2 \mu \mathrm{M}$ TZV and 1x Pen/Strep. $24 \mathrm{~h}$ after transfection, the medium was changed and 2 days after transfection the cells were singularized for analysis. The iPSC clones were expanded and analyzed at genomic level via PCR and Sanger sequencing. The QIAamp DNA Mini kit was used for DNA isolation. PCR reactions were performed using the primers CRISPLD1_gDNA_F and CRISPLD1_gDNA_R3 or CRISPLD1_gDNA_F and CRISPLD1_gDNA_R according to Table 15 and Table 16. These PCR products were subsequently purified after agarose gel electrophoresis using the QIAquick Gel extraction kit (see 2.13.6 and 2.13.7) and validated by sequencing (SeqLab, Göttingen). 
Table 14: Guide RNA used for CRISPR/Cas9 genome editing

\begin{tabular}{|l|l|}
\hline \multicolumn{1}{|c|}{ Name } & \multicolumn{1}{c|}{ Sequence (5'-3') } \\
\hline CRISPLD1_Ex_CR2 & CCATGTCCGGACAAGATCAGA \\
\hline
\end{tabular}

Table 15: Standard PCR reaction for KO clone validation on gDNA level

\begin{tabular}{|l|l|}
\hline \multicolumn{1}{|c|}{ Reagents } & \multicolumn{1}{c|}{ Volume in $\mu \mathrm{l}$} \\
\hline Phire Buffer & 4.0 \\
\hline dNTPs $(10 \mathrm{mM})$ & 0.5 \\
\hline Forward Primer $(10 \mathrm{nmol} / \mu \mathrm{l})$ & 1.0 \\
\hline Reverse Primer $(10 \mathrm{nmol} / \mu \mathrm{l})$ & 1.0 \\
\hline Phire Polymerase & 0.4 \\
\hline $\mathrm{H}_{2} \mathrm{O}$ & Up to $20 \mu \mathrm{l}$ \\
\hline DNA & $100 \mathrm{ng}$ \\
\hline
\end{tabular}

Table 16: Standard PCR protocol for KO clone validation on gDNA level

\begin{tabular}{|l|l|l|}
\hline \multicolumn{1}{|c|}{ Step } & \multicolumn{1}{|c|}{ Temperature $\left({ }^{\circ} \mathrm{C}\right)$} & \multicolumn{1}{c|}{ Time } \\
\hline Denaturation & 98 & $5 \mathrm{~min}$ \\
\hline \multirow{3}{*}{$\begin{array}{l}\text { Amplification } \\
(x 35)\end{array}$} & 98 & $5 \mathrm{sec}$ \\
\cline { 2 - 4 } & 65 & $30 \mathrm{sec}$ \\
\hline Elongation & 72 & $1: 30 \mathrm{~min}$ \\
\hline
\end{tabular}

\subsubsection{Validation of KO-iPSC clones on cDNA level}

The validation of CRISPLD1 KO on cDNA level was performed with PCR according to Table 17 and Table 18. The PCR product was analyzed by gel electrophoresis (see 2.13.6).

Table 17: Standard PCR reaction for KO clone validation on cDNA level

\begin{tabular}{|l|l|}
\hline \multicolumn{1}{|c|}{ Reagents } & \multicolumn{1}{c|}{ Volume in $\mu \mathrm{l}$} \\
\hline $5 x$ Green GoTaq buffer & 5.0 \\
\hline dNTPs $(10 \mathrm{mM})$ & 1.6 \\
\hline Forward Primer $(10 \mathrm{nmol} / \mu \mathrm{l})$ & 1.0 \\
\hline Reverse Primer $(10 \mathrm{nmol} / \mu \mathrm{l})$ & 1.0 \\
\hline GoTaq Polymerase & 0.1 \\
\hline $\mathrm{H}_{2} \mathrm{O}$ & Up to $20 \mu \mathrm{l}$ \\
\hline DNA & $1 \mu \mathrm{l}$ \\
\hline
\end{tabular}


Table 18: Standard PCR protocol for KO clone validation on cDNA level

\begin{tabular}{|l|l|l|}
\hline \multicolumn{1}{|c|}{ Step } & \multicolumn{1}{|c|}{ Temperature $\left({ }^{\circ} \mathrm{C}\right)$} & \multicolumn{1}{c|}{ Time } \\
\hline Denaturation & 95 & $2 \mathrm{~min}$ \\
\hline \multirow{3}{*}{$\begin{array}{l}\text { Amplification } \\
(x 40)\end{array}$} & 94 & $30 \mathrm{sec}$ \\
\cline { 2 - 4 } & 56 & $30 \mathrm{sec}$ \\
\cline { 2 - 4 } & 72 & $30 \mathrm{~min}$ \\
\hline Elongation & 72 & $5 \mathrm{~min}$ \\
\hline
\end{tabular}

\subsubsection{Harvesting of iPSCs and iPSC-CMs}

The iPSC cells were washed once with Versene solution and incubated with Versene for $3 \mathrm{~min}$. The solution was removed, cells were flushed off the plate with PBS and collected into $1.5 \mathrm{ml}$ tubes. Cell samples were pelleted by centrifugation at 13,000 rpm for $1 \mathrm{~min}$. Supernatant was removed and cells subsequently used for DNA isolation or snap frozen in liquid nitrogen. For iPSC-CMs, cells were washed with PBS and harvested using a cell scraper. After transfer into a $1.5 \mathrm{ml}$ tube, the same steps were performed as it was described for the iPSCs.

\subsubsection{Thawing and cultivation of HEK293T cells}

Frozen HEK293T cells were thawed in a water bath at $37{ }^{\circ} \mathrm{C}$ and resuspended in $10 \mathrm{ml} \mathrm{HEK}$ medium (Table 3). After centrifugation for $5 \mathrm{~min}$ at $1000 \mathrm{rpm}$, the supernatant was removed and the cells were resuspended in fresh HEK medium before plating onto uncoated 6 well plates. The cells were kept at $37^{\circ} \mathrm{C}$ and $5 \% \mathrm{CO} 2$.

As soon as the cells reached a confluence of $80-90 \%$, they were washed carefully with PBS. Afterwards the cells were detached using HEK medium and transferred in lower densities onto new 6 well plates. Cells were incubated at $37^{\circ} \mathrm{C}$ with $5 \% \mathrm{CO}_{2}$.

\subsubsection{HEK293T cell transfection}

Transfection of HEK293T cells was performed using Lipofectamine 3000 Transfection Reagent according to the manufacturer's instructions. In brief, the cells were split onto Matrigel coated glass cover slips. The transfection was performed at a confluence of 70-90\%. $3.75 \mu$ and 7.5 $\mu \mathrm{l}$ Lipofectamine 3000 Reagent were each mixed with $125 \mu$ l Opti-MEM Medium. In an additional tube, $250 \mu \mathrm{l}$ Opti-MEM Medium was mixed with $5 \mu \mathrm{g}$ Plasmid-DNA and $10 \mu \mathrm{l}$ P3000 Reagent. $125 \mu$ l of each was mixed and incubated for 5 min at RT before adding to the HEK293T cells. Cells were incubated at $37^{\circ} \mathrm{C}$ and $5 \% \mathrm{CO}_{2}$ for $48 \mathrm{~h}$ days before analysis. 


\subsubsection{Confocal calcium imaging of iPSC-CMs}

Confocal calcium imaging was previously described by Cyganek et al. (Cyganek et al., 2018). In this thesis, iPSC-CMs between day 60 to 80 were used for confocal calcium imaging. 7-14 days before analysis, $1 \times 10^{5}-1,5 \times 10^{5}$ cells were replated on Matrigel coated glass cover slips. The cells were incubated with $5 \mu \mathrm{M}$ Rhod-2 AM fluorescent calcium indicator and $0.02 \%(\mathrm{w} / \mathrm{v})$ Pluronic F-127 in Tyrode's solution (see Table 5) for 30 minutes at RT. Recordings were performed on paced cells in Tyrode's solution at RT which were stimulated at $0.25 \mathrm{~Hz}$ (18-21 $\mathrm{V}, 3 \mathrm{~ms}$ duration with MyoPacer ES) in a recording chamber with platinum electrodes.

For inotropic stimulation the cells were incubated for $10 \mathrm{~min}$ at RT with either $100 \mathrm{nM}$ or 1 $\mu \mathrm{M} \beta$-sympathomimetic isoprenaline hydrochloride before recordings.

For helothermine toxin treatment, the cells were incubated with $0.5 \mu \mathrm{M}, 1 \mu \mathrm{M}, 2.5 \mu \mathrm{M}$ or 5 $\mu \mathrm{M}$ toxin dissolved in Tyrode's solution and subsequently recorded.

The LSM 710 confocal microscopy system with a 63x/1.4 NA oil objective and Zen software was used and images were captured in the line-scan mode (512 pixels, $45 \mu \mathrm{m}, 1057.7 \mathrm{~Hz}$, 20,000 cycles, pinhole $6 \mathrm{AU}$ ). Excitation of Rhod-2 AM was recorded at $561 \mathrm{~nm}$. Analysis was performed by plotting the mean signal intensity as a function of time using ImageJ, polynomial smoothing (6th order, 10 neighbors) using excel and calcium transient analysis using the Peak Analysis Tool in LabChart Pro 8. The settings were as follows: automatic recognition of resting membrane potential, TStart $15 \%$ of height, TRise and TFall between $0 \%$ and $100 \%$ of the peak height. Calcium transient ( $\mathrm{CaT}$ ) decay 50 was defined as the time of the transient amplitude peak maximum until $50 \%$ signal decay. Time to peak (TtP) was defined as the time from the start to the amplitude maximum of the transient. For each analyzed cell, the individual data set represents a mean of 4 CaT recordings. In total, 180-188 cells were measured from three independent differentiations. LabChart Pro, Microsoft Excel and GraphPad Prism were used to analyze and visualize the data. One-way ANOVA with Tukey's multiple comparisons test and two-tailed student's t-test were used to analyze statistics.

\subsection{Molecular analysis}

\subsubsection{Genotyping}

Genotypes of mice were analyzed by PCR using primers from Table 9 and MangoTag Polymerase. Genomic DNA (gDNA) was isolated from either tail biopsies or ear punches which were digested in $200 \mu \mathrm{l}$ DirectPCR tail lysis reagent supplemented with $200 \mu \mathrm{g} / \mathrm{ml}$ Proteinase 
$\mathrm{K}$ at $55^{\circ} \mathrm{C}$ and $300 \mathrm{rpm}$ overnight. Proteinase $\mathrm{K}$ was heat inactivated at $85^{\circ} \mathrm{C}$ for $45 \mathrm{~min}$ prior to the PCR reaction (Table 19 and Table 20).

Table 19: Standard genotyping reaction

\begin{tabular}{|l|l|}
\hline \multicolumn{1}{|c|}{ Reagents } & \multicolumn{1}{c|}{ Volume $(\mu \mathrm{l})$} \\
\hline $5 \times$ reaction buffer & 5.0 \\
\hline $\mathrm{MgCl}_{2}(50 \mathrm{mM})$ & 1.5 \\
\hline $\mathrm{dNTPs}_{(100 \mathrm{mM})}$ & 0.5 \\
\hline Forward primer $(10 \mathrm{pmol} / \mu \mathrm{l})$ & 1.0 \\
\hline Reverse primer $(10 \mathrm{pmol} / \mu \mathrm{l})$ & 1.0 \\
\hline $\mathrm{H}_{2} \mathrm{O}$ & 14.8 \\
\hline MangoTaq Polymerase & 0.2 \\
\hline DNA & 1.0 \\
\hline
\end{tabular}

Table 20: Standard genotyping protocol

\begin{tabular}{|l|l|l|}
\hline \multicolumn{1}{|c|}{ Step } & \multicolumn{1}{|c|}{ Temperature $\left({ }^{\circ} \mathrm{C}\right)$} & \multicolumn{1}{c|}{ Time } \\
\hline Denaturation & 95 & $3 \mathrm{~min}$ \\
\hline \multirow{3}{*}{ Amplification (35x) } & 95 & $30 \mathrm{sec}$ \\
\cline { 2 - 4 } & $57-64$ & $40 \mathrm{sec}$ \\
\cline { 2 - 4 } & 72 & $1 \mathrm{~min}$ \\
\hline Elongation & 72 & $5 \mathrm{~min}$ \\
\hline
\end{tabular}

\subsubsection{DNA isolation}

For DNA isolation of iPSCs or iPSC-CMs the QIAamp DNA Mini kit was used and performed according to manufacturer's instructions. Samples were resuspended in $200 \mu$ l PBS. $20 \mu \mathrm{l}$ Proteinase $\mathrm{K}$ and $200 \mu \mathrm{l}$ Buffer AL was added and samples pulse-vortexed for $15 \mathrm{sec}$. After incubation at $56^{\circ} \mathrm{C}$ for $10 \mathrm{~min}, 200 \mu \mathrm{l} 100 \%$ ethanol was added and samples were again mixed by pulse-vortexing for $15 \mathrm{sec}$. The mixture was loaded onto a spin column and centrifuged for $1 \mathrm{~min}$ at $8,000 \mathrm{rpm}$. Samples were washed first with $500 \mu \mathrm{l}$ Buffer AW1 followed by centrifugation for $1 \mathrm{~min}$ at $8000 \mathrm{rpm}$ and second with $500 \mu \mathrm{l}$ Buffer AW2 followed by centrifugation for $3 \mathrm{~min}$ at full speed. The columns were transferred into a fresh $1.5 \mathrm{ml}$ tube and $30 \mu \mathrm{l}$ nuclease-free water added to the membrane. After incubation at RT for $1 \mathrm{~min}$, the DNA was eluted by centrifugation for $1 \mathrm{~min}$ at $8000 \mathrm{rpm}$. DNA concentration was measured using Nanodrop One. Subsequently, the samples were used for PCR or stored at $-20^{\circ} \mathrm{C}$. 


\subsubsection{RNA isolation}

In order to obtain RNA from heart samples, isolated mouse CMs or iPSC-CMs, the RNeasy Fibrous tissue Mini kit was used according to the manufacturer's instructions.

For snap frozen heart tissues, the samples were placed into a $2 \mathrm{ml}$ tube containing a $7 \mathrm{~mm}$ stainless steel bead. $300 \mu \mathrm{l}$ RLT buffer containing $40 \mathrm{mM}$ DTT was added and the samples were homogenized for $5 \mathrm{~min}$ at $50 \mathrm{~Hz}$ using the Tissue Lyser LT. For the cardiomyocytes, snap frozen pellets were resuspended in $350 \mu \mathrm{l}$ RT buffer containing $40 \mathrm{mM}$ DTT and pulled through a $20 \mathrm{G}$ syringe 6 times to disrupt the cells.

The following steps are the same for all samples. $590 \mu \mathrm{l}$ RNase-free water with $10 \mu$ Proteinase $\mathrm{K}$ was added and the lysates incubated at $55^{\circ} \mathrm{C}$ for $10 \mathrm{~min}$. All samples were centrifuged for 3 $\min$ at $10,000 \times g$ and the supernatant transferred into a new $1.5 \mathrm{ml}$ tube before 0.5 volumes of $100 \%$ ethanol was mixed. The precipitated RNA was transferred into a spin column and centrifuged for $15 \mathrm{sec}$ at $8,000 \times \mathrm{g}$. The samples were washed with $350 \mu \mathrm{l}$ RW1 buffer and centrifuged again for $15 \mathrm{sec}$ at 8,000 x g. The membrane was incubated with $10 \mu \mathrm{l}$ DNase I in $70 \mu \mathrm{l}$ RDD buffer for $15 \mathrm{~min}$ at RT followed by a second wash with buffer RW1. The samples were washed twice with $500 \mu \mathrm{l}$ RPE Buffer and centrifuged first for $15 \mathrm{sec}$ at 8,000 x g, second for $2 \mathrm{~min}$ at $8,000 \times \mathrm{g}$. After centrifugation at full speed for $1 \mathrm{~min}$ to remove all residuals, 30 $\mu \mathrm{l}$ RNAse-free water was added to the membrane and the RNA eluted into a RNAse-free 1.5 $\mathrm{ml}$ tube by centrifuging for $1 \mathrm{~min}$ at $8,000 \times \mathrm{g}$. This step was repeated by using the eluate to increase the yield. RNA concentrations were measured using Nanodrop One. The RNA was subsequently used for reverse transcription or stored at $-80^{\circ} \mathrm{C}$.

\subsection{4 cDNA synthesis}

Total RNA was reverse transcribed into complementary DNA (cDNA) using the RT2 First-strand kit as described in the instructions. $200 \mathrm{ng}-1 \mu \mathrm{g}$ RNA was mixed with $2 \mu \mathrm{l}$ Buffer GE and RNAse-free water to a total volume of $10 \mu \mathrm{l}$ to eliminate genomic DNA. The mixture was incubated for $5 \mathrm{~min}$ at $42^{\circ} \mathrm{C}$ and immediately placed on ice for $1 \mathrm{~min}$. The reverse-transcription mix was prepared using $4 \mu \mathrm{l}$ 5x Buffer BC3, $1 \mu$ C Control P2, $1 \mu$ l RE3 Reverse Transcriptase Mix and $3 \mu \mathrm{l}$ RNase-free water for each sample and added to the genomic DNA elimination mix. Afterwards, the samples were incubated at $37^{\circ} \mathrm{C}$ for $1 \mathrm{~h}$ followed by an incubation at $95^{\circ} \mathrm{C}$ for $5 \mathrm{~min} .91 \mu \mathrm{l}$ RNAse-free water was added to the reaction and the samples were stored at $-20^{\circ} \mathrm{C}$. 
To allow sequencing of murine cDNA samples, total RNA was reverse transcribed using the Superscript IV reverse transcriptase together with Oligo d(T) primers. $200 \mathrm{ng}-1 \mu \mathrm{g}$ RNA was mixed with $1 \mu \mathrm{l} 50 \mu \mathrm{M}$ Oligo $\mathrm{d}(\mathrm{T})$ primers and nuclease-free water to a total volume of $13 \mu \mathrm{l}$. The samples were incubated at $65^{\circ} \mathrm{C}$ for $5 \mathrm{~min}$ followed by incubation on ice for at least $1 \mathrm{~min}$. In between, $4 \mu$ l of $5 x$ SSIV Buffer was mixed with $1 \mu$ I 100 mM DTT and $1 \mu$ I SuperScript IV Reverse Transcriptase and afterwards added to the RNA mix. The samples were incubated at $55^{\circ} \mathrm{C}$ for $10 \mathrm{~min}$ and $80^{\circ} \mathrm{C}$ for $10 \mathrm{~min}$ and stored at $-20^{\circ} \mathrm{C}$ afterwards.

\subsubsection{Real time quantitative PCR}

For RT-qPCR $1 \mu$ l of cDNA was used and mixed according to Table 21 to load onto a 96-well RT-qPCR plate and entered into the BioRad CFX Maestro or according to Table 22 to load onto a 384-well plate and entered into ABI 7900 HT SDS. The reaction was performed at conditions according to Table 23.

Table 21: RT-qPCR reaction mix for a 384-well plate

\begin{tabular}{|l|l|}
\hline \multicolumn{1}{|c|}{ Reagents } & \multicolumn{1}{c|}{ Volume $(\mu \mathrm{ll})$} \\
\hline RT2 SYBR Green Mastermix & 5 \\
\hline Forward Primer $(10 \mu \mathrm{M})$ & 0.5 \\
\hline Reverse Primer $(10 \mu \mathrm{M})$ & 0.5 \\
\hline RNase free water & 2.8 \\
\hline ROX & 0.2 \\
\hline CDNA & 1 \\
\hline
\end{tabular}

Table 22: RT-qPCR reaction mix for a 96-well plate

\begin{tabular}{|l|l|}
\hline \multicolumn{1}{|c|}{ Reagents } & \multicolumn{1}{c|}{ Volume $(\mu \mathrm{l})$} \\
\hline RT2 SYBR Green Mastermix & 12.5 \\
\hline Forward Primer $(10 \mu \mathrm{M})$ & 0.5 \\
\hline Reverse Primer $(10 \mu \mathrm{M})$ & 0.5 \\
\hline RNase free water & 10.5 \\
\hline cDNA & 1 \\
\hline
\end{tabular}

Table 23: RT-qPCR cycling program

\begin{tabular}{|l|l|l|}
\hline \multicolumn{1}{|c|}{ Step } & \multicolumn{1}{|c|}{ Temperature $\left({ }^{\circ} \mathrm{C}\right)$} & \multicolumn{1}{c|}{ Time } \\
\hline Denaturation & 95 & $10 \mathrm{~min}$ \\
\hline \multirow{2}{*}{ Amplification (50x) } & 95 & $15 \mathrm{sec}$ \\
\cline { 2 - 4 } & 60 & $1 \mathrm{~min}$ \\
\hline
\end{tabular}




\begin{tabular}{|l|l|l|}
\hline Melt curve & $55-95$ & $6 \mathrm{sec} / \mathrm{step}$ \\
\hline
\end{tabular}

\subsubsection{Agarose gel electrophoresis}

Gels containing usually $1.5 \%$ of agarose in $1 \times$ TBE buffer (Table 5, stock solution diluted 1:5 in $\mathrm{dd}_{2} \mathrm{O}$ ) were used for separation of DNA fragments. $2.2 \mathrm{~g}$ of agarose was dissolved in $150 \mathrm{ml}$ of 1x TBE buffer and heated until all agarose was dissolved. $6 \mu$ l Midori green was added to the gel after cooling and poured onto a horizontal gel chamber. 1x TBE buffer was used as electrophoresis buffer and electrophoresis carried out at a steady voltage of 100-120 V. The size of the DNA fragments in the agarose gels was determined using GeneRuler $1 \mathrm{~kb}$ DNA ladder. DNA fragments were detected and analyzed by BioDoc Analyze Darkhood.

\subsubsection{DNA isolation from agarose gels}

For gel extraction, the QIAquick Gel Extraction Kit was used according to the manufacturer's instructions. In short, DNA fragments were excised from the gel and dissolved completely in buffer $Q G$ at $50^{\circ} \mathrm{C}$. Isopropanol was added and the mixture loaded onto a spin column. After centrifugation, washing steps using buffer QG and buffer PE were performed before DNA was eluted using $\mathrm{ddH}_{2} \mathrm{O}$. Concentration was measured using NanoDrop One.

\subsection{Protein chemistry}

\subsubsection{Total protein extraction}

For applications like western blot or mass spectrometry for proteome analysis, heart tissue or cells were mechanically homogenized and lysed using RIPA buffer.

In short, the cells were once washed and scratched with $1 \mathrm{ml}$ 1x PBS and transferred into a 1.5 $\mathrm{ml}$ tube. The cells were collected by centrifugation at 13,000 rpm for $1 \mathrm{~min}$. Afterwards, the cell pellet was resuspended in $50 \mu$ I RIPA buffer (Table 5) and incubated for $10 \mathrm{~min}$. The cell suspension was centrifuged at $4{ }^{\circ} \mathrm{C}$ for $5 \mathrm{~min}$ at 5,000 rpm and the supernatant used for analysis. The protein concentration was determined using the BCA Assay as described in the manufacturer's instructions.

For analysis of hearts, $30 \mathrm{mg}$ of snap frozen heart tissue were placed into a $2 \mathrm{ml}$ tube containing a $7 \mathrm{~mm}$ stainless steel bead that was precooled in the freezer. $300 \mu$ I RIPA buffer (Table 5) was added and samples immediately placed into the Tissue Lyser and homogenized for 5 min with $50 \mathrm{~Hz}$. The samples were incubated on ice for $30 \mathrm{~min}$ before centrifugation at 
$16,000 \times \mathrm{g}$ for another $15 \mathrm{~min}$. The supernatant was transferred into a fresh tube and the protein concentration determined using the BCA Assay.

\subsubsection{Immunoblotting}

For protein analysis on western blot, $70 \mu \mathrm{g}$ of protein was mixed with $4 x$ Laemmli and 1:10 2 M DTT and incubated for $5 \mathrm{~min}$ at $95^{\circ} \mathrm{C}$. The samples and the protein size marker were loaded onto a TGX stain-free cast gel in an electrophoresis chamber and run for $45 \mathrm{~min}$ at $200 \mathrm{~V}$ and $20 \mathrm{~mA}$. Proteins were transferred onto a nitrocellulose membrane with TransBlot Turbo for 7 $\min$ at $1.3 \mathrm{~A}$ and $25 \mathrm{~V}$. The membrane was then blocked with blocking buffer (Table 5) for $1 \mathrm{~h}$ at RT. The primary antibody was diluted in $1 \%$ milk or $1 \%$ BSA in TBST according to Table 7 and the membrane was incubated over night at $4{ }^{\circ} \mathrm{C}$. On the following day, the membrane was washed $2 x$ for 5 min and $1 x$ for 15 min in TBST before adding the secondary HRP antibody diluted $1: 10,000$ in $1 \%$ milk or $1 \%$ BSA in TBST for $1 \mathrm{~h}$ at RT. The membrane was washed $3 \mathrm{x}$ for $5 \mathrm{~min}$ and $1 \mathrm{x}$ for $30 \mathrm{~min}$ with TBST and the protein bands were visualized using SuperSignal West Femto Kit with a Chemidoc XRS+ imager.

\subsection{Histological and immunocytochemical analyses}

\subsubsection{Immunocytochemistry of iPSC-CMs}

For iPSC-CMs, immunocytochemistry was performed as previously described (Kleinsorge \& Cyganek, 2020). The cells were replated in lower density on matrigel-coated glass cover slips. Following 7 days of recovery, cells were washed 3x with PBS before fixing with $4 \%$ Roti-Histofix for $20 \mathrm{~min}$. After 3 additional washing steps, the cells were incubated in staining buffer (Table 5 ) at $4{ }^{\circ} \mathrm{C}$ until needed. The primary antibody was diluted in cell permeabilization buffer for cell permeabilization and the cells were incubated over night at $4{ }^{\circ} \mathrm{C}$. On the following day, the secondary antibody was diluted in staining buffer and the samples were incubated for $1 \mathrm{~h}$ at RT. After the first washing step with PBS, the cells were incubated with Hoechst33342 diluted 1:1,000 in PBS for 10 min at RT. Subsequently, the cells were washed $3 x$ for 5 min with PBS and one time for 10 min with $\mathrm{dd}_{2} \mathrm{O}$. Glass cover slips were mounted with Fluoromount$\mathrm{G}$ on microscope slides. The image recording was performed using the Zeiss LSM 710 confocal microscopy system and Zen software. The images were analyzed using ImageJ.

For immunocytochemical staining of HEK293T cells, the cells were used $48 \mathrm{~h}$ after transfection. After washing $2 x$ with PBS, the cells were fixed with $4 \%$ Roti-Histofix for 10 min at RT and 
subsequently permeabilized with cell permeabilization buffer for $15 \mathrm{~min}$ at RT. Cells were blocked in $2 \%$ BSA in PBS for $1 \mathrm{~h}$ and incubated at $4{ }^{\circ} \mathrm{C}$ over night with the primary antibody which was diluted in $2 \%$ BSA in PBS. After washing $3 \times 5$ min with PBS, cells were incubated 1 $h$ at RT with the secondary antibody which was diluted in $2 \%$ BSA in PBS. HEK293T cells were washed $3 \times 5$ min with PBS and incubated with Hoechst33342 diluted 1:1,000 in PBS for 10 min at RT. After 3 additional washing steps for $5 \mathrm{~min}$, the glass cover slips were mounted with Fluoromount-G on microscope slides. The image recording was performed using Zeiss LSM 710 confocal microscopy system with a 63x/1.4 NA oil objective and Zen software.

\subsubsection{Langendorff CM isolation}

Mouse cardiomyocytes were isolated using a Langendorff perfusion system as described before (Dybkova et al., 2018). Mice were anesthetized and sacrificed by cervical dislocation. The heart was dissected and transferred into PBS. The heart was connected to a 20G cannula, fixed and transferred onto the Langendorff apparatus. The heart was retrogradely perfused with first Tyrode's solution (1x) (Table 5) and continued with Tyrode's solution supplemented with Liberase TM, $2.5 \%$ trypsin and $10 \mathrm{mM} \mathrm{CaCl}_{2}$ at a flow rate of $3.5 \mathrm{ml} / \mathrm{min}$ for $9 \mathrm{~min}$ at 37 ${ }^{\circ} \mathrm{C}$ in total. Afterwards, the atria were carefully removed and the digested ventricles dissected and homogenized by using a pasteur pipette in $2.5 \mathrm{ml}$ Tyrode's solution supplemented with $2.5 \mathrm{ml}$ stop solution (Table 5). The cells were transferred into a falcon through a strainer. After 7 min of sedimentation, the supernatant was removed or pelleted for non-cardiomyocyte downstream application. The remaining cardiomyocytes were either used for calcium recordings or washed with PBS and pelleted for $1 \mathrm{~min}$ at 13,000 rpm. Pellets were snap frozen in liquid nitrogen and stored at $-80^{\circ} \mathrm{C}$ for RNA isolation.

\subsubsection{Paraffin embedding, dewaxing and rehydration}

Isolated hearts were fixed in Histofix at $4{ }^{\circ} \mathrm{C}$ over night. The fixed hearts were dehydrated in an ethanol series and embedded in paraffin using a tissue processor. Therefore, hearts were incubated in $60 \%$ ethanol, 2x $75 \%$ ethanol, 2x $96 \%$ ethanol, 2x $100 \%$ ethanol, 2x xylol, and $3 x$ paraffin, each for 90 min followed by embedding into paraffin blocks. $5 \mu \mathrm{m}$ thick sections were dissected using a microtome. For downstream methods, sections were dewaxed twice in xylol for $5 \mathrm{~min}$, followed by rehydration in an ethanol series: 2x $100 \%, 95 \%, 70 \%, 50 \%, 30$ $\%$, and distilled water, each step for $5 \mathrm{~min}$. 


\subsubsection{Picro Sirius Red Staining}

Dewaxed and fixed heart sections were stained using the Picro Sirius Red Stain Kit following manufacturer's instructions. Briefly, the heart tissue sections were incubated with Picro Sirius Red solution for 60 min at RT followed by rinsing twice in acetic acid solution and once in 100 $\%$ ethanol. Slides were cleared and mounted in Permount Medium.

\subsubsection{Wheat germ Agglutinin staining}

In order to measure the minimal fiber diameter (MFD), cell borders of heart sections were visualized using WGA. Dewaxed and rehydrated sections were washed twice with Hank's balanced salt solution (HBSS) without phenol and incubated with WGA488 conjugate in HBSS at a final concentration of $10 \mu \mathrm{g} / \mathrm{ml}$. Staining is followed by two washing steps with PBS. The heart sections were mounted using ProLong Gold antifade supplemented with DAPI and stored at a dark place.

\subsubsection{Quantification of histological stainings}

Histological heart sections were analyzed using ImageJ distribution (Schindelin et al., 2012; Rueden et al., 2017). For MFD analysis one heart section per mouse was captured completely using 10x magnification. The images of the heart were saved in .tiff format and were then converted to 8-bit tiff and analyzed using semi-automated segmentation. Briefly, the images were transformed to greyscale images. The contrast of these greyscale images was enhanced and the background subtracted followed by conversion into binary images to identify and analyze particles. The resulting data were visualized and statistically analyzed using GraphPad Prism and one-way ANOVA, respectively.

To quantify fibrotic areas in the mouse heart tissue from Picro Sirius Red staining, images were taken at 10x magnification and analyzed with ImageJ. In short, red-blue-green (RGB) channel images were split and fibrotic regions as well as whole heart section areas measured using automated thresholds. Analysis was performed with Excel and GraphPad Prism using one-way ANOVA.

\subsubsection{Epifluorescence calcium-transient measurement of isolated mouse cardiomyocytes}

Mouse cardiomyocytes were isolated as described above (see 2.15.2) and transients measured with minor changes as previously described (Bengel et al., 2020). Briefly, the cells were reloaded with calcium in increasing concentrations $(0.1 \mathrm{mmol} / \mathrm{l}$ to $0.8 \mathrm{mmol} / \mathrm{l})$ stepwise, 
each for $7 \mathrm{~min}$. Subsequently, the cells were plated onto laminin-coated chambers and settled for 15 min before incubating with $10 \mu \mathrm{M}$ Fura-2 AM and Pluronic F-127 diluted in epifluorescence tyrode (Table 5) for another $15 \mathrm{~min}$. For recordings, this solution was changed to epifluorescence tyrode. The cells were paced with $1 \mathrm{~Hz}$ and $20 \mathrm{~V}$ until they reached a steady state. They were then stimulated with $2 \mathrm{~Hz}$ and $4 \mathrm{~Hz}$ frequencies. The measurements were performed at RT using a Motic AE31 microscope with a fluorescence detection system. Excitation of Fura-2 AM was recorded at $510 \mathrm{~nm}$. The transients were finally analyzed using IONWizard.

\subsection{6 mRNA sequencing}

mRNA sequencing was performed at the Transcriptome and Genome Analysis Laboratory (TAL) core facility in Göttingen according to Khadjeh et. al with some adaptations (Khadjeh et al., 2020). For analysis, 500 ng total RNA was used and the library prepared as described in the manufacturer's instructions from the TruSeq RNA Sample Preparation V2 Kit. Quantifluor dsDNA System was used for quantitation. The DNA 1000 chip served for determinating final cDNA libraries on the Bioanalyzer 2100. These cDNA libraries were amplified and sequenced using the cBot and HiSeq2000 before transformation into bcl files with the BaseCaller Software to demultiplex to fastq files with CASAVA v1.8.2. Quality was controlled via fastqc. The sequence of Homo sapiens (GRCh38/hg38) served as reference for alignment and was performed with the STAR alignment software one allowing for two mismatches within 50 bases. Afterwards, resulting SAM files were converted into sorted BAM files, filtered for unique hits and counted with SAMtools and HTSeq. Final data was preprocessed and analyzed with R/Bioconductor using the DESeq2 package. The data was normalized and tested for differentially expressed genes with a generalized linear model likelihood ratio test assuming negative binomial data distribution. To identify final candidate genes, the genes were filtered to a minimum of $\log 2 \mathrm{FC}>1 /-1$ and a false discovery rate-corrected $p$-value $<0.05$. Annotation of resulting genes was done by using Homo sapiens entries from Ensembl (www.ensembl.org) via the biomaRt package.

Pathway analysis was performed using Cytoscape with the ClueGO plugin (Shannon et al., 2003; Bindea et al., 2009) and KEGG mapper on significantly regulated genes (cut off $\log 2 \mathrm{FC}>0.5 /-0.5$; adjusted $\mathrm{p}$ value $<0.05)$. 


\subsection{Proteome analysis}

Proteome analysis was performed as described in detail before (Khadjeh et al., 2020). 


\section{Results}

\subsection{Characterization of CRISPLD1 in iPSC-CMs}

\subsubsection{Generation and validation of CRISPLD1 KO in iPSCs}

In order to analyze the effects of CRISPLD1 loss in iPSC-CMs, mutations were introduced via CRISPR/Cas9 into an already established WT iPSC line (see Table 1). This WT cell line served as an isogenic control for the experiments. Exon 7 was selected as the target for genome editing to cover all CRISPLD1 isoforms (Figure 3-1A).

CRISPR/Cas9-mediated genome editing led to a deletion of $34 \mathrm{bp}$ in exon 7 in the DNA. This deletion resulted in an in-frame deletion of $303 \mathrm{bp}$ on mRNA level and therefore skipping of the exons 6-8 (Figure 3-1B). As a consequence, 101 aa are lost, affecting all three domains including a V5/Tpx-1 site (Figure 3-1C). This KO cell line was further validated via RT-qPCR, showing that primers that target the deleted region are not able to amplify any product, while this was possible in the WT cell line (Figure 3-1D). When using primer pairs spanning regions outside this deletion, there is still mRNA expression detectable but significantly lower compared to WT controls (Figure 3-1E). Additionally, this mRNA lacks parts of the CAP, V5/Tpx1 and LCCL domains and therefore, I assume a functional KO of CRISPLD1 protein.

However, due to the lack of a functional antibody, it was not possible to validate CRISPLD1 KO on protein level in western blot. In total, four commercially available CRISPLD1 antibodies were tested in WT and KO iPSCS and iPSC-CMs, CRISPLD1 overexpressing HEK293T cells as well as WT HEK293T cells and a commercial CRISPLD1 overexpressing lysate. All antibodies failed to detect endogenous CRISPLD1 specifically (Appendix, Figure 5-1). 


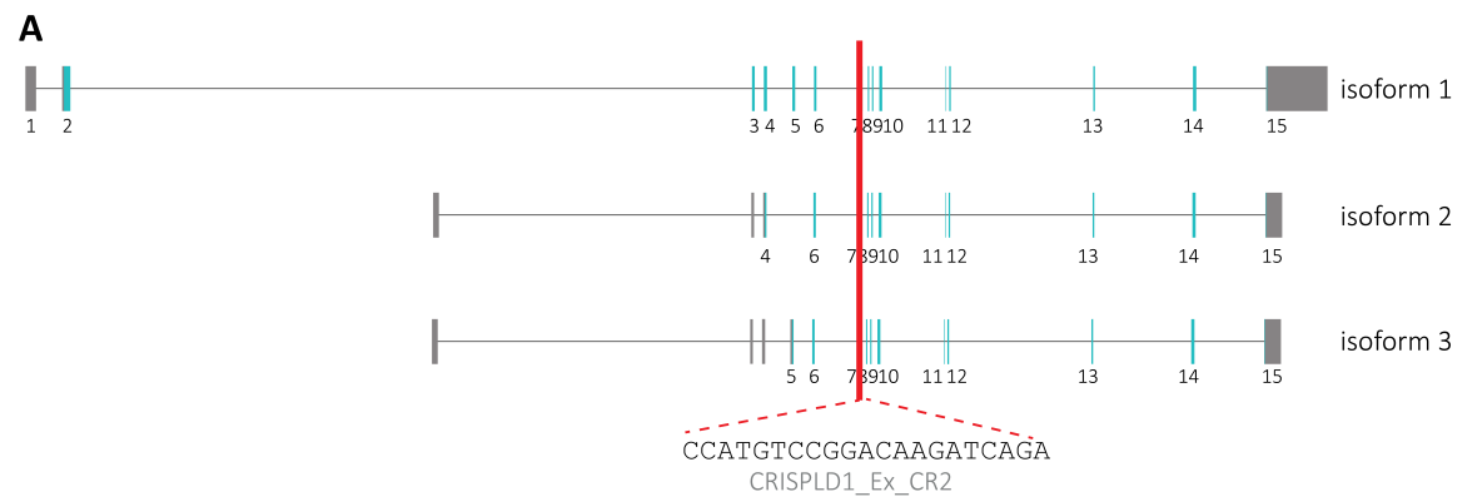

B
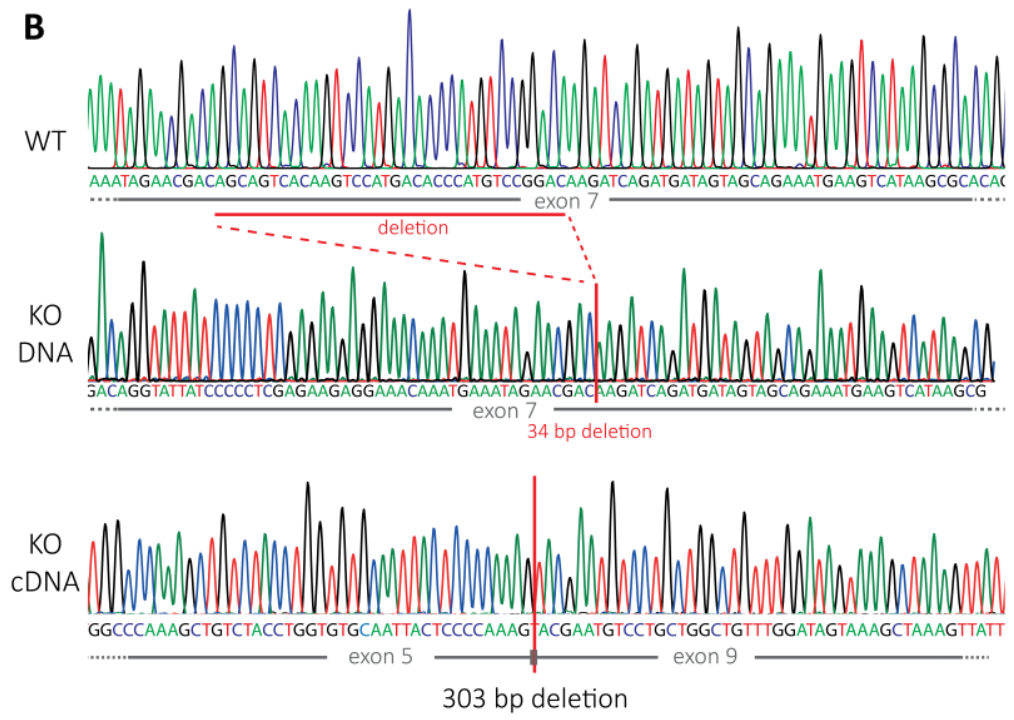

$\mathbf{E}$

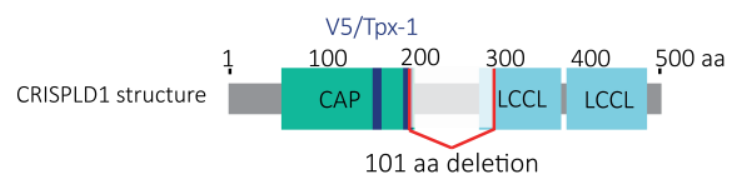

C
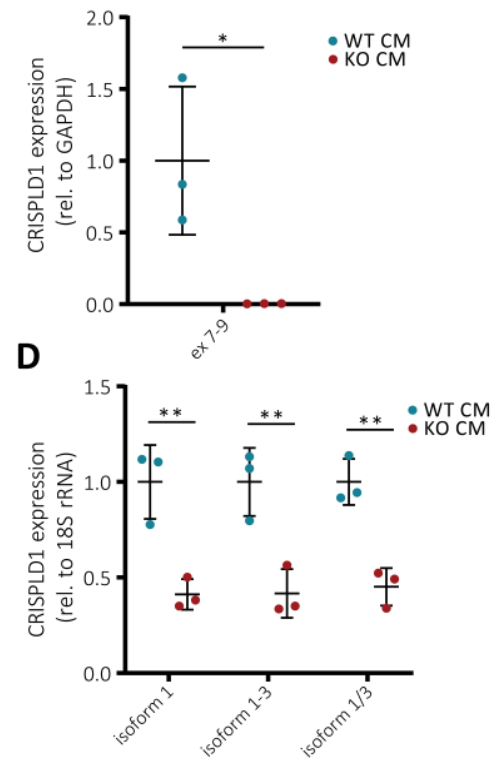

Figure 3-1 Generation and validation of CRISPLD1 KO. (A) The guide RNA (CRISPLD1_Ex_CR2) targets CRISPLD1 exon 7 to cover all isoforms. (B) Sanger sequencing results of the final KO clone compared to WT confirmed a 34 bp deletion of exon 7 on DNA level, and a 303 bp deletion (exon 6-8) on mRNA level. (C) RT-qPCR analysis of the deleted region confirmed that there is no CRISPLD1 expression in KO CMs when compared to WT controls. (D) A significant reduction of CRISPLD1 mRNA of all isoforms could be detected when primers align outside the deleted region. Three independent differentiations/groups were used. Statistics were performed with an unpaired twotailed t-test. Error bars indicate SD, ${ }^{*}=p<0.05, * *=p<0.01$. (E) The deletion of 101 aa affects the functional domains of the protein. Adapted and modified from (Khadjeh et al., 2020).

\subsubsection{CRISPLD1 KO does not alter sarcomeric structure of iPSC-CMs}

CRISPLD1 KO iPSCs and their isogenic controls were directly differentiated into ventricular-like iPSC-CMs as described in 2.12.2. First spontaneous contractions were visible approximately two weeks after differentiation initiation and therefore confirmed an already functional contractile system. Cells were cultured for at least 60 days ( $\mathrm{d} 0=$ start of differentiation) before being used for experiments, if not stated otherwise. 
In order to validate successful differentiation of iPSCs into iPSC-CMs and normal cardiac marker expression, immunofluorescence analysis was performed in WT iPSC-CMs to compare to CRISPLD1 KO iPSC-CMs (Figure 3-2). All CMs show a normal organized sarcomeric structure visualized by $\alpha$-actinin (ACTN) and cardiac troponin T (CTNT). Ventricular identity was confirmed by immunostaining with myosin light chain 2 (MLC2V). Furthermore, no alterations in the expression pattern of the cardiac-specific gap junction protein connexin 43 (CX43) or the ryanodine receptor 2 (RYR2) were obtained.
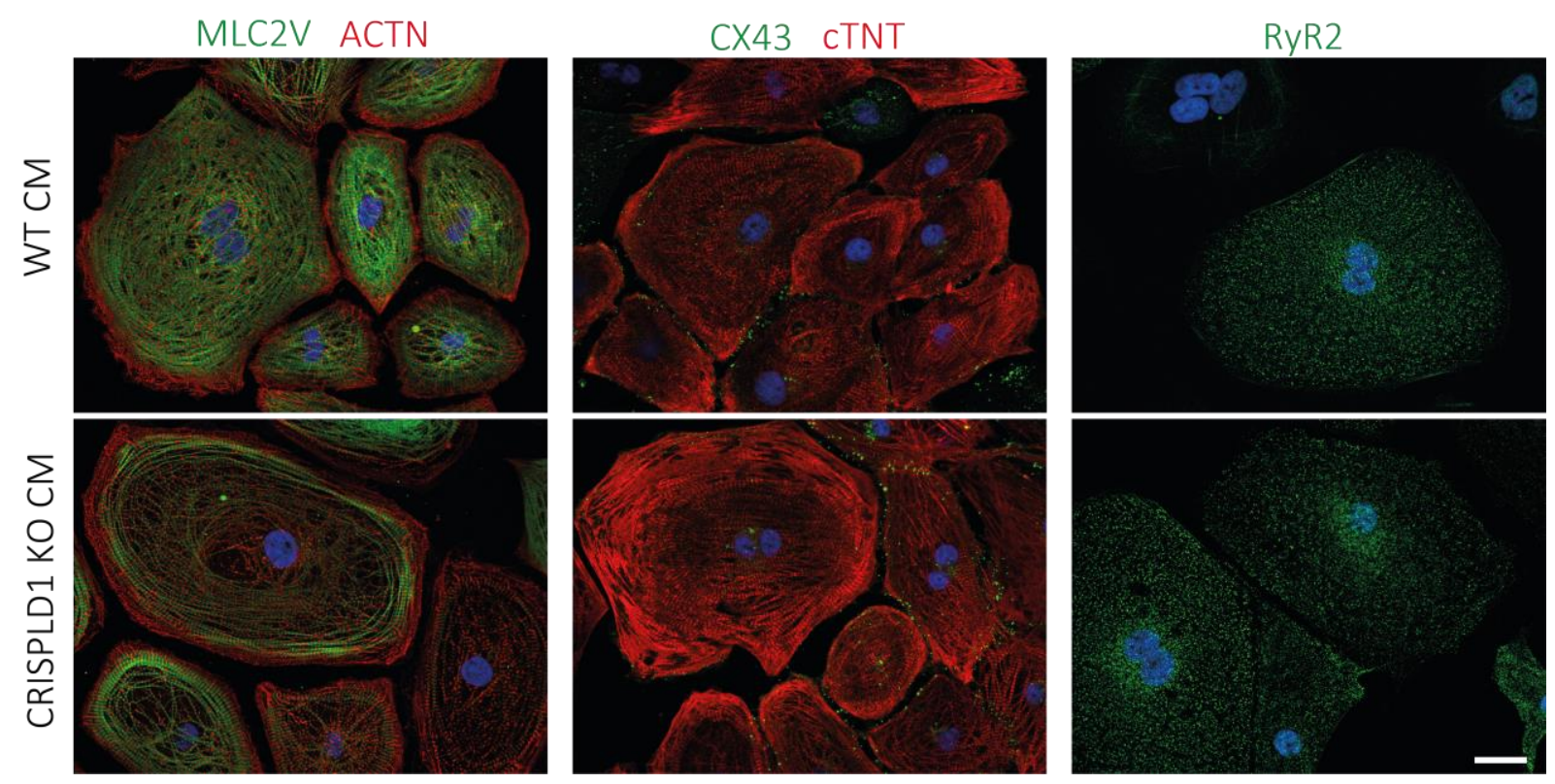

Figure 3-2 CRISPLD1 KO iPSC-CMs show normal cardiac marker expression in IF analysis. Representative images of ventricular differentiated WT and CRISPLD1 KO iPSC-CMs. Fixed cells were stained for myosin light chain 2 (MLC2V; green), $\alpha$-actinin (ACTN; red), connexin-43 (CX43; green), cardiac troponin T (cTNT; red) and ryanodine receptor 2 (RYR2; green). Both cell lines display a characteristic sarcomeric structure as well as normal cardiac marker expression. Nuclei were stained with Hoechst33342 (blue). Scale bar $=20 \mu \mathrm{m}$.

\subsubsection{Calcium cycling of iPSC-CMs is affected by CRISPLD1 KO}

A normal calcium homeostasis is crucial for normal heart function and dysregulation can result in heart diseases such as hypertrophy or heart failure (Frey et al., 2000). Therefore, functional analysis of calcium transients was performed in iPSC-derived CRISPLD1 KO CMs and compared to their isogenic controls. The calcium cycling of iPSC-CMs was assessed between day 63 and 74 to ensure adequate maturation. CRISPLD1 KO iPSC-CMs showed a significant increase in the calcium transient $(\mathrm{CaT})$ amplitude $(\mathrm{WT}$ mean $=2.85$, $\mathrm{KO}$ mean $=3.46$; Figure 3-3A), a significant increase in the CaT rise time (WT mean =169.46, $\mathrm{KO}$ mean $=199.02$; Figure 3-3B) and a significant decrease in CaT decay 50 (WT mean =603.51, KO mean =460.76; Figure 3-3C) pointing towards a shortened, but elevated systolic CaT (Figure 3-3D). 
A

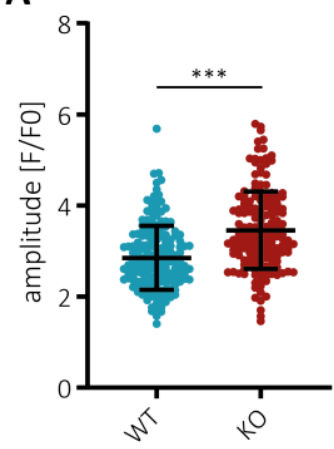

B

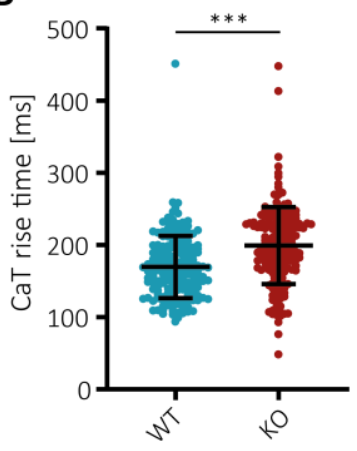

C

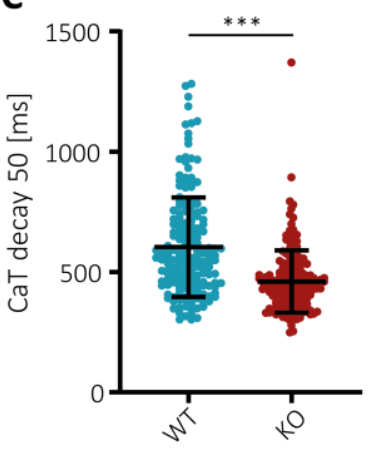

D

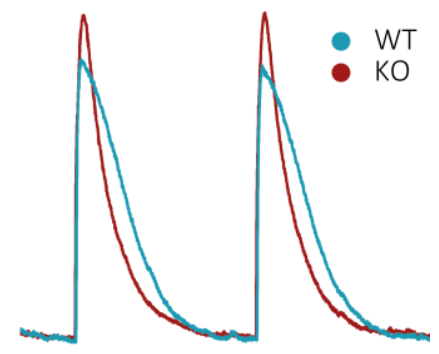

Figure 3-3 Functional analysis of calcium transients of CRISPLD1 KO iPSC-CMs and WT iPSC-CMs at day 63-74 by confocal calcium imaging. Analysis of (A) CaT amplitude and (B) CaT rise time indicated a significant increase in CRISPLD1 KO iPSC-CMs, whereas analysis of (C) CaT decay 50 showed a significant decrease in CRISPLD1 KO iPSC-CMs. (D) Overlay of representative WT (blue) and CRISPLD1 KO (dark red) CaT. Error bars indicate SD, $* * *=$ $p<0.001$. Each point represents measurement for one cell, $n=180-183$.

\subsubsection{CRISPLD1 KO can rescue disturbance of helothermine treated iPSC-CM calcium} transients

In order to validate these findings and to test the influence of a known calcium regulator that is evolutionary related to CRISPLD1, iPSC-CMs were treated with the toxin helothermine. To determine a suitable concentration to analyze the toxin effect on the cells, four different concentrations were tested on WT iPSC-CMs (Figure 3-4).

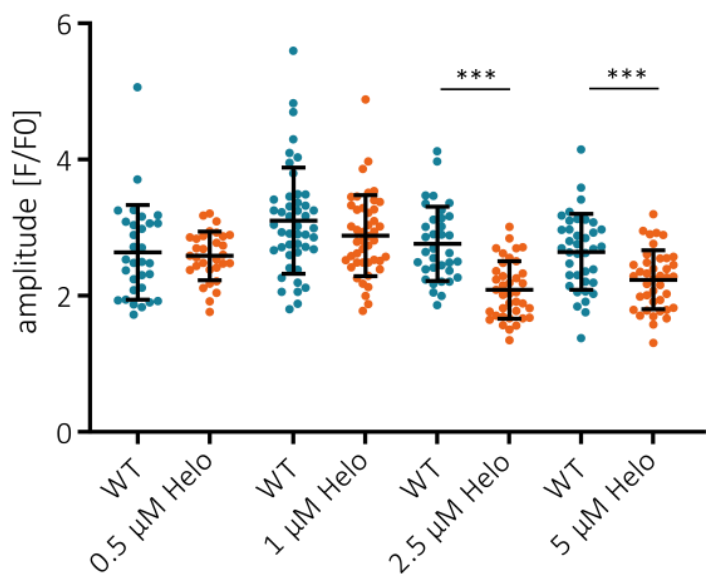

Figure 3-4 Concentration-dependent effect of helothermine treatment on WT IPSC-CM calcium transient amplitude. The CaT amplitude was significantly decreased after $2.5 \mu \mathrm{M}$ and $5 \mu \mathrm{M}$ helothermine treatment. Error bars indicate $S D,{ }^{* *}=p<0.001$. Each point represents measurement for one cell, $n=31-46$. Helo $=$ helothermine.

A significant decrease in CaT amplitude could be seen after treatment with $2.5 \mu \mathrm{M}$ and $5 \mu \mathrm{M}$ helothermine, but no significant reduction when treated with $0.5 \mu \mathrm{M}$ or $1 \mu \mathrm{M}$ helothermine. Therefore, a concentration of $5 \mu \mathrm{M}$ helothermine was selected for analysis of CaT in WT iPSCCMs and CRISPLD1 KO iPSC-CMs. 
A

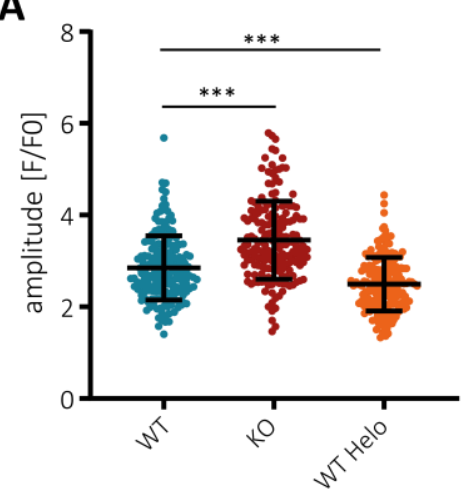

D

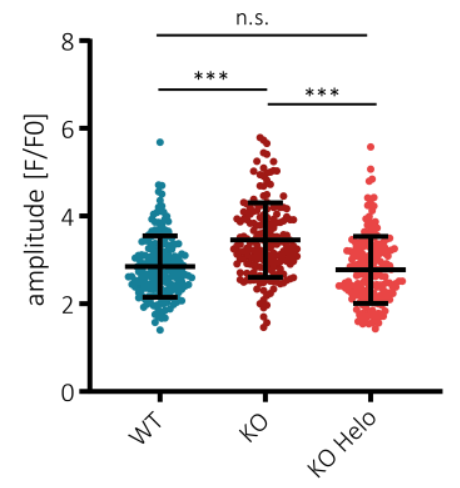

G

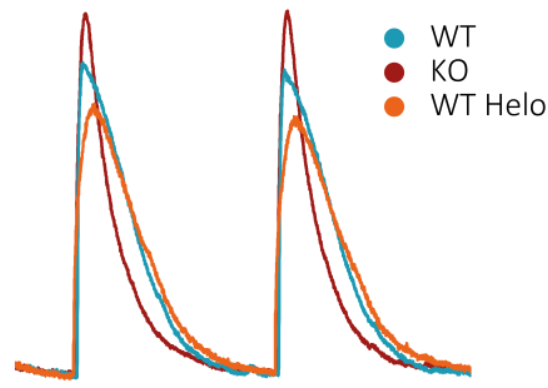

B

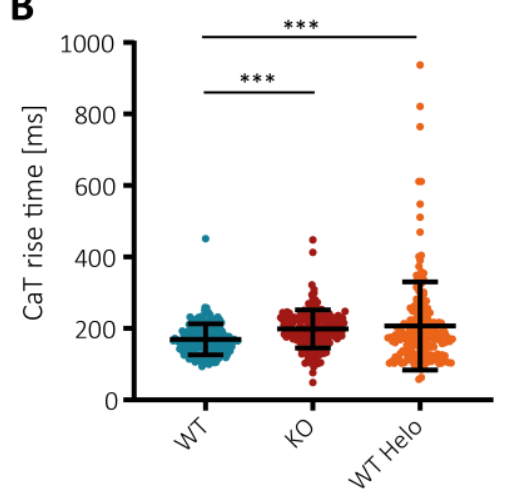

$\mathbf{E}$

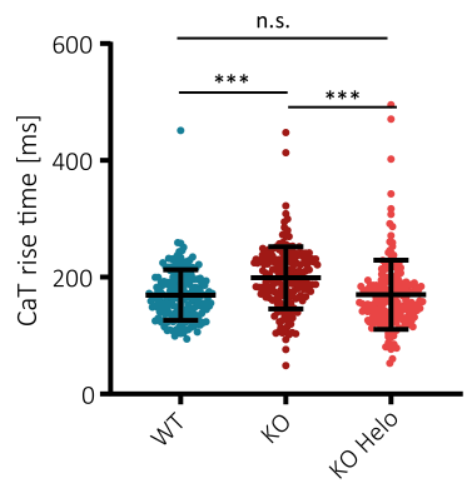

C

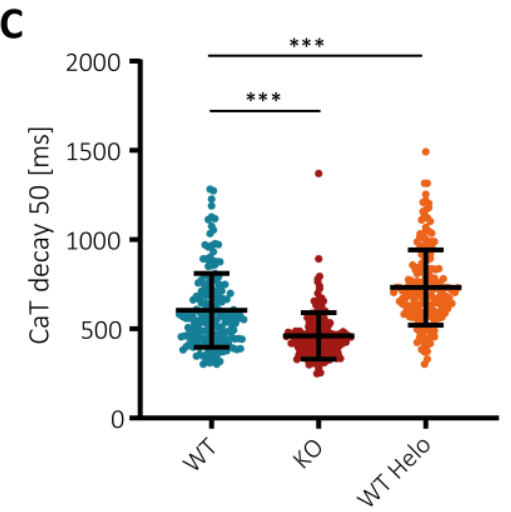

$\mathbf{F}$

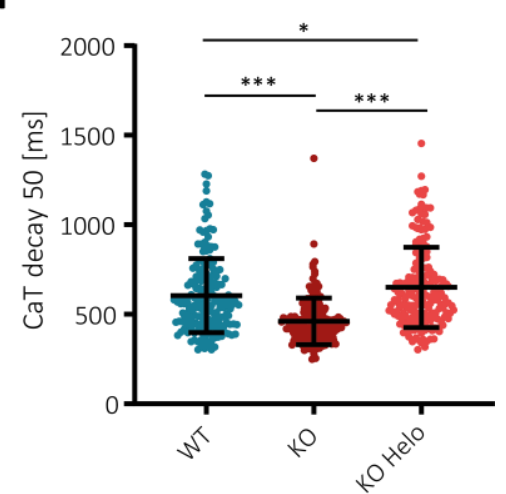

H

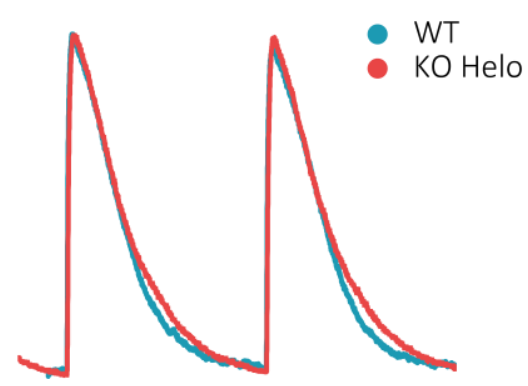

Figure 3-5 Functional analysis of calcium transients of helothermine treated CRISPLD1 KO iPSC-CMs and WT iPSC-CMs at day 63-74 by confocal calcium imaging. Analysis of (A) CaT amplitude (B) CaT rise time and (C) CaT decay 50 in untreated WT and CRISPLD1 KO iPSC-CMs, and helothermine treated WT iPSC-CMs. (D) CaT amplitude, (E) CaT rise time and (F) CaT decay 50 analysis in untreated WT, CRISPLD1 KO IPSC-CMs, and helothermine treated CRISPLD1 KO iPSC-CMs. (G) Overlay of representative WT (blue), CRISPLD1 KO (dark red) and helothermine treated WT (orange) CaT. (H) Overlay of representative WT (blue) and helothermine treated CRISPLD1 KO (light red) CaT. Error bars indicate SD, ${ }^{*}=p<0.05 ;{ }^{* * *}=p<0.001 ; n . s .=$ not significant. Each point represents measurement for one cell, $n=180-188$. Helo $=$ helothermine. Adapted and modified from (Khadjeh et al., 2020).

Helothermine treated WT iPSC-CMs showed a significant decrease in CaT amplitude compared to WT controls without toxin treatment $(\mathrm{WT}$ mean $=2.85, \mathrm{KO}$ mean $=3.46, \mathrm{WT} H \mathrm{HT}$ mean $=$ 2.49; Figure 3-5A). The CaT rise time was significantly increased upon helothermine treatment in WT iPSC-CMs compared to untreated WT iPSC-CMs (WT mean $=169.46$, $\mathrm{KO}$ mean $=199.02$, WT HT mean = 207.04; Figure 3-5B), as well as the CaT decay 50 (WT mean $=603.51$, KO mean $=460.76, \mathrm{WT} H \mathrm{HT}=731.86$; Figure 3-5C). When CRISPLD1 KO iPSC-CMs were treated with 
helothermine, they showed a significant decrease compared to untreated CRISPLD1 KO iPSCCMs, but no significant alteration compared to untreated WT iPSC-CMs (WT mean $=2.85, \mathrm{KO}$ mean = 3.46, $\mathrm{KO} \mathrm{HT}$ mean = 2.77; Figure 3-5D). This was also true for the CaT rise time. The significant increase that was detected in helothermine treated WT iPSC-CMs compared to untreated WT iPSC-CMs was not detectable when comparing helothermine treated CRISPLD1 KO iPSC-CMs to untreated WT iPSC-CMs. Ranges were rather comparable (WT mean = 169.46, KO mean = 199.02, KO HT mean = 170.06; Figure 3-5E). Lastly, the increased CaT decay 50 that was seen in helothermine treated WT iPSC-CMs compared to untreated WT iPSC-CMs was still detectable in helothermine treated CRISPLD1 KO iPSC-CMs. However, this increase was to a much lower extend $(\mathrm{WT}$ mean $=603.51, \mathrm{KO}$ mean $=460.76, \mathrm{KO} \mathrm{HT}=650.14$; Figure 3-5F).

In summary, CRISPLD1 KO had a measurable effect on CaT of iPSC-CMs and showed an increased CaT amplitude and rise time, and decreased CaT decay 50. This CRISPLD1 KO was further able to rescue the effect produced in WT iPSC-CMs after treatment with the calcium regulating toxin helothermine and normalized the CaT amplitude and CaT rise time. These results suggest an inhibitory role of CRISPLD1 in CM calcium homeostasis.

\subsubsection{CRISPLD1 is involved in the regulation of cardiomyopathy, inflammation, calcium regulation and ECM organization}

To identify the molecular pathways that are influenced by the CRISPLD1 KO in iPSC-CMs, samples from four cell differentiations, using three replicates each, were used for RNA sequencing at d60. 3583 differentially expressed genes (DEGs) were identified, from which 73 $\%$ were downregulated and $27 \%$ upregulated after CRISPLD1 KO (Figure 3-6). The downregulated genes showed the most significant enrichment for gene ontology (GO) terms associated with apoptosis signaling, transforming growth factor beta (TGF-beta) signaling, tumor necrosis factor (TNF) signaling, transcriptional regulation by tumor protein p53 (TP53) and WNT signaling pathways. Pathway analysis of the upregulated genes showed an enrichment of GO terms like voltage-gated sodium channel activity, ion transmembrane transporter activity and inorganic cation transmembrane transporter activity which cover 76 $\%$ of the upregulated genes. These identified pathways relate to the results observed in CaT after CRISPLD1 KO. 


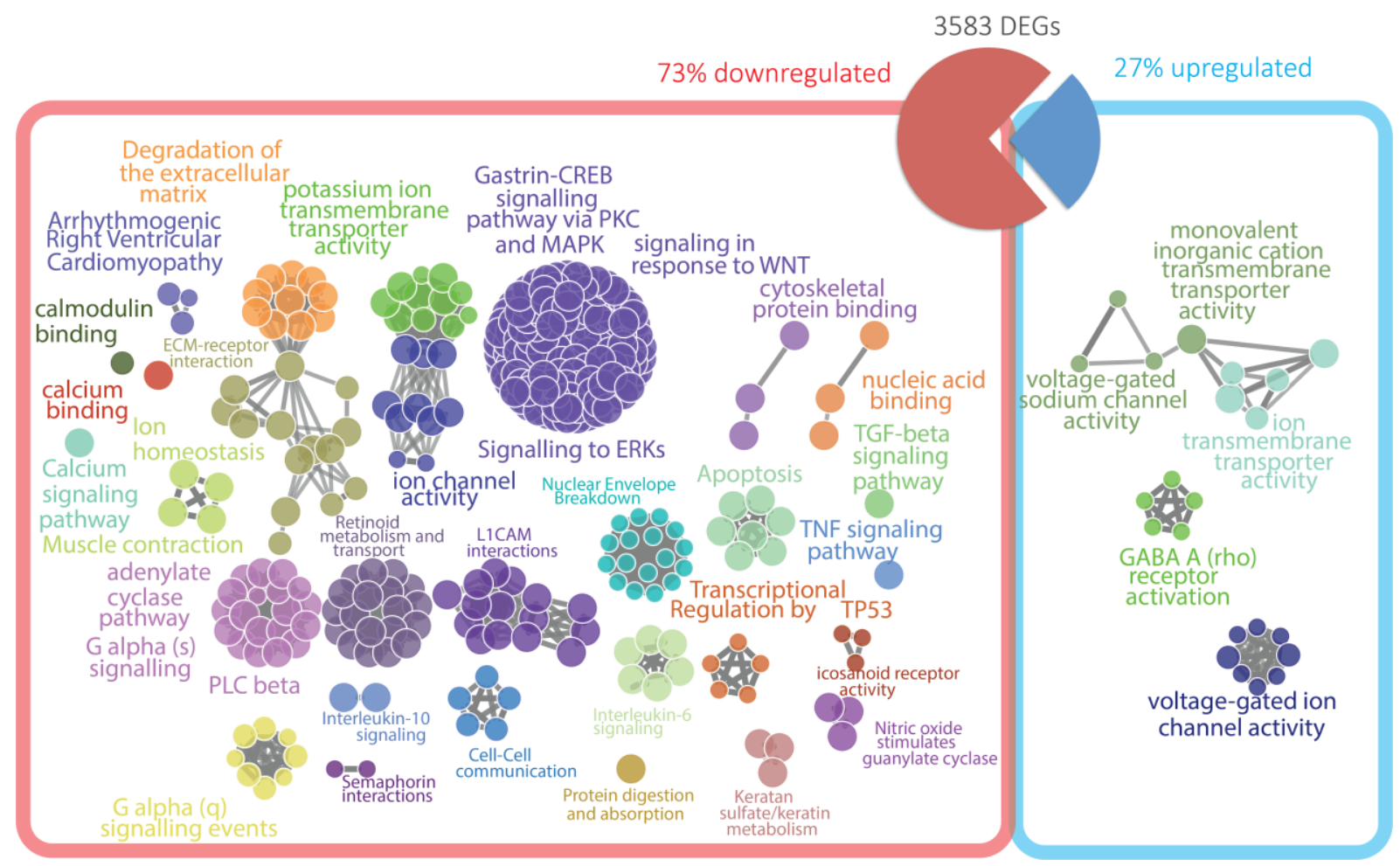

Figure 3-6 ClueGO analysis of transcriptomics of CRISPLD1 KO iPSC-CMs vs. WT iPSC-CMs. RNA-seq revealed 3583 differentially expressed genes comparing WT and CRISPLD1 KO iPSC-CMs (log2 Fold Change $>0.5 /<-0.5$, adjusted p-value $<0.05$ ). $73 \%$ of these genes were found to be downregulated (red), $27 \%$ upregulated (blue). Analysis of the downregulated genes showed significant GO term enrichment for apoptosis signaling, TGF-beta signaling, TNF signaling, transcriptional regulation by TP53 and WNT signaling pathways. The GO terms voltagegated sodium channel activity, ion transmembrane transporter activity and inorganic cation transmembrane transporter activity were enriched in the upregulated genes. The node size represents the significance of GO term enrichment. The different groups are colored and named by their leading term. The illustration was created by S. Khadjeh and modified (Khadjeh et al., 2020).

To validate and visualize gene expression changes, DEGs were mapped to the KEGG adrenergic signaling pathway as this pathway is a key player in cardiac hypertrophy and its transition to HF (Figure 3-7A). A detailed pathway analysis after CRISPLD1 KO in iPSC-CMs showed a significant downregulation of the $\beta-A R$ signaling pathways (ADRB1) and their downstream targets (GNAI2, PIK3R6, AKT3, ADCY3/7, RAPGEF3, MAPK11, CREB3L1/2/3). The angiotensin II receptor type 1, sarcomere genes (MYH6, TPM3/4) and protein phosphatase encoding genes (PPP2R1B, PPP2R2B) are downregulated as well as genes encoding the L-type sodium channel (CACNA1S, CACNA2D3, CACNB3, CACNB4, CACNG4), potassium channel (KCNE1) and the sodium-potassium ATPase (ATP1A2). Among upregulated genes are RYR2, ADRA1B, CAMK2A, MYH7 and genes encoding the cardiac sodium channel (SCN4B, SCN5A). Validation of representative DEG by RT-qPCR showed a significant upregulation of RYR2 and SCN5A in CRISPLD1 KO iPSC-CMs, whereas CACNAG4, ATP1A2, AGTR1 and CACNA2D3 were significantly downregulated (Figure 3-7B). 


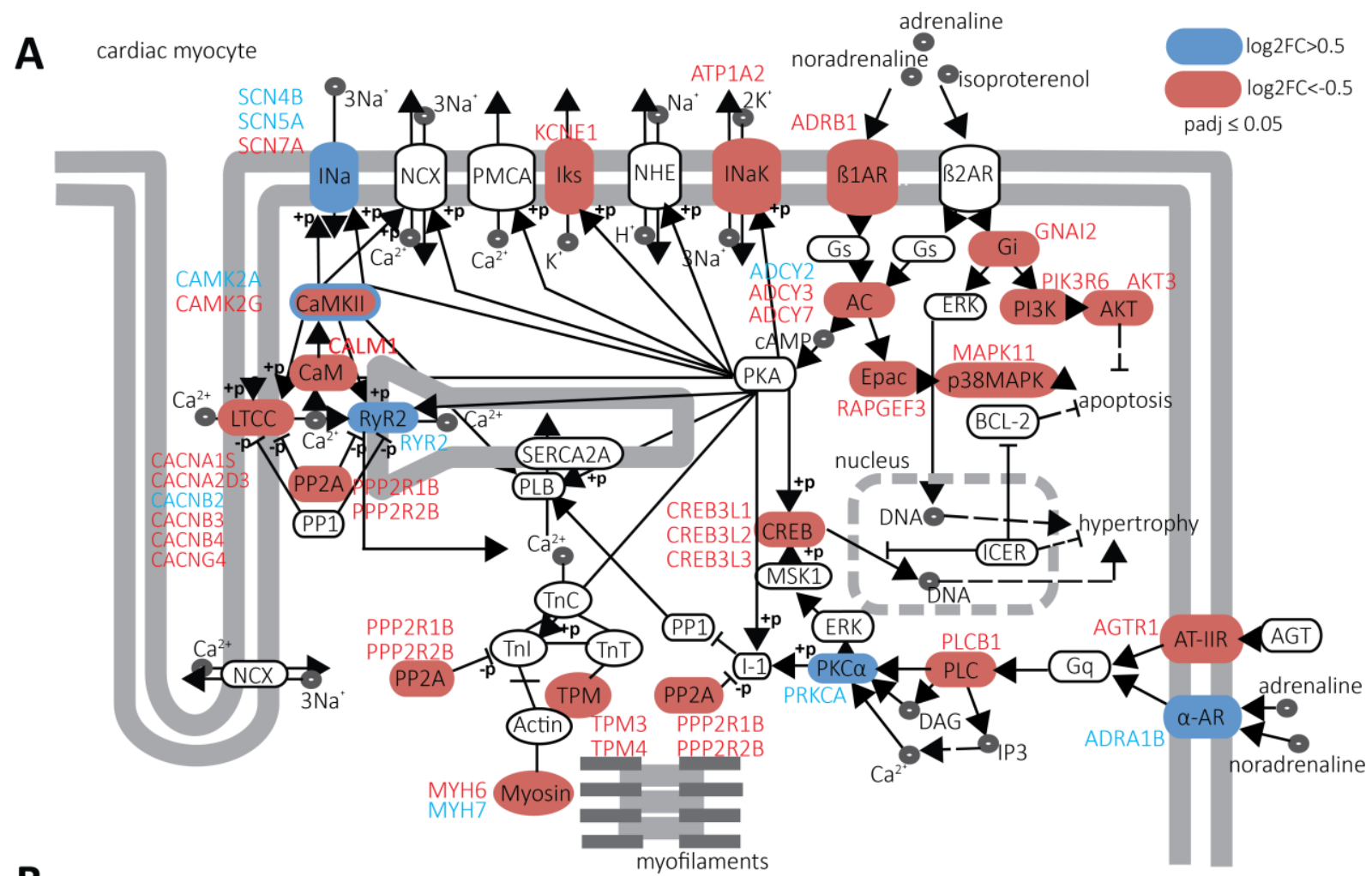

B

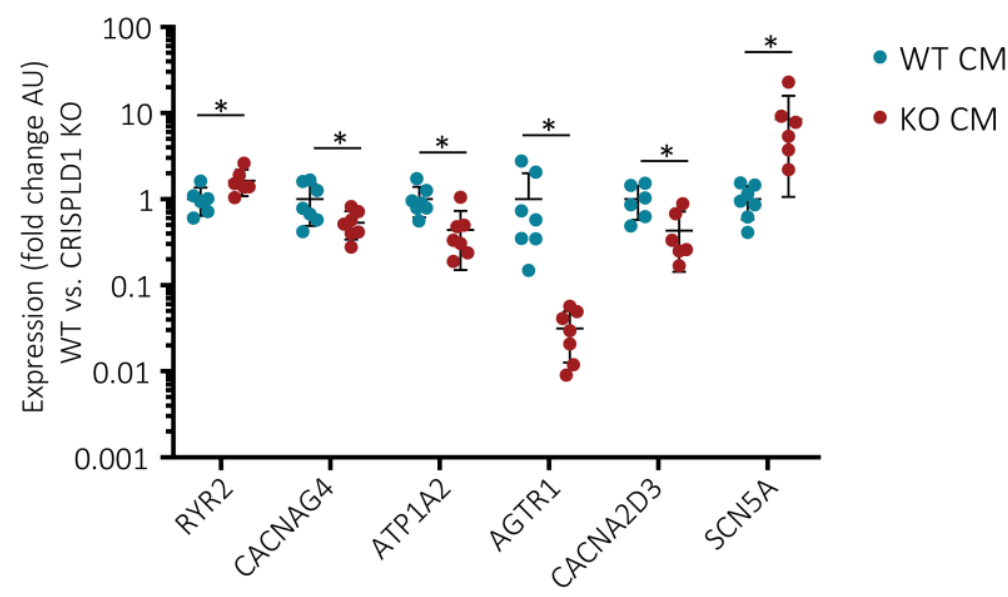

Figure 3-7 DEGs of WT vs. CRISPLD1 KO iPSC-CMs in adrenergic signaling pathway map from KEGG (hsa04261). (A) Most genes in the adrenergic signaling pathway are downregulated (red), and a few upregulated (blue). Genes that are regulated, are written next to the encoding molecule. ADCY2/3/7 = adenylate cyclase 2/3/7, ADRA1B = adrenoceptor alpha 1B, ADRB1 = adrenoreceptor beta 1, AGTR1 = angiotensin II receptor type I, AKT3 = AKT serine/threonine kinase $3, \mathrm{ATP} 1 \mathrm{~A} 2$ = catalytic subunit alpha 2 of the $\mathrm{Na}^{+} / \mathrm{K}^{+}$-ATPase, CACNA1S = calcium voltagegated channel subunit alpha1 S, CACNA2D3 = calcium voltage-gated channel auxiliary subunit alpha2delta 3, CACNB2/3/4 = calcium voltage-gated channel auxiliary subunit beta $2 / 3 / 4$, CACNG4 = calcium voltage-gated channel auxiliary subunit gamma 4, CALM1 = calmodulin 1, CAMK2A/G = calcium/calmodulin dependent protein kinase II alpha/gamma, CREB3L1/2/3 = CAMP responsive element binding protein 3 like 1/2/3, GNAI2 = G protein subunit alpha $\mathrm{i} 2, \mathrm{KCNE} 1$ = potassium voltage-gated channel subfamily $\mathrm{E}$ regulatory subunit $1, \mathrm{MAPK} 11=$ mitogenactivated protein kinase 11, MYH6/7 = myosin heavy chain 6/7, PIK3R6 = phosphoinositide-3-kinase regulatory subunit 6, PLCB1 = phospholipase $\mathrm{C}$ beta 1, PPP2R1B/PPP2R2B = protein phosphatase 2, PRKCA = protein kinase C alpha, RAPGEF3 = Rap guanine nucleotide exchange factor 3, RYR2 = cardiac ryanodine receptor, SCN5A/SCN7A = sodium voltage-gated channel alpha subunit $5 / 7$, SCN4B = sodium voltage-gated channel beta subunit 4 , TPM3/4 = tropomyosin 3/4. (B) Gene expression of representative DEGs relative to GAPDH by RT-qPCR showed significant differences comparing WT and KO CMs. $n=6-7$, where $n$ is number of independent differentiations. Statistics were performed with an unpaired two-tailed t-test. Error bars indicate SD, $*=p<0.05$. Illustration was created by S. Khadjeh and modified by V. Hindmarsh (Khadjeh et al., 2020). 
To validate the RNA-seq data, I carried out proteomics and compared WT iPSC-CMs to CRISPLD1 KO iPSC-CMs at d60. Samples from three independent differentiations were used for analysis. Overall, proteome analysis identified 3201 proteins by mass spectrometry, but only 37 of these proteins were significantly regulated in the WT and CRISPLD1 iPSC-CMs (Data not shown). However, CRISPLD1 was not detected among the identified proteins in the iPSCCMs. When comparing the 3201 identified proteins to the DEGs from RNA-seq, an overlap of 311 shared genes was found (Figure 3-8A). ClueGO analysis of these common genes revealed enrichment of adrenergic signaling, extracellular matrix organization, pathways involved in cardiomyopathies or inflammation and calcium regulation (Figure 3-8B).

A

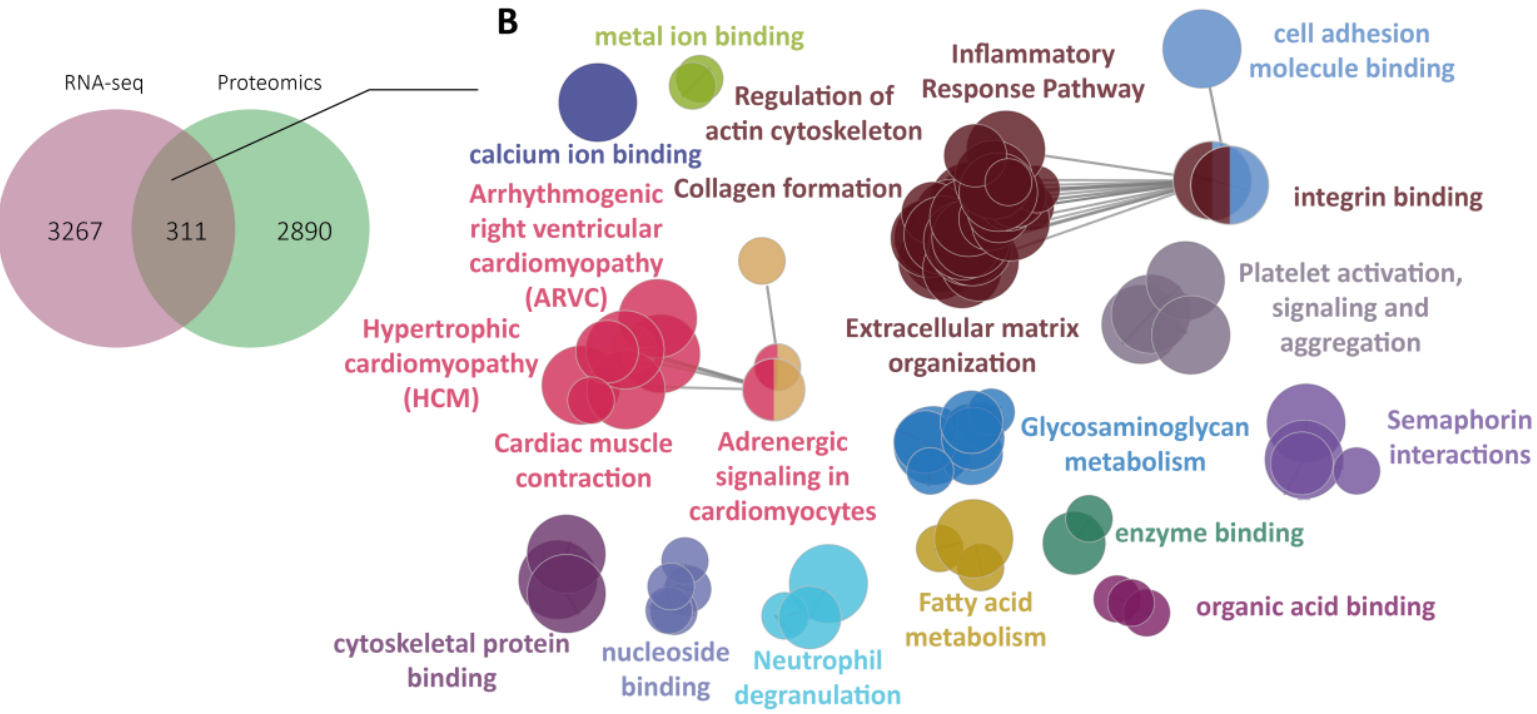

Figure 3-8 Comparison of transcriptomics and proteomics of CRISPLD1 KO iPSC-CMs vs. WT iPSC-CMs. (A) Overlap of DEGs of WT and CRISPLD1 KO iPSC-CMs found in RNA-seq (3267 exclusive DEGs; purple circle) and proteomics (2890 exclusive DEGs; green circle) identified 311 shared genes. (B) ClueGO pathway analysis of the 311 common genes from (A) revealed enrichment of adrenergic signaling in CMs, extracellular matrix organization and pathways of hypertrophic cardiomyopathy. Nodes have been rearranged for better readability. The node size represents the significance of GO term enrichment.

Overall, RNA-seq data and proteomics indicated regulation of apoptotic pathways, inflammatory pathways, cardiomyopathy pathways and calcium regulatory pathways after CRISPLD1 loss-of-function. The identified pathways relate to the observed results found in calcium cycling, indicating an inhibitory role of CRISPLD1 in human cardiac calcium cycling. Detailed analysis of regulated genes in the adrenergic signaling pathway after CRISPLD1 KO support this functional role. 


\subsection{The role of Crispld1 in the mouse heart}

\subsubsection{Generation and validation of Crispld1 KO mice}

Analysis of CRISPLD1 loss-of-function in iPSC-CMs allowed first insights into its role in cardiac cells. For in vivo analysis of its function in mouse, a conditional Crispld1 allele was used. Therefore, a knockout-first conditional mouse was ordered at KOMP Repository (see 2.1.5) which contained two loxP sites flanking exon 5 of Crispld1, a lacZ trapping cassette and a floxed promoter-driven neo cassette (Figure 3-9A). To convert this knockout-first allele into a conditional allele, mice were crossed with a CAG-flpO mice line (Figure 3-9B). FlpO-positive mice that were heterozygous for the floxed allele (Crispld $\left.1^{\mathrm{fl} /+} ; \mathrm{FlpO}^{+}\right)$were then crossed with C57BL/6 WT mice $\left(^{+/+}\right)$to remove the Flp recombinase. The resulting mice, heterozygous for Crispld1 and FlpO negative (Crispld1 ${ }^{\mathrm{fl} /+}$; $\mathrm{FlpO}^{-}$) were then inbred to obtain a homozygous Crispld1 floxed conditional mouse line (Crispld $1^{\mathrm{fl} / \mathrm{fl})}$. This mouse line was further used to obtain a global or a heart-specific Crispld1-deficient mouse line by Cre recombinase (Figure 3-9C), that lacks 39 aa affecting the CAP domain and both V5/Tpx-1 related conserved sites (Figure 3-9D).

A

A

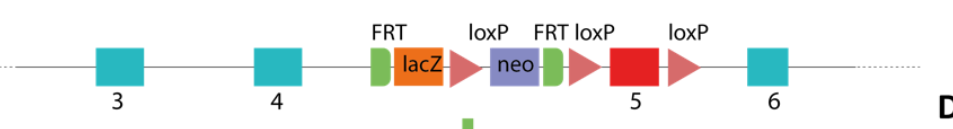

B

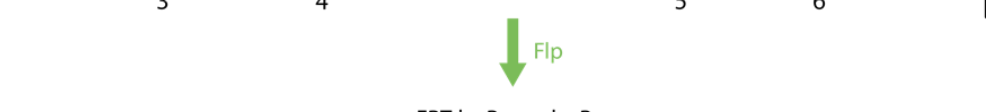

CRISPLD1 structure

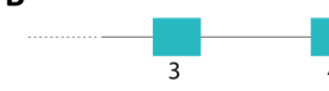

C

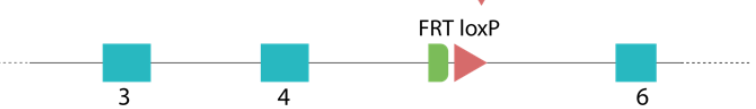

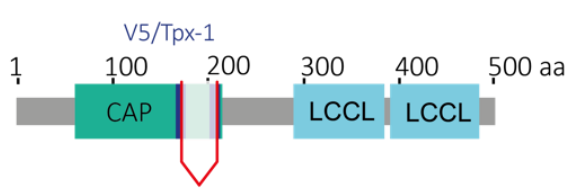

39 aa deletion

Figure 3-9 Generation of the conditional Crispld1 mouse line. (A) Schematic of the knockout-first conditional mouse (KOMP Repository; www.komp.org) that was used to generate a (B) conditional Crispld1 mouse line by Flp recombinase. (C) Cre recombinase deletes Crispld1 exon 5 to generate a knockout mouse. (D) Schematic of expected Crispld1 protein structure after excision of exon 5. Deletion of 39 aa affects the CAP domain and both V5/Tpx-1 related conserved sites.

Once the conditional Crispld1 mouse line was available, where exon 5 is flanked by two loxP sites (Figure 3-10A), matings with either a cardiomyocyte specific Myh6-Cre line or an ubiquitous CMV-Cre line were set up. Therefore, homozygous Crispld1 floxed mice (Crispld1 ${ }^{f / f l}$ ) were crossed to either Myh6-Cre positive mice (Myh6-Cre ${ }^{+}$) or CMV-Cre positive mice $\left(\mathrm{CMV}-\mathrm{Cre}^{+}\right)$to obtain homozygous heart-specific or ubiquitous Crispld1 knockout mice in the F2 generation (Figure 3-10B). The resulting Crispld1 ${ }^{\mathrm{fl} /+} ; \mathrm{Myh}_{-}-\mathrm{Cre}^{+}$or Crispld1 ${ }^{\mathrm{fl} /+} ; \mathrm{CMV}^{-\mathrm{Cre}^{+}}$ 
mice, respectively, were then backcrossed to Crispld $1^{\mathrm{fl} / \mathrm{fl}}$ to obtain homozygous Crispld1 knockout mice that were used for further breeding and experiments. DNA from ear or tail biopsies was used for genotyping (Figure 3-10C-D). Crispld1-flx primers span exon 4 and 5 including the first loxP site. The PCR product was expected to have a size of $514 \mathrm{bp}$ for the floxed allele, $369 \mathrm{bp}$ for the WT allele and no product at all if exon 5 is excised completely. CRISPLD1 KO PCR primers served as additional control in the ubiquitous Crispld1 knockout mouse line and result in a PCR product of $1564 \mathrm{bp}$ for the floxed allele, $1403 \mathrm{bp}$ for the WT allele and $780 \mathrm{bp}$ for the knockout allele. Myh6-Cre or CMV-Cre PCR was important to identify presence of the Cre-recombinase and resulted in a PCR product of $\sim 300 \mathrm{bp}$ if Cre recombinase was present. An internal control for the Myh6-Cre PCR resulted in an additional band of 200 bp and confirmed successful PCR analysis. 


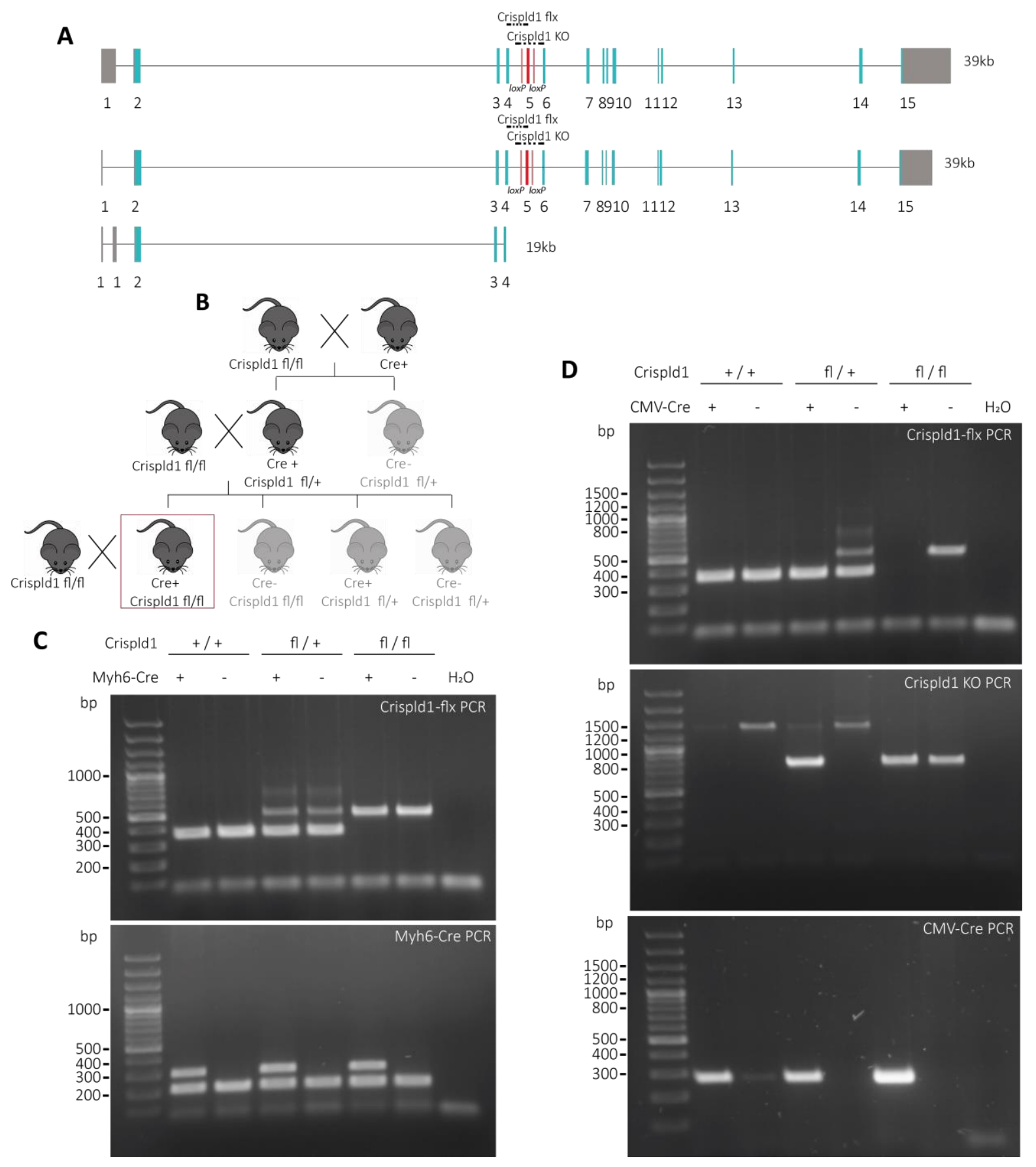

Figure 3-10 Generation of Crispld1 KO mice. (A) Scheme of the three isoforms of the floxed Crispld1 mouse alleles. Exons are represented by either blue or grey boxes for coding or non-coding regions, respectively. The red box represents the target region which is excised after Cre-mediated recombination, the light red boxes indicate the loxP sites. The intron/exon ratio is maintained by the box sizes. The dotted lines indicate the region amplified by the specified primer. (B) Crispld $\mathrm{f}^{\mathrm{fl} / \mathrm{fl}}$ were mated to hemizygous $\mathrm{Cre}^{+}$mice resulting in either Crispld $1^{\mathrm{fl} /++}$; $\mathrm{Cre}^{+}$or Crispld1 $1^{\mathrm{fl} /+}$; Cre . The Crispld $1^{\mathrm{fl} /+} ; \mathrm{Cre}^{+}$mice were crossed to Crispld $1^{\mathrm{fl} / \mathrm{fl}}$ to obtain homozygous KO mice Crispld ${ }^{1 / f f f}$; Cre ${ }^{+}$mice (red box). (C) Representative genotyping PCR of tail biopsies of heart-specific Crispld1 knockout mice. Crispld1-flx primers bind to exon 4 and exon 5, flanking one loxP site, and result in a PCR product of $514 \mathrm{bp}$ for the floxed allele, or $369 \mathrm{bp}$ for the wildtype (WT) allele. For the Myh6-Cre PCR, one primer pair was used as an internal control resulting in a product of $200 \mathrm{bp}$ and the Cre-primer resulting in a product of $\sim 300$ bp. $\mathrm{H}_{2} \mathrm{O}$ served as negative control. (D) Representative genotyping PCR of tail biopsies of ubiquitous Crispld1 knockout mice. Crispld1-flx primers bind to exon 4 and exon 5, flanking one loxP site and result in a PCR product of $514 \mathrm{bp}$ for the floxed allele, $369 \mathrm{bp}$ for the WT allele, or no product if exon 5 is excised completely. Crispld1 KO PCR primers span the region between exon 4 and 6 and result in a PCR product of 1564 bp for the 
floxed allele, $1403 \mathrm{bp}$ for the WT allele and $780 \mathrm{bp}$ after successful recombination. For the CMV-Cre PCR the expected band size is $\sim 300 \mathrm{bp} . \mathrm{H}_{2} \mathrm{O}$ served as negative control. bp = basepairs.

In order to further validate the heart-specific Crispld1 knockout, PCR analyses of heart biopsies and isolated cardiomyocytes were performed (Figure 3-11). Successful Cre-mediated deletion of exon 5 was validated by a smaller band size of about $780 \mathrm{bp}$ for heart-specific KO mice (Myh6xCRISPLD1) heart samples on the PCR gel. In addition, a band size of 1564 bp appeared when CRISPLD1 KO PCR primers span exon 4 and 6 (Figure 3-11A). As PCR was performed on whole heart samples and the Myh6-Cre driver is cardiomyocyte specific, this background DNA band of $1564 \mathrm{bp}$ is due to other cell types than $\mathrm{CMs}$ and therefore still validated a successful recombination. Myh6-Cre and CRISPLD1-floxed (CRISPLD1-flx) mice showed the expected band size of $1403 \mathrm{bp}$ and $1564 \mathrm{bp}$, respectively. Although the Cre-mediated deletion was successful on DNA level, on transcriptome level no significant reduction of Crispld1 mRNA was obtained, neither in the target region, nor in regions outside exon 5 (Figure 3-11C-E). To exclude background mRNA from other cell types than CMs, mRNA levels of isolated cardiomyocytes of heart-specific Crispld1 knockout mice were additionally analyzed. However, also the mRNA level analysis showed no significant reduction of Crispld1 (Figure 3-11B). Sanger sequencing revealed homozygous presence of exon 5 in heart samples suggesting that recombination was not successful although it was validated via PCR before (Figure 3-11F). Due to unavailability of a Crispld1-specific antibody, protein levels could not be investigated by western blot. Furthermore, it was not possible to detect Crispld1 protein in WT mouse samples via proteomics and therefore this analysis for the heart-specific knockout mouse was excluded. Hence, it was not possible to successfully validate the heart-specific Crispld1 knockout. 
A

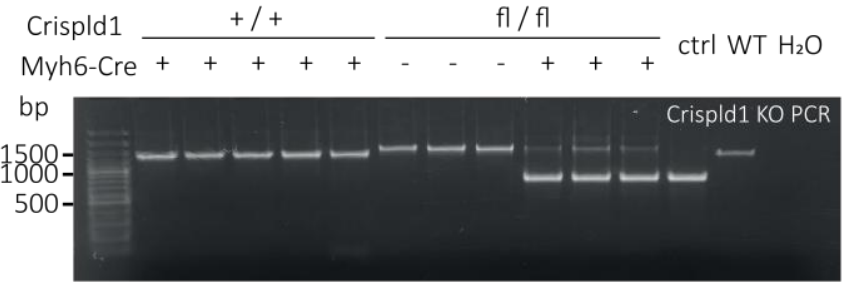

C

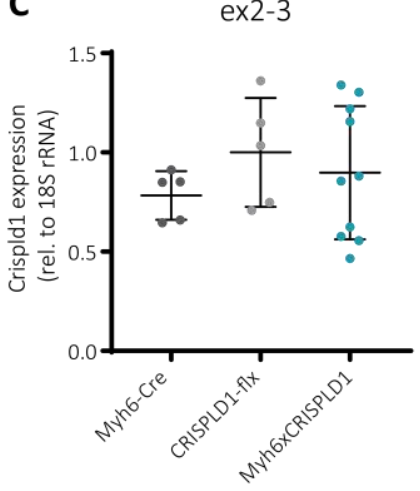

D

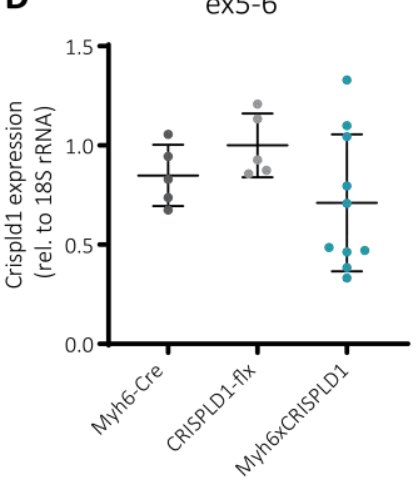

isolated CMs

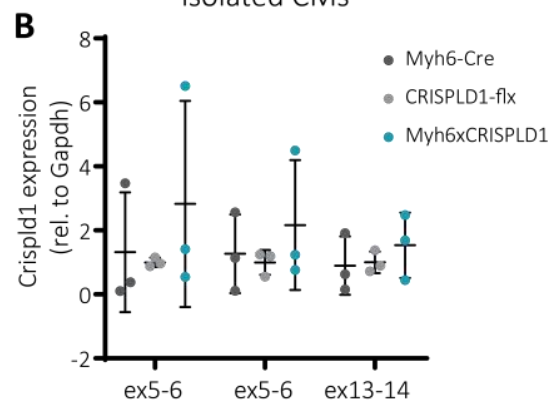

E

ex13-14

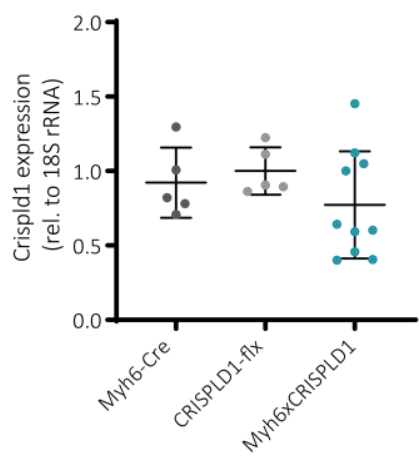

$\mathbf{F}$

heart-specific CRISPLD1 KO- cDNA from heart biopsy

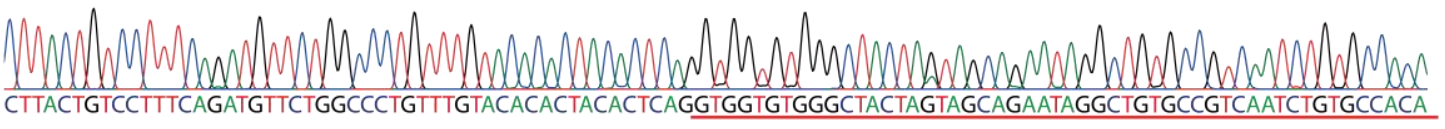
exon 5

Figure 3-11 Validation of heart-specific Crispld1 KO mice. (A) Representative genotyping PCR of heart tissue DNA. Crispld1 KO primers span exon 4 and exon 6 and result in a PCR product of $1564 \mathrm{bp}$ for the floxed allele, $1403 \mathrm{bp}$ for the WT allele and $780 \mathrm{bp}$ after successful recombination. bp = base pairs, ctrl = control sample with successful deletion of exon 5, WT = wild type sample, $\mathrm{H}_{2} \mathrm{O}$ served as negative control. (B) RT-qPCR analysis of Crispld1 expression in isolated mouse CMs using different primer pairs showed no significant reduction of Crispld1 comparing the control lines to the heart-specific KO mouse line. RT-qPCR analysis of Crispld1 expression in heart tissue with primer pairs spanning (C) exon 2-3, (D) the target region exon 5-6 and (E) exon 13-14 showed no significant reduction of Crispld1 mRNA. Crispld1-flx $=1$. (F) Representative Sanger sequencing results of heart sample cDNA from heart-specific Crispld1 KO mice revealed presence of exon 5. Statistics were performed with an unpaired two-tailed t-test. Error bars indicate SD.

The ubiquitous Crispld1 KO was already detected on DNA level in tail biopsies (Figure 3-10D) due to the global deletion of exon 5. Additionally, this knockout was validated on mRNA level of heart samples from ubiquitous Crispld1 KO mice (CMVxCRISPLD1) via RT-qPCR (Figure 3-12A-C). The data showed significant downregulation of Crispld1 expression using primer pairs in the target region and outside the target region, suggesting successful recombination and therefore deletion of exon 5. This result was also validated via Sanger sequencing of heart samples, showing complete deletion of exon 5 (Figure 3-12D). 
A

ex2-3

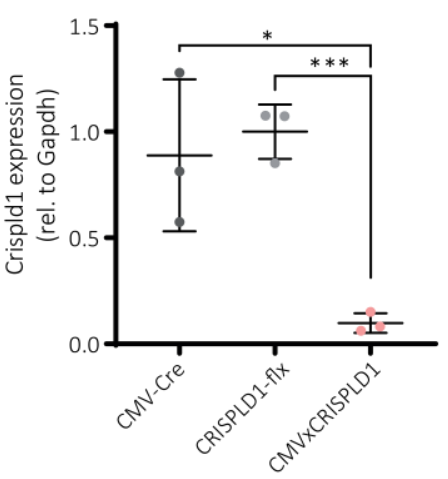

B

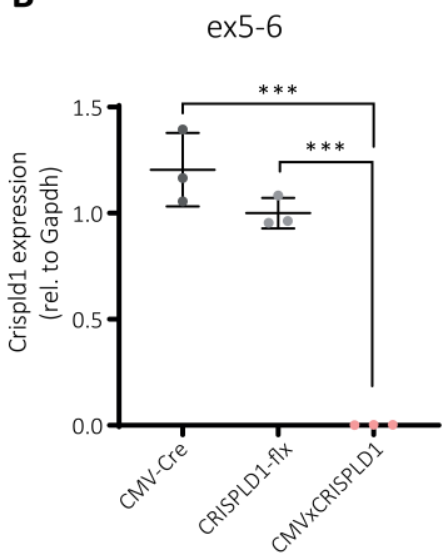

C

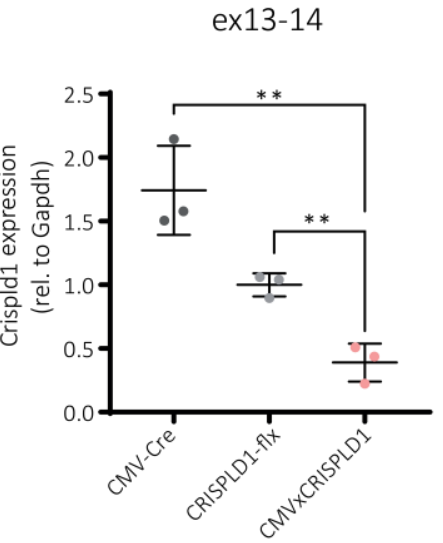

D

ubiquitous CRISPLD1 KO - cDNA from heart biopsy

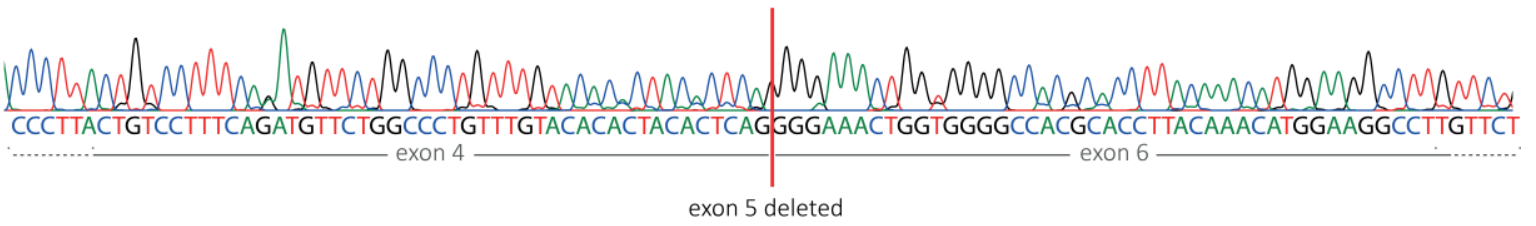

Figure 3-12 Validation of ubiquitous Crispld1 KO mice. RT-qPCR analysis of Crispld1 expression in heart tissue with primer pairs spanning (A) exon 2-3, (B) the target region exon 5-6 and (C) exon 13-14 showed significant reduction of Crispld1 mRNA. Crispld1-flx $=1$. (D) Representative Sanger sequencing results of heart sample from ubiquitous Crispld1 KO mice revealed complete loss of exon 5. Statistics were performed with an unpaired twotailed t-test. Error bars indicate SD, ${ }^{*}=p<0.05 .^{* *}=p<0.01, * * *=p<0.001$.

In summary, validation of Crispld1 knockout by Cre-mediated recombination of two loxP sites leading to a deletion of Crispld1 exon 5 was only successful in the ubiquitous knockout mouse line (CMVxCRISPLD1) but not in the heart-specific knockout mouse line (Myh6xCRISPLD1), although first results on DNA level were promising. However, the validation was only based on DNA and RNA results and could not be performed on protein level due to a commercially unavailable antibody. Therefore, in future studies also the protein level needs to be analyzed to conclusively confirm the ubiquitous Crispld1 knockout.

\subsubsection{The survival of heart-specific Crispld1 KO mice is reduced}

In order to examine the impact of Crispld1 $\mathrm{KO}$ on the mortality, all mouse lines were monitored for up to one year to compare their mortality. Analysis was performed using a Kaplan-Meier curve which revealed a significant higher mortality in the heart-specific Crispld1 KO mouse line in comparison to the remaining ones. Although the Myh6-Cre mouse also showed a reduced survival, there was still a significant difference. Interestingly, the mortality of the ubiquitous Crispld1 $\mathrm{KO}$ line is not affected at all. 


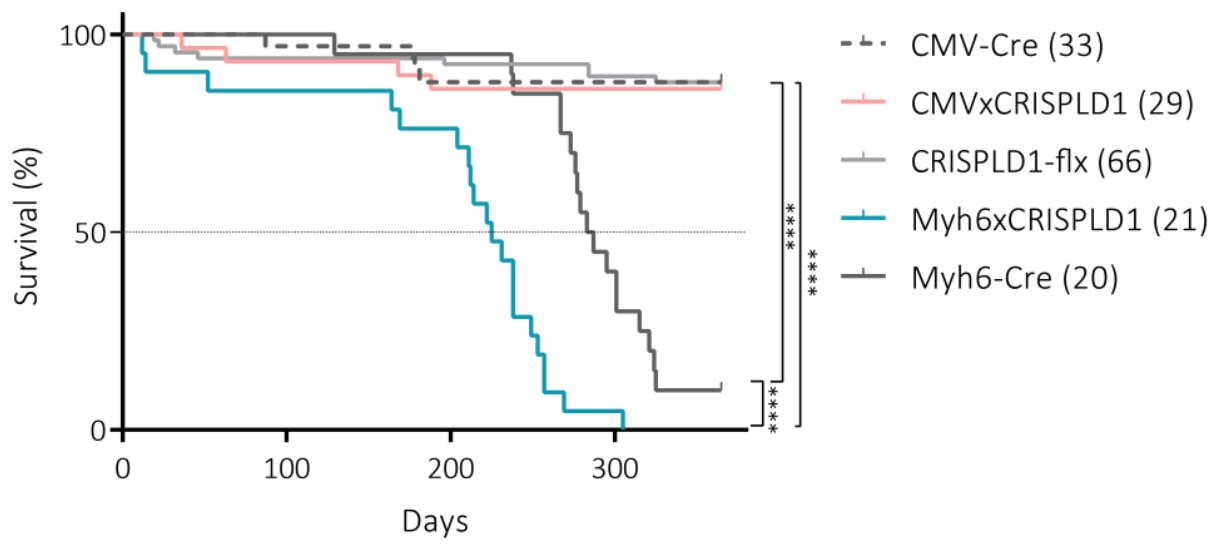

Figure 3-13 Survival curve of Crispld1 KO mouse lines compared to control. The Kaplan-Meier curve represents the percent of living animals for up to one year after birth. Heart-specific Crispld1 KO mice (Myh6xCRISPLD1) show the highest mortality compared to all other lines. $* * * *=p<0.0001$, number in brackets represents number of animals used for analysis. Log-rank Mantel-Cox test used for statistics.

\subsubsection{Heart-specific Crispld1 KO mice show a dilated cardiac phenotype in}

\section{echocardiography}

To investigate the effects of cardiomyocyte-specific as well as ubiquitous Crispld1 depletion and to find an explanation for the high mortality of heart-specific Crispld1 KO mice, echocardiographic characterization was performed. Therefore, the KO mouse lines as well as their controls were compared for their ejection fraction (EF), fractional shortening (FS) and left ventricular diameter (LVEDD) at the age of 24 weeks and 32 weeks. The Myh6xCRISPLD1 mouse line was excluded from analysis at the age of 1 year due to its early death and was therefore only performed in the CMVXCRISPDL1 mouse line with its controls.

Myh6xCRISPLD1 mice showed a significant decreased EF at the age of 24 weeks (mean CMVCre $=46.68$, mean CMVxCRISPLD1 $=49.06$, mean CRISPLD1-flx $=46.96$, mean Myh6xCRISPLD1 $=28.75$, mean Myh6-Cre $=44.99$ ) which deteriorated at the age of 32 weeks (mean CMV-Cre = 45.42, mean CMVxCRISPLD1 = 44.16, mean CRISPLD1-flx = 50.43, mean Myh6xCRISPLD1 = 11.89, mean Myh6-Cre = 38.89). In comparison, CMVxCRISPLD1 mice did not show any alterations compared to the control lines, not even at the age of 1 year ( $\mathrm{CMV}$-Cre $=46.12$, CRISPLD1-flx = 41.70, CMVxCRISPLD1 = 44.90) (Figure 3-14A). Similar results were observed for the FS. The FS was reduced in Myh6xCRISPLD1 mice at 24 weeks (mean CMV-Cre $=23.42$, mean CMVxCRISPLD1 = 24.89, mean CRISPLD1-flx = 23.61, mean Myh6xCRISPLD1 = 13.74, mean Myh6-Cre $=22.29$ ) and worsened at 32 weeks (mean CMV-Cre $=22.85$, mean CMVxCRISPLD1 $=21.88$, mean CRISPLD1-flx $=25.75$, mean Myh6xCRISPLD1 $=5.45$, mean Myh6-Cre = 18.76). CMVxCRISPLD1 mice did not show any changes compared to their controls 
(Figure 3-14B). Lastly, the ventricular diameter was significantly increased in Myh6xCRISPLD1 mice at 24 weeks (mean CMV-Cre $=4.72$, mean CMVxCRISPLD1 $=4.41$, mean CRISPLD1-flx $=$ 4.66, mean Myh6xCRISPLD1 = 5.16, mean Myh6-Cre = 4.44) and 32 weeks (mean CMV-Cre = 4.81, mean CMVxCRISPLD1 = 4.58, mean CRISPLD1-flx = 4.59, mean Myh6xCRISPLD1 = 5.83, mean Myh6-Cre $=4.37$ ). Yet again, CMVxCRISPLD1 mice did not show any significant alterations compared to their controls (Figure 3-14C).

The echocardiographic phenotyping suggests a severe cardiac phenotype in the heart-specific Crispld1 KO mice. These mice developed dilated cardiomyopathy with reduced EF and FS, resulting in an increased risk of heart failure and a high mortality rate. In contrast, ubiquitous Crispld1 KO did not influence the heart function. 
A Ejection fraction
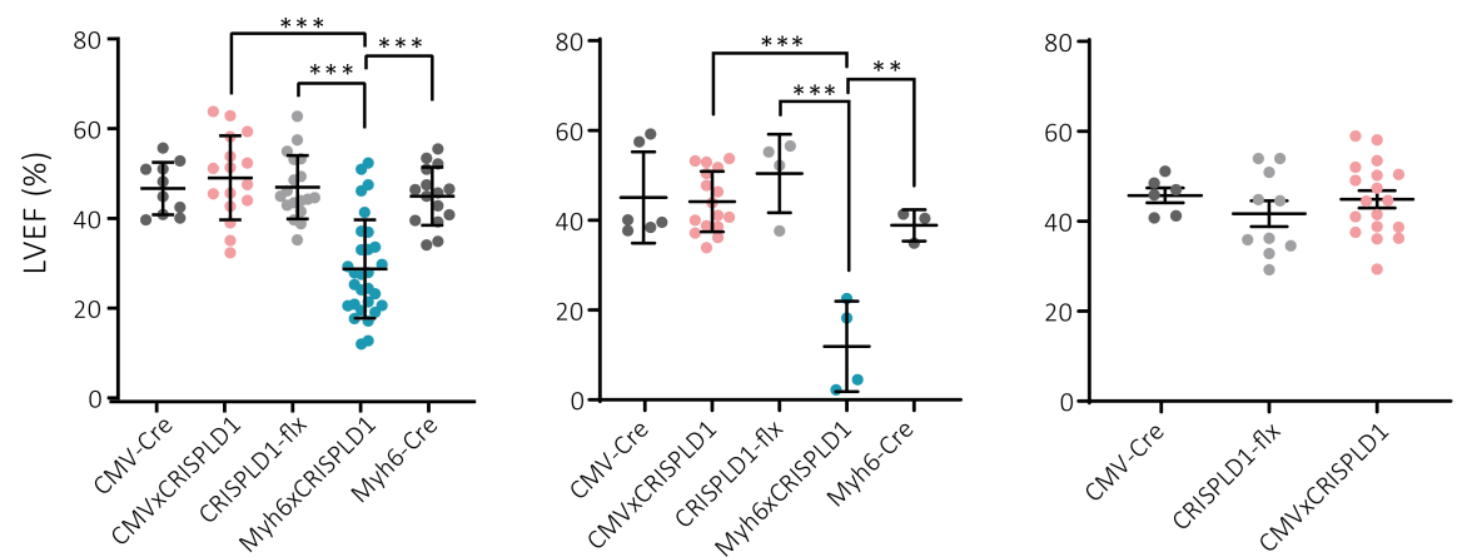

B

\section{Fractional shortening}
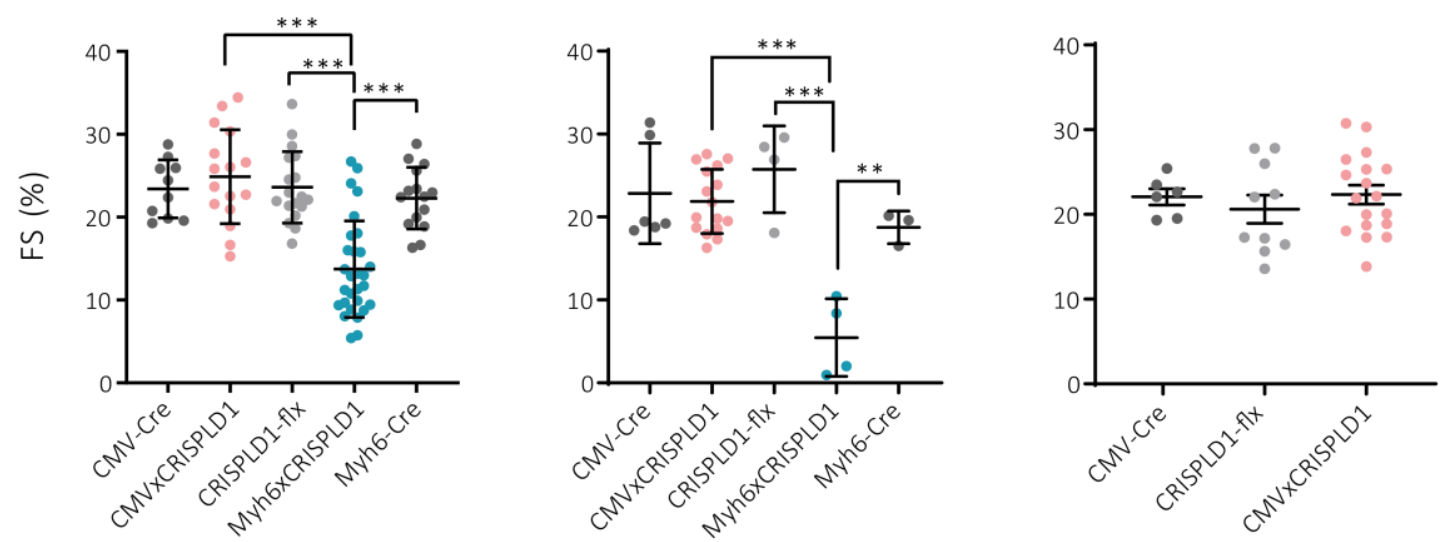

C

Ventricular diameter
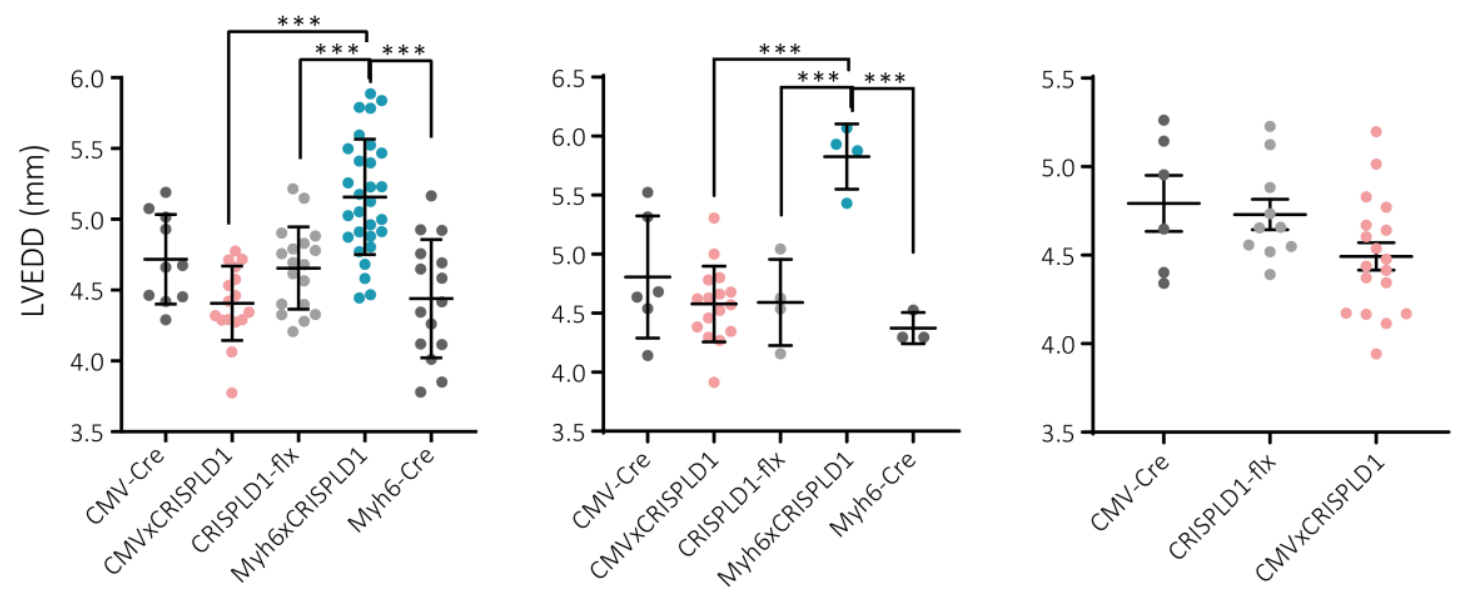

Figure 3-14 Basal echocardiographic phenotyping of Crispld1 KO mouse lines compared to their controls at the age of 24 weeks, 32 weeks and 1 year. (A) The ejection fraction and (B) fractional shortening were significantly reduced in Myh6xCRISPLD1 mice at 24 weeks and 32 weeks. CMVxCRISPLD1 mice did not show any alterations. (C) Myh6xCRISPLD1 mice showed a significantly increased ventricular diameter at 24 weeks and 32 weeks compared to the controls and to CMVxCRISPDL1 which did not show any change. Statistics were performed with a one-way ANOVA together with Tukey post-hoc test. Error bars indicate SD, ${ }^{* *}=p<0.01, * * *=p<0.001$. Each point represents measurement for one mouse. 


\subsubsection{Crispld1 KO does not influence CM size, fibrosis or cardiac gene expression in mice}

In order to further investigate the dilated phenotype of Myh6xCRISPLD1 mice, analysis of CM size, fibrosis and expression of cardiac marker genes was performed. To determine the size of cardiomyocytes, the minimal fiber diameter (MFD) from histological Wheat Germ Agglutinin (WGA) stainings of heart cross sections was measured and compared to the controls (Figure 3-15). However, no difference was observed in MFD (Figure 3-15B) and their distribution in Myh6xCRISPLD1 or CMVxCRISPLD1 mice compared to the controls (Figure 3-15C).

A
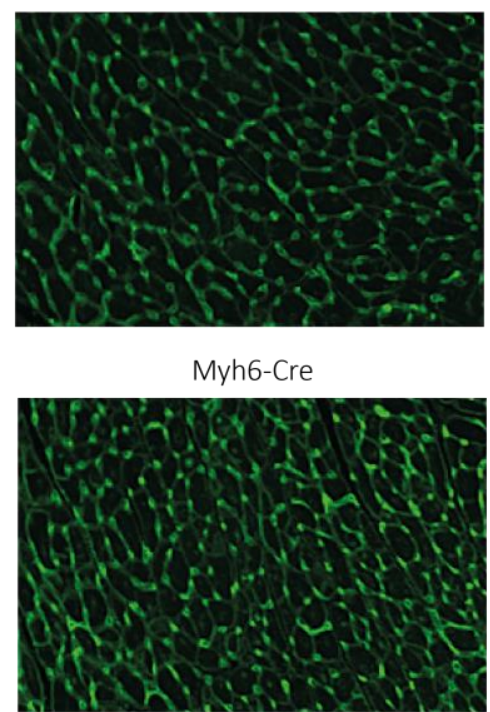

C

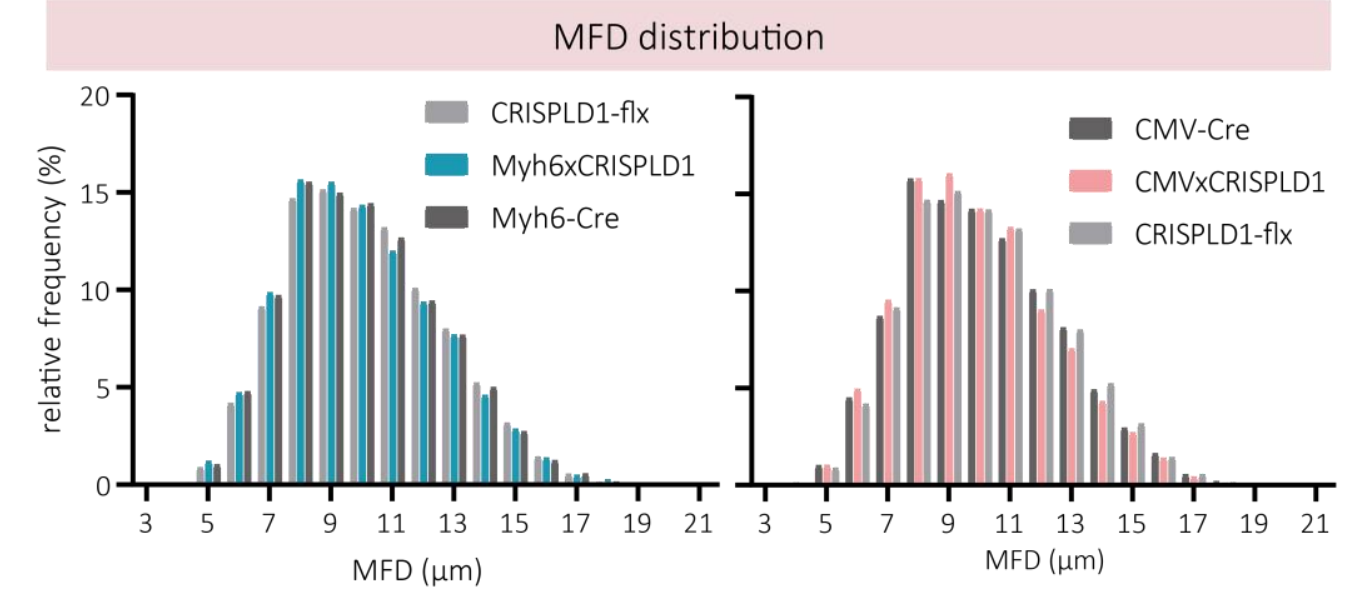

CRISPLD1-flx

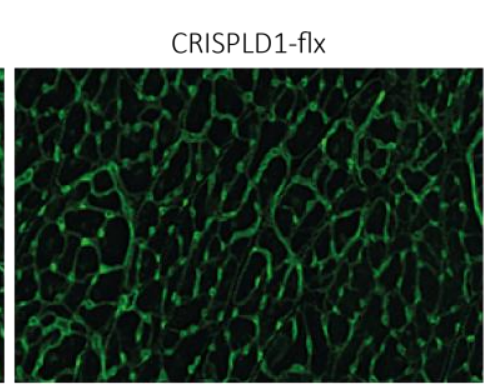

B

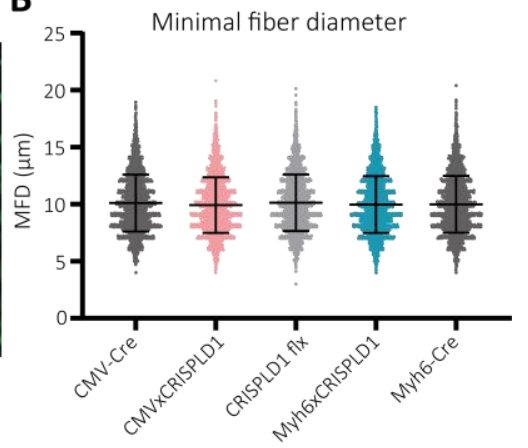

Myh6xCRISPLD1

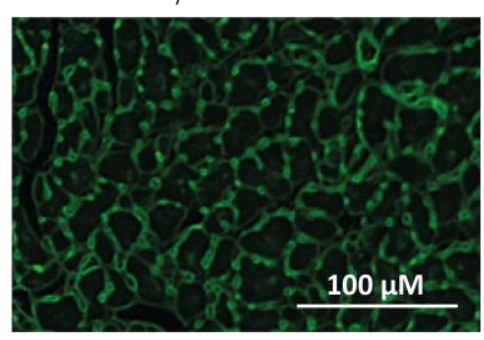

$100 \mu \mathrm{M}$
CMVXCRISPLD1
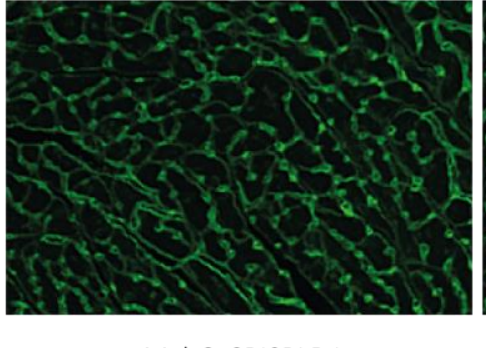

Figure 3-15 Crispld1 KO mice do not show changes in CM size. (A) Representative pictures of Wheat Germ Agglutinin staining. (B) Analysis of minimal fiber diameter (error bars indicate SD) and its (C) distribution does not show any alterations comparing the $\mathrm{KO}$ and control mouse lines.

To visualize and measure fibrotic areas in heart cross sections of 25 weeks old mice, Picro Sirius Red staining was used (Figure 3-16). Semi-automated analysis revealed that there was 
no significant difference comparing the mouse lines (mean CMV-Cre $=9.7$, mean CMVxCRISPLD1 = 4.27, mean CRISPLD1-flx = 4.6, mean Myh6xCRISPLD1 = 11.93, mean Myh6Cre $=6.3)$ (Figure 3-16B). However, big variations across groups influence this result and therefore future studies with an increased number of animals might be necessary to validate this finding.
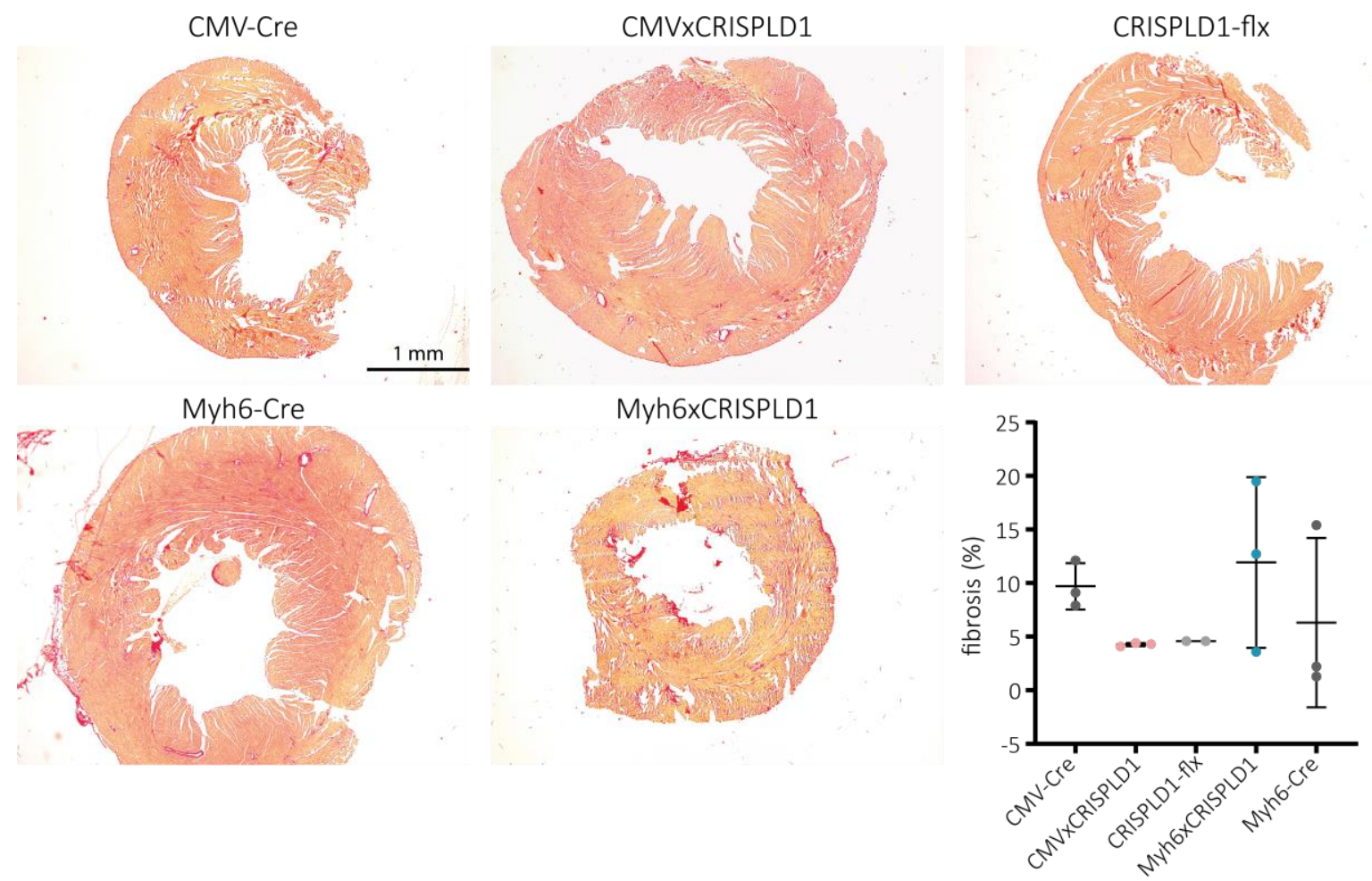

Figure 3-16 Crispld1 KO mice did not show increased fibrosis. (A) Representative images of heart cross sections of 25 weeks old mice stained with Picro Sirius Red. Scale bar $=1 \mathrm{~mm}$. (B) Quantitative analysis of fibrotic areas did not show significant changes comparing the mouse lines. Statistics were performed with an unpaired twotailed t-test. Error bars indicate SD. $\mathrm{n}=3$.

To gain a deeper insight into the molecular events that play a role after Crispld1 KO in the mouse lines and that could lead to the severe cardiac phenotype in Myh6xCRISPDL1 mice, the gene expression of various stress markers was analyzed (Figure 3-17). The hypertrophic gene markers like Nppa and Nppb, both encoding natriuretic peptides, were significantly increased in Myh6xCRISPDL1 mice compared to the CRISPLD1-flx control, but not to the Myh6-Cre control mouse line. Similar results were observed for the CMVxCRISPLD1 mouse line. Nppa and Nppb were significantly increased compared to the CRISPLD1-flx control, but not to the CMV-Cre control. Analysis of Myh6 and Myh7, two myosin heavy chain genes, in Myh6xCRISPLD1 mice showed only significant expressional differences comparing the two control lines but not when compared to the heart-specific Crispld1 KO mouse. Expression 
analysis of Atp2a2, a gene encoding one of the SERCA Ca ${ }^{2+}$-ATPases, did not show any differences. Gene expression of Myh6, Myh7 and Atp2a2 was not changed at all in CMVXCRISPLD1 mice and the control lines.

Although expressional changes were observed for the hypertrophic marker Nppa and Nppb in both Crispld1 knockout lines, these changes might only be due to the background effect of the Cre-recombinase. Changes were only significant when the KO lines were compared to the CRISPLD1-flx control, but not to their Cre-control line. Therefore, it was not yet possible to identify molecular events that happen after Crispdl1 KO in the mouse lines by gene expression analysis of some known cardiac stress markers.
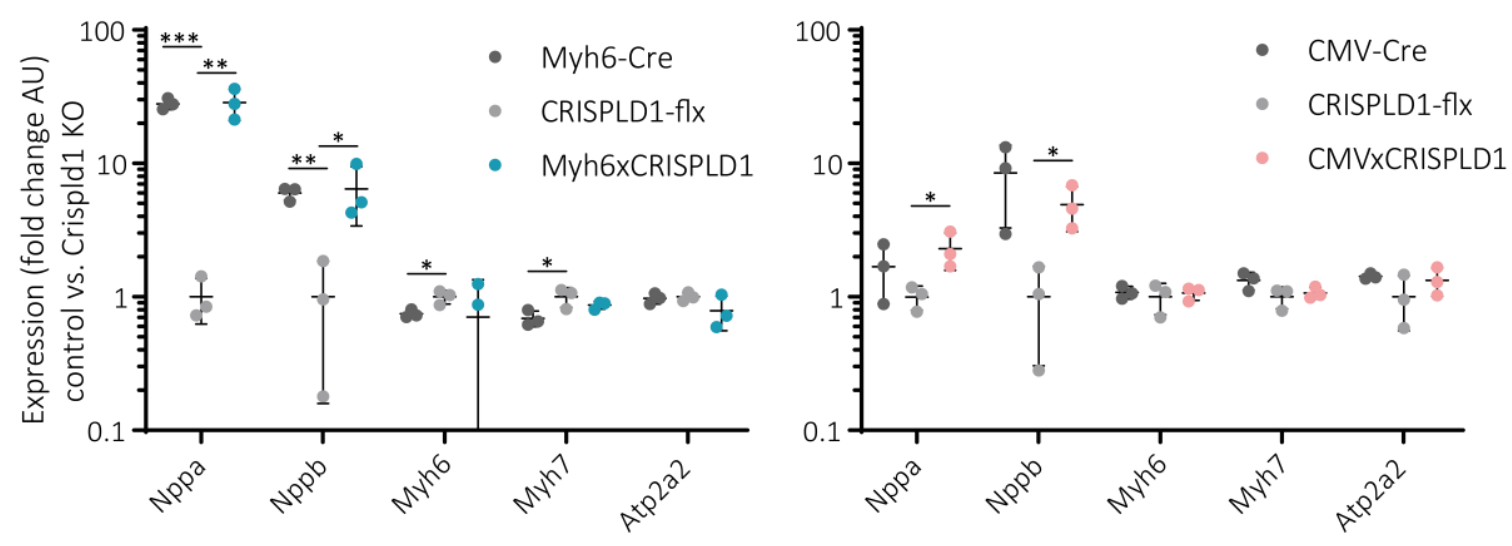

Figure 3-17 Gene expression of cardiac stress markers relative to 185 by RT-qPCR in 25 weeks old Crispld1 KO mice compared to their controls. Analysis showed a significant increase of Nppa compared to Crispld1-flx control but not to Cre-control in Myh6xCRISPLD1 and CMVxCRISPLD1 mice. Similar results were observed for Nppb. $\mathrm{n}=$ 3. Statistics were performed with an unpaired two-tailed t-test. Error bars indicate SD, ${ }^{*}=p<0.05, * *=p<0.01$, $* * *=p<0.001$.

Camkll is an important marker for hypertrophy. It is often upregulated in cardiomyopathies and can therefore be used as a common indicator for a hypertrophic status (Hoch et al., 1999; Wu et al., 2002). Therefore, the protein expression and activity of Camk2a was analyzed by western blot. Protein expression of Camk2a and the phosphorylated Camk2a was not changed in Myh6xCRISPLD1 mice compared to the controls (Figure 3-18B-C). Its activity was measured by comparing phosphorylated Camk2a to unphosphorylated Camk2a and showed a significant increase in Myh6xCRISPLD1 vs. CRISPLD1-flx, but not when compared to the Myh6-Cre control, although the tendency is similar (Figure 3-18D). However, high variances especially within the Myh6-Cre control group influence this outcome and therefore increasing sample size could validate this finding of an increased Camk2a activity in the heart-specific CRISPLD1 KO mouse in the future. 
A
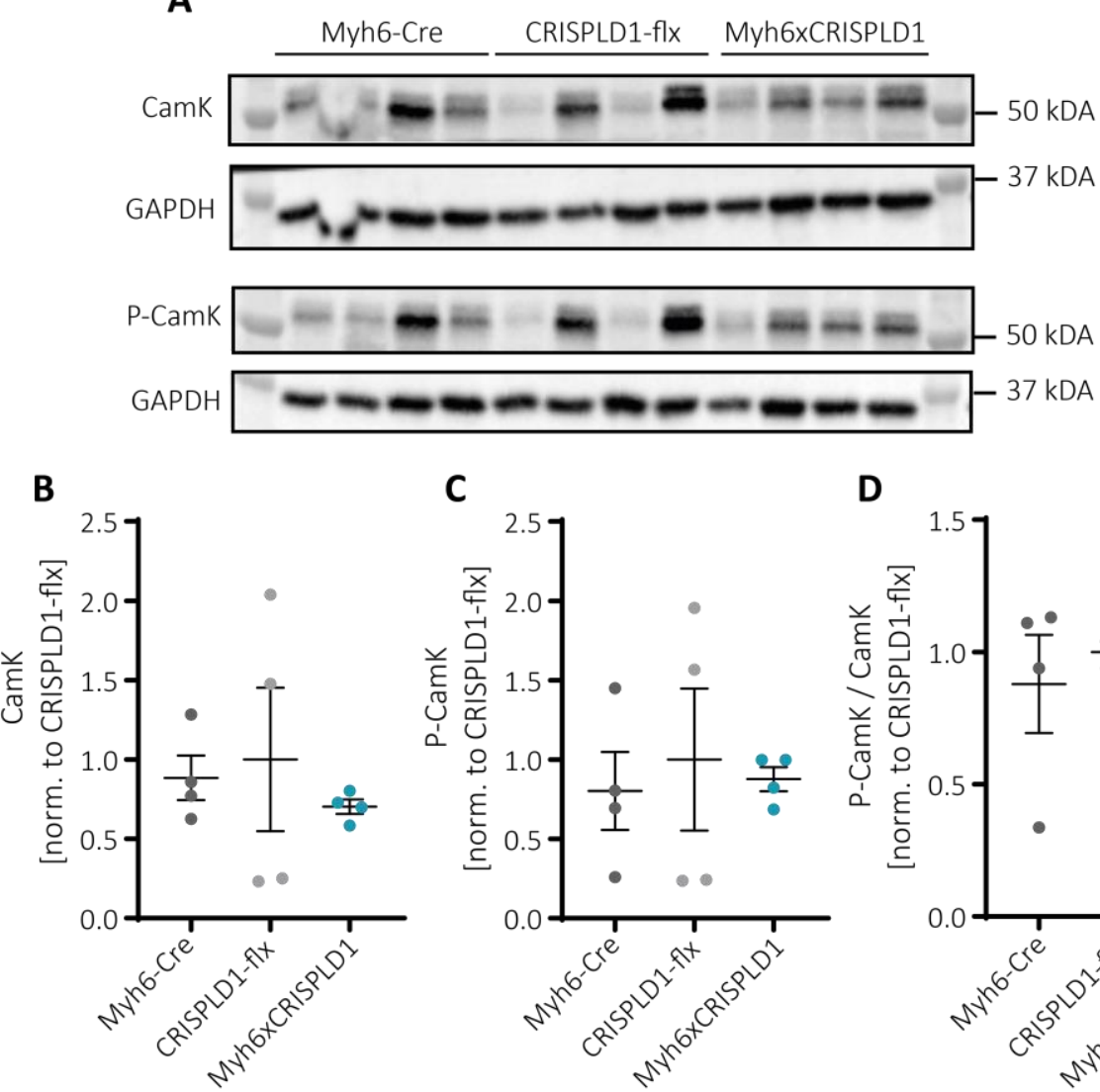

C

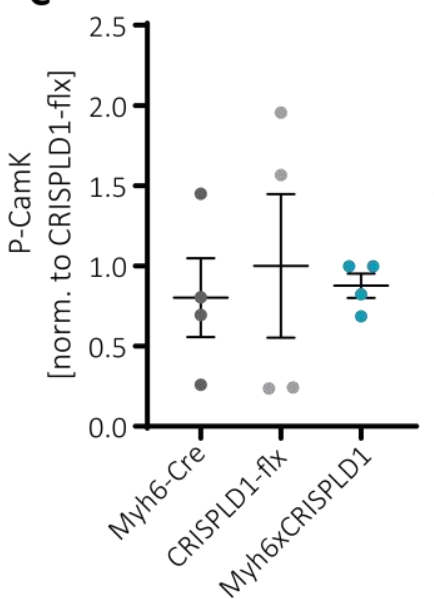

D

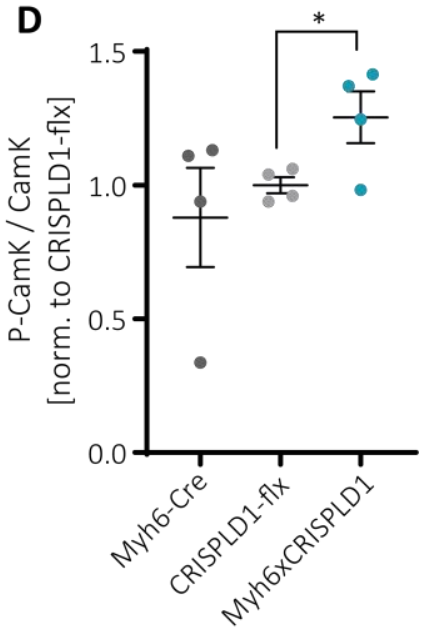

Figure 3-18 Analysis of Camk2a activity in the heart-specific Crispld1 KO mice. (A) Western blot with total protein from left ventricle. (B) Analysis of Camk2a or (C) phosphorylated Camk2a (P-Camk) expression in Myh6xCRISPLD1 vs. control does not show any significant difference. (D) Camk2a is significantly more active in Myh6xCRISPLD1 mice compared to the CRISPLD1-flx control. $n=4$. Statistics were performed with an unpaired two-tailed t-test. Error bars indicate SD, ${ }^{*}=p<0.05$.

Camk2a protein expression in CMVxCRISPLD1 mice was not significantly changed compared to the controls, neither for the inactive, nor for the active form (Figure 3-19B-C). Analysis of the activity by valuating the phosphorylation status of the protein again showed no significant difference in CMVxCRISPDL1 mice (Figure 3-19D).

Taken together, Camk2a seems to be more active in the heart-specific Crispld1 KO mouse, but no changes were observed for the ubiquitous Crispld1 KO mouse. However, repetition of the experiment with an increased sample size is required to validate this finding due to high variances within the groups. 


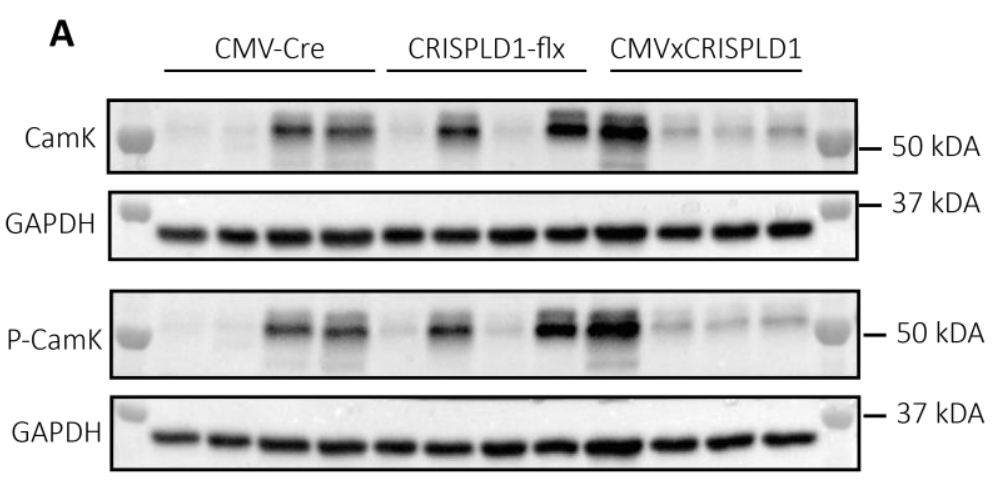

B

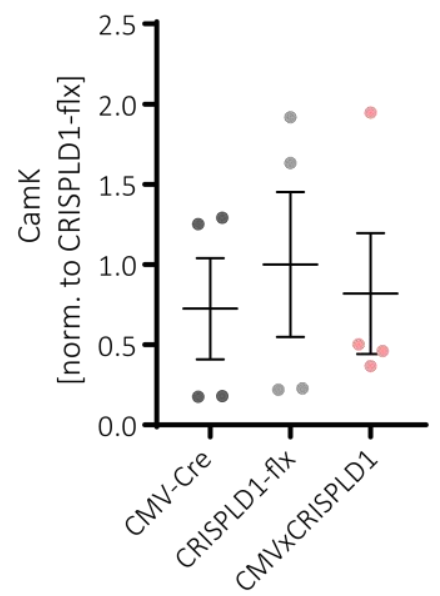

C

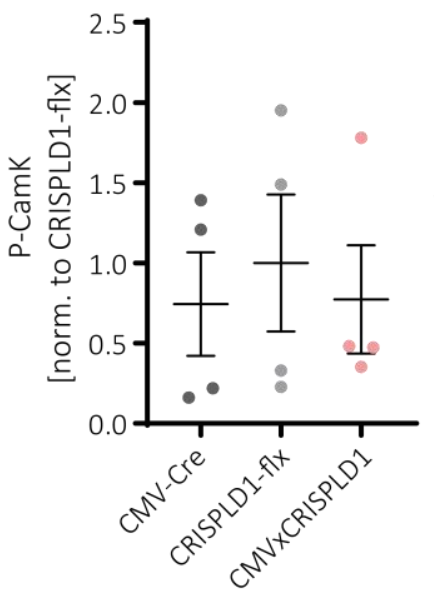

D

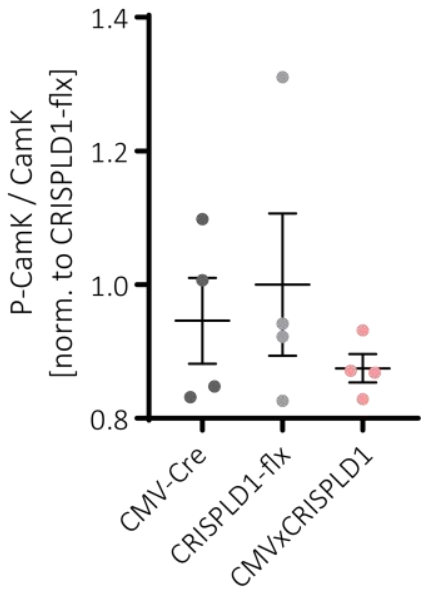

Figure 3-19 Analysis of Camk2a activity in the ubiquitous Crispld1 KO mice. (A) Western blot with total protein from left ventricle. (B) Analysis of Camk2a or (C) phosphorylated Camk2a (P-Camk) expression in CMVxCRISPLD1 vs. control does not show any significant difference. (D) The activity of Camk2a is not changed comparing CMVxCRISPLD1 to the control lines. $n=4$. Statistics were performed with an unpaired two-tailed t-test. Error bars indicate SD.

\subsubsection{Calcium homeostasis is not impaired in Crispld1 KO mouse models}

Results obtained in cell culture showed an inhibitory role of CRISPLD1 KO in iPSC-CMs resulting in an increased CaT amplitude and rise time, but decreased CaT decay 50. To compare these findings to the Crispld1 KO mouse models, the calcium regulation was analyzed in isolated mouse CMs by epifluorescence measurements (Figure 3-20). A significant increase of the amplitude could be seen in CMVxCRISPLD1 and Myh6-Cre compared to the CRISPLD1-flx control at $1 \mathrm{~Hz}$, and in Myh6xCRISPLD1 compared to CRISPLD1-flx control at $4 \mathrm{~Hz}$ (Figure 3-20A). The CaT rise time was not changed at all (Figure 3-20B), whereas the CaT decay 50 showed a significant increase in CMV-Cre and CMVxCRISPLD1 compared to the CRISPLD1-flx control at $2 \mathrm{~Hz}$, and a significant increase in CMVxCRISPLD1 compared to the CRISPLD1-flx control at $4 \mathrm{~Hz}$ (Figure 3-20C). 
However, there was no significant change in the Crispld1 KO mouse lines compared to their Cre-controls, suggesting that the detected differences might be influenced by the Crebackground. Even more, the frequency of stimulation does not influence the outcome. However, as only isolated CMs from 1-2 mice per mouse line were used for analysis, increase of sample size is necessary to validate these findings and hence to exclude that a small sample size masks any effect. 
A

Amplitude
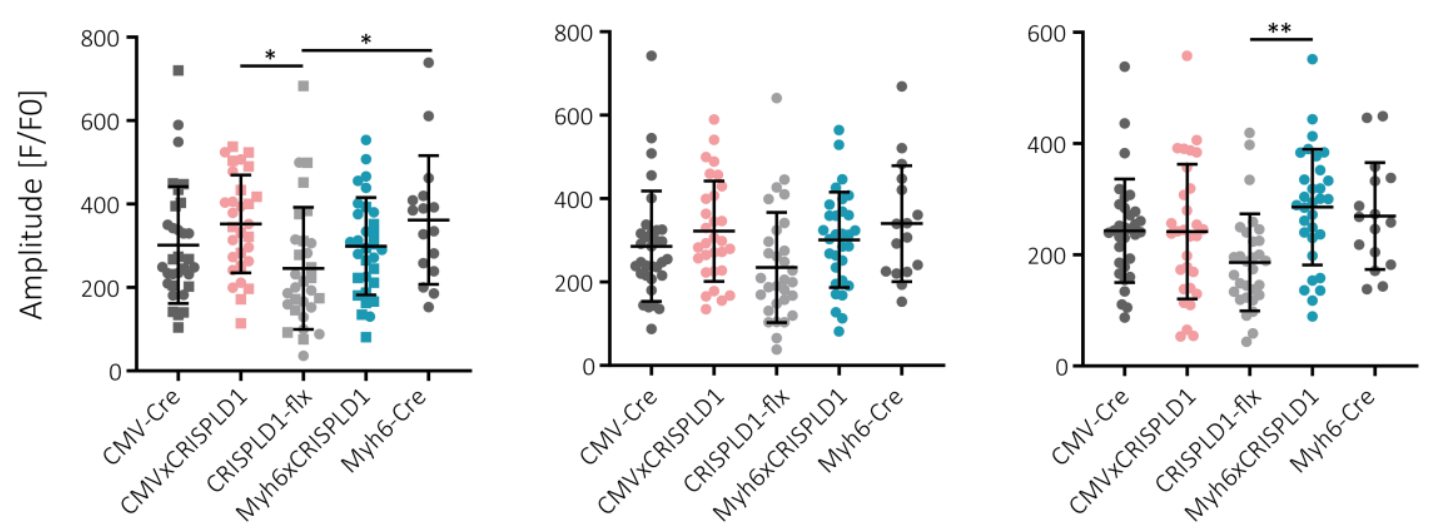

B

CaT rise time
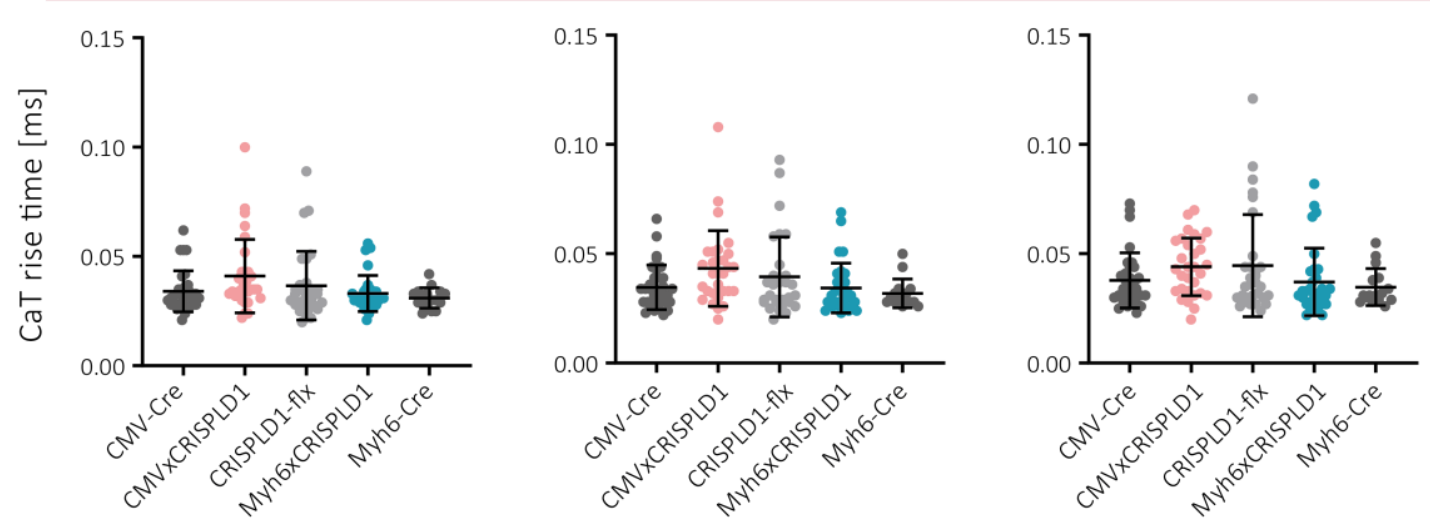

C

Calcium decay 50
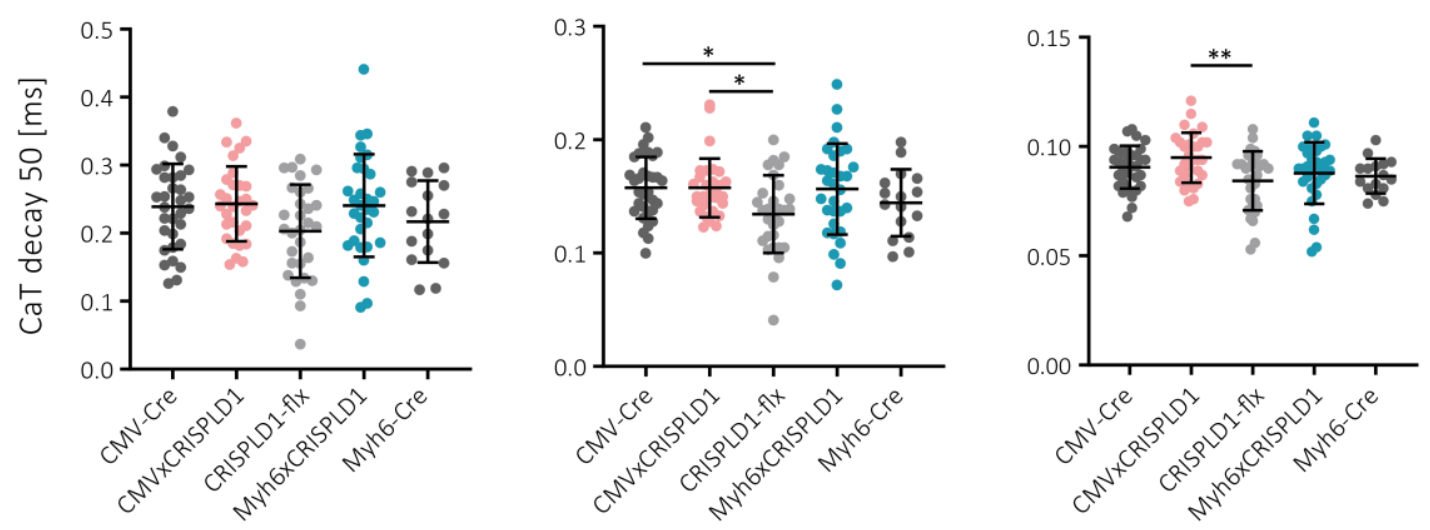

Figure 3-20 Functional analysis of calcium transients of isolated CMs of Crispld1 KO mouse models at $1 \mathrm{~Hz}, 2$ $\mathrm{Hz}$ and $4 \mathrm{~Hz}$. Analysis of $(\mathrm{A})$ the CaT amplitude showed a significant increase in CMVxCRISPLD1 and Myh6-Cre compared to the CRISPLD1-flx control at $1 \mathrm{~Hz}$, and a significant increase in Myh6xCRISPLD1 compared to CRISPLD1-flx control at $4 \mathrm{~Hz}$. (B) The CaT rise time was not changed comparing the mouse lines. (C) CaT decay 50 was significantly increased in CMV-Cre and CMVxCRISPLD1 compared to CRISPLD1-flx control at $2 \mathrm{~Hz}$, and in CMVxCRISPLD1 compared to CRISPLD1-flx at $4 \mathrm{~Hz}$. Error bars indicate SD, ${ }^{*}=p<0.05 ; * *=p<0.01 . n=16-32$, where $\mathrm{n}$ is the number of measured isolated CMs. 


\section{Discussion}

For the last 20 years, cardiac diseases were the main cause of hospitalization and cause of death worldwide. Among these, heart failure, a multifactorial disease with a poor outcome is the major health burden that leads to immense healthcare expenditures which are expected to more than double in the next years (Lippi \& Sanchis-Gomar, 2020; WHO, 2020). Although various therapeutics already exist, the morbidity and mortality are still very high. Therefore, new therapeutic targets and strategies are needed to enhance the clinical outcome.

Analysis of human myocardium biopsy samples from aortic stenosis patients at the hypertrophic and heart failure state compared to healthy donors allowed expression profiling during the progression from healthy hearts to heart failure. Data was compared to analogous myocardium samples from transverse aortic constriction (TAC) mice and identified 25 genes, that showed conserved expression in human and mouse, and regulated expression depending on the disease stage. Among these genes, CRISPLD1 was identified as a new regulator as it showed increasing expression during the transition to heart failure in human and mouse and is functionally unknown in the heart environment.

\subsection{The role of CRISPLD1 in iPSC-CMs}

The specific function of CRISPLD1 is mainly unknown in any type of disease, organ or cell. However, CRISPLD1 has been identified to play a role in different pathways like the folic acid pathway (Chiquet et al., 2011), or in progesterone resistance in endometrial cancer (Li et al., 2019). It is one of the regulators in murine hematopoietic stem cell repopulation and regulated in chondrocyte's response to IL-1 $\alpha$ (Holmfeldt et al., 2016; Wilson et al., 2016). It was further found in prostatic secretions (Principe et al., 2013) and human parotic glands (Gonzalez-Begne et al., 2009). Furthermore, CRISPLD1 showed an altered methylation pattern during male infertility (Sujit et al., 2018). In the heart, it was only recently described as playing a key role in human platelet transcriptome in patients with acute myocardial infarction (Zhang et al., 2021) and that CRISPLD1 polymorphism regulates antiplatelet effects of clopidrogel in Chinese Han coronary heart disease patients (Wang et al., 2018b).

CRISPLD1 belongs to the superfamily of CAP proteins which are often secreted and work in an autocrine or paracrine manner. The structural conservation of this superfamily results in similar functions of the CAP domain of all members (Gibbs et al., 2008). Few of these were 
identified to play a role in cardiovascular diseases like the peptidase inhibitor 16 (PI16) that regulates the CM size negatively and has a role in HF remodeling (Frost \& Engelhardt, 2007). CRISPs, which are a subfamily of the CAPs, have the unique feature of regulating ion channels like voltage-gated calcium channel, voltage-gated potassium channel or the ryanodine receptor. They can mostly be found in the male reproductive tract and in the venom of poisonous reptiles (Gibbs et al., 2008). One example is helothermine, a toxin found in the venom of the lizard Heloderma horridum horridum (Mochca-Morales et al., 1990) that showed a high conservation to CRISPLD1 and also contains the two signatures CRISP1 and CRISP2, that are related to the two V5/Tpx-1 sites of CRISPLD1 (PROSITE patterns PS01009 and PS01010) (Figure 1-6B). Therefore, CRISPLD1 was selected as the candidate gene to study.

\subsubsection{The influence of CRISPLD1-deficient iPSC-CMs on the calcium homeostasis}

First of all, CRISPLD1 was characterized in iPSC-CMs to identify its function in cardiomyocytes. Using the CRISPR/Cas9 system, a CRISPLD1 knockout cell line with a deletion of 34 bp in exon 7 was generated, that resulted in a deletion of $303 \mathrm{bp}$ in the mRNA and therefore deletion of three exons due to exon skipping. However, parts of the CAP domain and V5/Tpx-1 related site were lost and the remaining mRNA significantly downregulated. Due to a commercially unavailable antibody and absence in proteome sequencing, even in WT, it was not possible to validate the knockout or its downregulation on protein level. Nevertheless, because of the downregulation on mRNA level and expected loss of parts of the protein, I assume a functional knockout that could specifically be characterized regarding loss of parts of the CAP domain and V5/Tpx-1 related sites (Figure 3-1).

The differentiation efficiency or morphology of the KO iPSC-CMs were not visibly interfered. CRISPLD1 seems to not have an important influence on the differentiation process of iPSCS into functionally beating CMs, neither on the sarcomeric structure which still showed a high degree of organization. Due to the homology to known calcium regulators, I decided to study the calcium cycling in CRISPLD1 KO iPSC-CMs. CRISPLD1 seems to regulate calcium negatively. After CRISPLD1 KO, the CaT amplitude and rise time showed a significant increase, whereas the CaT decay 50 was significantly decreased. This suggests an increased systolic CaT due to the loss of CRISPLD1 function. I further wanted to validate these results obtained from calcium imaging and test whether CRISPLD1 might influence the effect of the related toxin helothermine on calcium cycling. Helothermine is known to block the cardiac ryanodine 
receptor and therefore regulates calcium (Morrissette et al., 1995). First, I tested the influence of helothermine on CaT in WT iPSC-CMs to identify the best concentration to work with. I observed that only concentrations of $2,5 \mu \mathrm{M}$ and $5 \mu \mathrm{M}$ influence the CaT amplitude and therefore selected $5 \mu \mathrm{M}$ for further experiments. Helothermine treatment resulted in decreased CaT amplitude and significantly increased CaT rise time and CaT decay 50 in WT iPSC-CMs. When helothermine was applied to CRISPLD1 KO iPSC-CMs I observed a rescue of this helothermine effect on WT CaT resulting in normalized CaT amplitude and CaT rise time. Although the CaT decay 50 was not fully normalized to WT level, the effect was significantly less compared to helothermine treated WT iPSC-CMs.

Therefore, I could see a measurable effect of CRISPLD1 loss on CaT characteristics of iPSC-CMs. Partial CRISPLD1 loss together with downregulation of the remaining mRNA resulted in increased CaT which could rescue the CaT phenotype after toxin treatment. These results support the functional role of CRISPLD1 as an inhibitor in human calcium cycling. Its expressional increase during the transition from healthy hearts to hypertrophy and to heart failure in aortic stenosis patients suggests a role as a negative calcium regulator. However, the exact localization or molecular mechanism and targets of CRISPLD1 are still unknown and need to be analyzed in future experiments to validate and support the findings.

\subsubsection{CRISPLD1 influences hypertrophic, apoptotic and calcium regulating pathways}

In order to get a deeper insight into the pathways in which CRISPLD1 could be involved in, RNA-seq was performed. Prohypertrophic and proapoptotic pathways like G-protein signaling, CREB signaling, WNT signaling, TGF-beta signaling, TNF signaling and TP53 signaling were downregulated, suggesting a beneficial effect of CRISPLD1 KO in iPSC-CMs. Even more, ADRB1, which plays an important role in $\mathrm{HF}$ as overstimulation of adrenergic signaling results in $\mathrm{CM}$ disturbances (Braunwald \& Chidsey, 1965; Osadchii, 2007) is downregulated, suggesting a protective mechanism in HF. Downstream targets of this ß-adrenergic receptor like genes encoding AC, Epac, p38MAPK, Gi, PI3K or AKT are also downregulated, further supporting the assumption of a beneficial CRISPLD1 KO. Heart failure treatments often target this signaling pathway by using beta-blockers, that mainly act on beta-adrenergic receptor genes like ADRB1 (Shin \& Johnson, 2007). Polymorphism of this gene was shown to influence the therapy and outcome of heart failure indicating the importance of individual therapy and identification of 
additional genes contributing to this response variability (Pacanowski et al., 2008; Parikh et al., 2018).

After CRISPLD1 KO, Angiotensin II receptor type 1 (AGTR1) is significantly downregulated in the transcriptome. This gene is known to be involved in coronary heart diseases (CHD) (Li et al., 2013; Li et al., 2020). Polymorphism was associated with the development of coronary artery disease (CAD) and AGTR1 methylation, which regulated its expression negatively, was elevated in CHD patients (Li et al., 2020). Interestingly, ADRA1B, which was shown to cause maladaptive response in PO after loss-of-function (O'Connell et al., 2006), was significantly upregulated after CRISPLD1 KO, supporting again the beneficial effect of CRISPLD1 loss-offunction in adrenergic signaling.

As expected, due to the calcium transient changes after CRISPLD1 KO, calcium regulating genes showed alterations in the transcriptome. The genes SCN5A and SCN5B, encoding the cardiac sodium channel, are upregulated, whereas KCNE1, which was shown to prolong the QT interval in HF patients when being upregulated (Watanabe et al., 2007), and ATP1A2 encoding the sodium-potassium ATPase, are downregulated. After CRISPLD1 loss-of-function, the L-type $\mathrm{Ca}^{2+}$-channel (LTCC) was significantly downregulated contrary to the upregulation of RYR2. Calcium influx via the LTCC is essential to initiate excitation-contraction coupling and therefore regulates contraction (Luo \& Anderson, 2013; Eisner et al., 2017). Abnormalities lead to contractile dysfunction (Chen et al., 2002) and hyperactivation of the LTCC was only recently shown to increase cellular hypertrophy in right ventricular myocytes in rats with $\mathrm{HF}$ (Medvedev et al., 2021). However, as some publications reported increased LTCC in hypertrophy and heart failure, others showed unchanged or downregulated LTCC in HF (Ather et al., 2013). Additionally, Goonasekera et al. demonstrated that downregulated LTCC resulted in RYR2-mediated SR Ca ${ }^{2+}$ leak and together with increased neuroendocrine expression preserved contractility as a compensatory mechanism. However, in the long term this leads to hypertrophic remodeling (Goonasekera et al., 2012). To sum up, all of these transcriptional changes, especially changes in the calcium regulating genes, could give an idea about the functional changes that influence the calcium regulation observed by altered CaT in CRISPLD1 KO iPSC-CMs. 
To further validate these findings in RNA-seq analysis and to try to validate CRISPLD1 KO on proteome level independent of western blot analysis, I performed additional proteome sequencing. Interestingly, although 3201 proteins were identified, excluding CRISPLD1, only 311 showed an overlap with the corresponding genes from the RNA-seq data. These overlapping genes were mainly involved in adrenergic signaling in CMs, extracellular matrix organization and pathways of hypertrophic cardiomyopathy, similar to our findings in the transcriptome. However, only 37 of the 3201 proteins were significantly regulated from which only 17 were overlapping to the transcriptome data and were therefore significantly regulated in both analyses. This discrepancy between transcriptome and proteome analysis indicates, that pathway analysis of the transcriptome might not always reveal the actual situation. This is due to various regulatory mechanisms like post-translational modifications, alternative splicing or protein degradation (Sousa Abreu et al., 2009; Zapalska-Sozoniuk et al., 2019). mRNA levels do not always correlate to the protein levels, or rather many protein levels cannot be predicted from mRNA levels because the main step of determining the protein level is the posttranscriptional regulation (Ghaemmaghami et al., 2003; Zapalska-Sozoniuk et al., 2019). Therefore, this step could be responsible, at least partially, for the discrepancies between transcriptome and proteome findings. It should also be taken into account, that proteomics do not identify all proteins that are present in the cell. This means that there is always a lack of information influencing the results.

Transcriptome and proteome analysis could nevertheless be a good way to get an idea about pathway changes and set a starting point. Further validation by functional analysis like western blot of calcium regulators or analysis of posttranscriptional regulations is essential to validate these findings and to close the gap between transcriptomics and proteomics.

\subsubsection{CRISPLD1 has a functional role in the transition to $\mathrm{HF}$}

Overall, I hypothesize that the increased CaT amplitude and the faster $\mathrm{Ca}^{2+}$-efflux after CRISPLD1 KO could result in a beneficial effect in response to CM stress. This hypothesis is further supported by the findings after helothermine treatment, where the calcium blocking toxin effect was rescued by CRISPLD1 KO. Transcriptome data together with pathway analysis further support this hypothesis, although there is still the gap to proteomics. However, the detailed molecular mechanisms, the localization and the downstream targets of CRISPLD1 remain unknown and need to be validated in future studies. Furthermore, the effect of 
affected calcium cycling on the contractile function and action potential needs to be elucidated to validate a beneficial effect. Therefore, future studies regarding measurement of the action potential, intracellular $\mathrm{Ca}^{2+}-\mathrm{load}, \mathrm{SR} \mathrm{Ca}{ }^{2+}-\mathrm{load}$ and $\mathrm{Ca}^{2+}$-currents should further explain and validate the role of CRISPLD1 in iPSC-CMs and its specific function during disease development.

\subsection{The role of Crispld1 in the mouse heart}

\subsubsection{Generation of Crispld1 KO mice could not be validated in the heart-specific KO line,} but showed a downregulated truncated mRNA in the ubiquitous KO mouse line

In order to validate our findings obtained in cell culture experiments and to compare to data from a functional organism, two conditional Crispld1 KO mouse lines were generated. As a knockout-first mouse line was ordered from KOMP repository, I first transformed this line into a conditional mouse line by using the Flp Recombinase followed by inbreeding to obtain the homozygous CRISPLD1-flx mouse line. This mouse line was either bred with a Myh6-Cre line, expressing a constitutively active Cre recombinase specifically in CMs (Agah et al., 1997), or with a CMV-Cre line, expressing Cre ubiquitously (Schwenk et al., 1995). Both lines resulted in a successful deletion of Crispld1 exon 5 on DNA level.

To validate both KO models, I performed RT-qPCR analysis of heart biopsies and compared Crispld1 expression of the KO mouse lines to isogenic controls using primers in- and outside the deleted exon. Crispld1 KO of exon 5 could successfully be validated for the ubiquitous knockout line in DNA and RNA samples and further showed significant decreased expression of the Crispld1 remaining mRNA. Additionally, Sanger sequencing confirmed a complete inframe deletion of exon 5, indicating expression of a truncated Crispld1 mRNA in the ubiquitous Crispld1 KO mouse line. However, it was not possible to validate Crispld1 exon 5 deletion or at least a significant Crispld1 downregulation in heart biopsies of the heart-specific KO mouse line. There was a great variability of Crispld1 expression between the analyzed mice, suggesting that recombination was not completely successful in all animals. Additionally, heart-biopsies obtained from the left ventricle contain various types of heart cells, but Crispld1 is only deleted CM-specifically in this mouse line. Therefore, background mRNA influencing the result is expected. In order to minimize the background effect of other heart cell types than CMs, RT-qPCR analysis was additionally performed in isolated CMs of heart-specific KO 
mice, but again it was not possible to validate Crispld1 exon 5 deletion. However, due to the methodology of $\mathrm{CM}$-isolation, other cell types could not completely be eliminated and were therefore still influencing the result. Future experiments using for example FACS for specific CM sorting and subsequent RNA analysis might help to eliminate these background cells and therefore increase cell purity for a reliable result.

Many attempts using various primer combinations were performed to validate heart-specific Crispld1 KO. For example, primers that could only bind after successful deletion did not amplify significantly more product, probably due to high variabilities in the heart-specific KO mouse line and overall low Crispld1 expression (Appendix, Figure 5-2). As KO validation was not possible on protein level, due to a commercially unavailable antibody and as it was not detectable in WT mouse samples used for proteomics, this validation is completely based on DNA and RNA level missing the last step for complete verification.

However, various factors influenced the validation of the heart-specific KO mouse in RT-qPCR. There were great variabilities between Crispld1 expression in CDNA samples, presence of background cells and the efficiency of Cre-recombination in the different individuals which could also play a role. Unfortunately, during this thesis it was not possible to compare expression of Crispld1 in isolated CMs vs. non-CMs to measure the direct influence of background heart cells on the overall Crispld1 expression. As Crispld1 expression is overall low in left ventricles (see Appendix, Figure 5-3), a higher expression in non-CMs compared to CMs could have a great influence on the RT-qPCR. Therefore, this measurement could have helped to understand the discrepancy between the DNA and RNA KO validation and therefore should be performed in the future.

\subsubsection{Heart-specific Crispld1 KO but not ubiquitous Crispld1 KO leads to a dilated cardiac phenotype}

Although the KO could not successfully be proven in both $\mathrm{KO}$ mouse lines, both were used to examine the functional role of Crispld1 in the heart and compared to their controls. The ubiquitous Crispld1 KO line was analyzed up to one year of age. These mice showed normal and therefore comparable mortality rates to their controls. They had no serious phenotypic defects, although visual observations suggested a smaller body size. However, as the focus of this thesis was mainly on the heart, these observations were not further analyzed. In contrast, 
heart-specific KO mice had a significantly higher mortality compared to all other analyzed mouse lines. About $50 \%$ of these mice already died before the age of 225 days and none of them reached an age higher than 305 days. Previous studies reported, that the Crerecombinase activity of the Myh6-Cre line can lead to cardiotoxicity and cardiomyopathy by the age of 8-12 months (Davis et al., 2012) and therefore can influence the survival. Although the Myh6-Cre control line also showed a significant decreased survival compared to the flxcontrol and to the ubiquitous KO line, this survival was still significant increased compared to the heart-specific KO line underlining that this reduced mortality is not completely due to the Cre-recombinase. Therefore, results suggest that the heart-specific Crispld1 KO, but not the ubiquitous Crispld1 KO leads to an increased mortality and somehow influences the survival. In order to explain this outcome and to identify mechanisms that can lead to this severe mortality rate, echocardiographic analyses were performed. The ubiquitous KO mice did not show any abnormalities in their heart function in echocardiography at the age of 24 weeks, 32 weeks or one year. In contrast, heart-specific Crispld1 KO mice showed a reduced ejection fraction, reduced fractional shortening and increased left ventricular dimension at the age of 24 weeks which deteriorated at 32 weeks and could not be performed at one year of age due to early death. As expected, Myh6-Cre mice did also show a similar phenotype as the heartspecific Crispld1 KO mice, although this phenotype was less severe and showed only decreased EF and FS. Previous studies showed that Myh6-Cre mice develop an age-dependent cardiomyopathy starting at 6 months of age (Rehmani et al., 2019). However, the mechanism through which this cardiomyopathy is caused remains unclear, as two different phenotypes were found in the Myh6-Cre mouse line: hypertrophic cardiomyopathy (Pugach et al., 2015) and dilated cardiomyopathy (Rehmani et al., 2019). However, Myh6-Cre leads to DNA damage response in both phenotypes resulting in increased expression of myocyte death inducers and fibrosis. These molecular changes are nevertheless also influenced by various factors suggesting the distinct pathologies (Rehmani et al., 2019). Furthermore, levels of Cre recombinase influence this phenotype, thus low-expressing Cre can minimize off-target effects (Buerger et al., 2006; Koitabashi et al., 2009). For CMV-Cre mice, this phenotype was not published, neither do my results show any signs of cardiomyopathy. This suggests that the Cre-driver alone has a great influence on the phenotype. Therefore, an explanation for the severe heart-specific Crispld1 KO phenotype could be that Crispld1 somehow exacerbates the 
Myh6-Cre phenotype. This suggests that it accelerates the development of the dilated cardiomyopathy, probably by influencing the molecular changes due to DNA damage.

Nevertheless, our results showed that mice lacking Crispld1 heart-specific, but not globally, develop a dilated heart phenotype with reduced pump function, explaining the high mortality. This observation is surprising as the heart-specific KO could not be validated on mRNA level, whereas the ubiquitous KO mouse showed complete deletion of exon 5 of Crispld1 and downregulation of the remaining mRNA. One explanation for the difference between the heart-specific and ubiquitous KO mouse line could be a compensatory mechanism when Crispld1 is deleted globally, known as genetic robustness (Barbaric et al., 2007). A reason could be the use of alternative pathways, which was already identified for yeast. Depending on the physiological and environmental condition, their central metabolic pathway is ensured to function by using an alternative pathway under conditions where other pathways are not functional (Maltsev et al., 2005). These alternatives such as parallel pathways connecting regulators are rather found to be quite common to ensure survival (Wagner \& Wright, 2007). Possibly, a global deletion is necessary to activate these alternative pathways and could therefore be a reason for this significant difference between the heart-specific and the ubiquitous knockout line phenotype.

Our previous findings in iPSC-CMs suggested a beneficial effect of CRISPLD1 KO in the heart due to the rescue after toxin treatment. Therefore, the severe phenotype of the heart-specific KO mouse was surprising as rather the opposite was expected: CRISPLD1 KO preventing a severe heart failure phenotype. In addition to this expectation, it is not clear where this phenotype arises from due to the failure of successful KO validation.

The use of single-cell sequencing technology of isolated mouse CMs in the future could maybe help to answer this question and to possibly identify cells that could have performed successful recombination and therefore carry the deletion of Crispld1. These cells should be analyzed by their transcriptome to compare the data to the transcriptome of CRISPLD1 KO iPSC-CMs. This might help to elucidate mechanisms that differ in mouse and human after CRISPLD1 KO and furthermore, lead to the severe cardiac phenotype in mouse contrary to the beneficial effect of CRISPLD1 loss-of-function in iPSC-CMs. 


\subsubsection{Molecular analyses cannot explain the dilated heart-phenotype}

To get insights into molecular changes that occur in the KO mice and to further characterize the resulting cardiac phenotype to find an explanation, molecular and histological analyses were performed. Interestingly, the minimal fiber diameter and its size distribution was not changed in any mouse line, suggesting that the CMs do not develop hypertrophy. This is in line with the observation from RT-qPCR analyses. Nppa and Nppb, both hypertrophic stress markers, were only upregulated when compared to the flx-control line but not to the Crecontrols, indicating that any regulation is due to the Cre-recombinase background (Davis et al., 2012) and therefore not a consequence of the Crispld1 KO. Myh6, encoding $\alpha-M y H C$, and Myh7, encoding ß-MyHC, are known to be downregulated or upregulated in failing hearts, respectively (Lowes et al., 1997) and therefore chosen for analysis. The CRISPLD1 KO iPSC-CMs datasets also show a significantly reduced expression of Myh6 compared to isogenic controls. However, both genes were not significantly regulated in the mouse models. Another important gene in the failing heart is Atp2a2 which encodes SERCA2a. Its expression and activity is reduced upon heart failure affecting calcium signaling in the heart (Eisner et al., 2013). This leads to a longer calcium decay and decreased calcium amplitude (Eisner et al., 2013) contrary to the findings after Crispld1 KO in iPSC-CMs. However, Atp2a2 was not regulated in the mouse models. Therefore, RT-qPCR analysis of common genes that are known to play a role in heart failure and were regulated in our iPSC-CM model could not explain the findings in echocardiography on the molecular level.

Another molecule that plays a central role in the pathological hypertrophic process is the Camk2a. Expression and activity are increased in the failing heart and in several animal models of hypertrophy and heart failure (Anderson, 2009). It is involved in disturbances of the calcium homeostasis in $\mathrm{HF}$ by phosphorylating the ryanodine receptor and phospholamban (Zhang et al., 2003; Ferrero et al., 2007). Additionally, CM-specific overexpression of Camk2a in mice was shown to induce hypertrophy transitioning to dilated cardiomyopathy with disturbed calcium homeostasis, ventricular dysfunction and early death (Zhang et al., 2003). Therefore, I analyzed the activity of Camk2a in both $\mathrm{KO}$ mouse lines. The results indicated, that Camk2a activity is not changed in the ubiquitous $\mathrm{KO}$ mouse line but might be more active in the heartspecific KO mice due to its increased phosphorylation status. Although this observation was not significant when heart-specific KO mice were compared to the Myh6-Cre mouse line, but 
only to the flx-control, the tendency is similar and therefore a good hint for suggesting an increased activity. Big variations, especially between Myh6-Cre mice influence this outcome and therefore the statistic result. Nevertheless, this result could give at least a first idea about a pathway modification that can explain the severe dilated heart phenotype after heartspecific Crispld1 KO.

Fibrosis is one of the maladaptive changes in pathological cardiac hypertrophy when myocardial growth occurs and can lead to the development of HF (Oka \& Komuro, 2008; Nakamura \& Sadoshima, 2018). Therefore, fibrotic areas were analyzed by Picro Sirius Red Staining, but did not show any significant differences between the mouse lines. However, one reason for this outcome can be the small number of mice used for analysis together with great variances between the individuals which could mask a statistically relevant effect. Especially, the heart-specific KO mouse and the Myh6-Cre control line showed huge variations between individual mice and therefore influence the statistics. These variations can be due to several issues. Apart from the efficiency of the Cre-recombinase to induce the heart-specific CRISPLD1 KO, factors like sex, age, body weight, and environmental factors and interactions between mice might have an influence on experimental outcomes (Howard, 2002). Therefore, it cannot be completely excluded, that fibrosis occurs in the heart of the KO mouse line, especially the heart-specific KO line.

Altogether, the analyses of RNA expression, Camk2a protein expression, fibrosis, and fiber diameter could not unravel the molecular events occurring in the heart after the heart-specific Crispld1 KO to explain the dilated heart-phenotype. Although some of these experiments showed very small variances suggesting that the results are valid, others had great variabilities between individuals and could therefore mask possible effects. Repetition of experiments with increasing individuals could help to validate the results and to exclude misinterpretation to possibly identify any molecular pathway in the KO mouse that is influenced or regulated by Crispld1 and lead to the severe heart phenotype.

\subsubsection{Crispld1 KO in mice does not influence the cardiac calcium homeostasis}

Analysis of the calcium transients in CRISPLD1 KO iPSC-CMs suggests that the gene negatively regulates calcium cycling due to the increased amplitude and rise time together with the decreased calcium decay after CRISPLD1 loss-of-function. To compare this result to the mouse 
model and to verify if results obtained in human cell culture can be further proved in the murine system, calcium transients of isolated mouse CMs were measured. In the mouse models, significant differences in the ubiquitous CRISPLD1 KO mouse were observed for the amplitude at $1 \mathrm{~Hz}$ and the CaT decay 50 at $2 \mathrm{~Hz}$ and $4 \mathrm{~Hz}$ and in the heart-specific $\mathrm{KO}$ mouse for the amplitude at $4 \mathrm{~Hz}$, but both compared to the flx control, not to their Cre control lines. Instead, the Cre control lines also showed partially significant differences compared to the flx control line. This suggests that the results might be due to the Cre-background and should therefore be interpreted with care. Although, the number of analyzed cells is between 16 and 32 , the number of individuals the CMs were isolated from was only 1-2 mice due to experimental difficulties. In addition, the tendencies observed in the transients of mouse CMs comparing the knockout line and the flx line are not completely in line with the results obtained in iPSC-CMs. Although the amplitude is increased, similar to results found in iPSCCMs, the CaT decay 50 is also increased, contrary to the findings in iPSC-CMs. In HF, calcium transients normally show a reduced amplitude and slower decay, due to abnormal SR function with reduced SR calcium storage (Piacentino et al., 2003; Sankaranarayanan et al., 2016). Whereas in the iPSC-CM model CRISPLD1 KO led to beneficial effects, more specifically an increased amplitude and faster decay, contrary to HF events, findings in the heart-specific KO mouse model seem to be harmful, resulting in a severe cardiac phenotype but could not be explained by the calcium transient data. As the calcium changes were only observed in the KO mouse models in comparison to the flx control line, not to the Cre controls, no conclusion can be made. It is therefore essential to repeat the analysis and increase the number of mice to make sure that effects will not be masked by the small number of animals and the varieties between individuals that can influence the outcome (Howard, 2002).

\subsection{Summary and outlook}

In our study, CRISPLD1 was first described and characterized in the cardiac context and more specifically, the context of heart diseases by the use of a human iPSC model and a murine KO model system.

With the use of the CRISPR/Cas9 system in the iPSCs, I was able to identify CRISPLD1 as a negative regulator in the calcium homeostasis in iPSC-CMs although I could not elucidate its specific function, localization or mechanism. In short, the main cell culture findings presented in this thesis are: 
- CRISPLD1 KO does not influence the differentiation efficiency or the CM sarcomeric structure

- CRISPLD1 KO leads to an increased calcium transient amplitude and rise time, but a faster CaT decay suggesting an increased systolic calcium level

- CRISPLD1 KO can rescue the negative effects of the toxin helothermine, a known calcium blocker, on the calcium homeostasis

- CRISPLD1 is involved in regulation of cardiomyopathy, inflammation, calcium homeostasis and ECM organization

Future cell culture studies should address questions regarding intracellular and SR $\mathrm{Ca}^{2+}$-load and the role of $\mathrm{Ca}^{2+}$-currents to validate the obtained results. Additionally, localization studies might help to elucidate the target site in order to identify the exact mechanism of CRISPLD1.

By using the Cre/loxP system in mouse, two mouse lines were generated, a heart-specific Crispld1 KO line and an ubiquitous Crispld1 KO line. In these mouse lines, a severe cardiac phenotype for the heart-specific KO mouse line was observed, but not for the ubiquitous KO line, although KO validation was only successful for the latter. The main findings from the mouse models are:

- Heart-specific Crispld1 recombinase results in a dilated cardiomyopathy with reduced survival although Crispld1 deletion could not be validated completely

- Ubiquitous Crispld1 deletion does not cause a mouse phenotype and therefore seems to be compensated by unknown mechanisms

- Heart-specific Crispld1 KO, but not ubiquitous Crispld1 KO enhances Camk2a activity

- Crispld1 KO does not induce a CM hypertrophic phenotype neither alter hypertrophic marker expression or increase fibrosis

- Crispld1 seems not to alter the mouse CM calcium homeostasis

In order to validate the findings in the murine model system, it is essential to find a way to validate the heart-specific Crispld1 KO, possibly by FACS to eliminate background cells in analysis or by single-cell sequencing. Especially single-cell sequencing could furthermore identify specific targets that were modified by Crispld1 $\mathrm{KO}$ and explain the severe phenotype. Comparison to transcriptomics of the ubiquitous mouse line might help to identify the reason for the different phenotype when comparing heart-specific vs. global Crispld1 KO and to the 
results obtained in iPSC experiments. Apart from that, it is essential to increase the number of individuals used for experiments as great variabilities in between are known to influence the outcome and could mask possible effects.

In the future, much effort has to be made to validate and understand the results of this thesis. Although results obtained in iPSC-CMs seemed promising, the findings in the mouse model could not confirm them and are partially contradictory. However, many technical difficulties occurred in the mouse models and therefore influenced the final results. To finally make a conclusion about the exact role and importance of CRISPLD1 in the human heart, it is essential to identify its specific mechanism and potential targets.

This thesis gives first insights into the potential function and importance of the priorly uncharacterized CRISPDL1 in the context of heart disease studies in a murine and human model system and paved the way for future research regarding specific cardiac CRISPLD1 function. 


\section{Appendix}

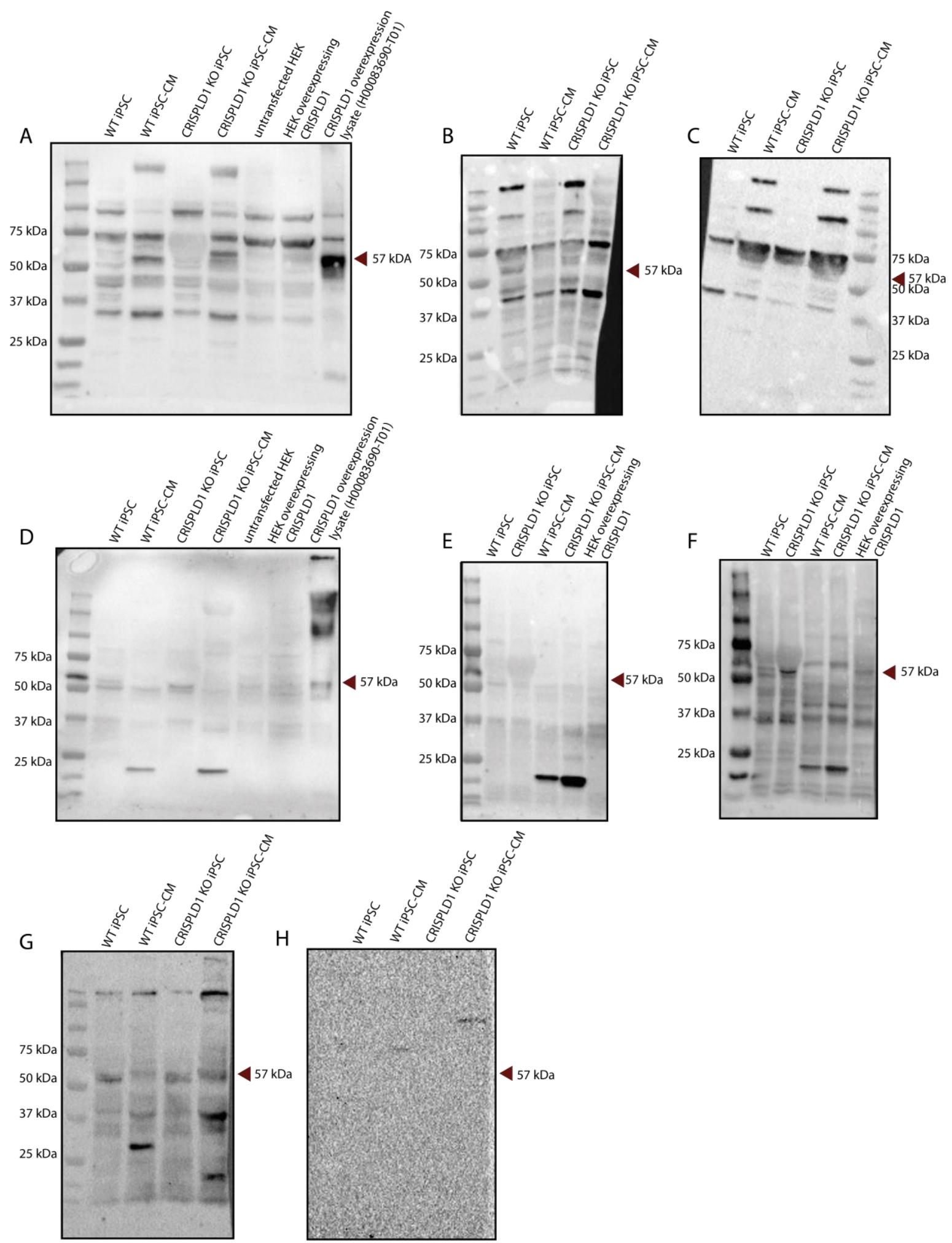

Figure 5-1 Western Blot analysis of four commercially available CRISPLD1 antibodies. WT and KO iPSCS and $\mathrm{CMs}$, transfected and untransfected HEK cells (70 $\mathrm{mg}$ total protein each) and CRISPLD1 overexpression lysate (H00083690-T01) were investigated. The red arrow shows the expected protein size of 57 kDA. A) abx301729; diluted 1:500. $5 \%$ milk served as blocking solution. B) HPA024725; diluted 1:250. $5 \%$ BSA served as blocking solution. C) HPA024725; diluted 1:250. 5 \% milk served as blocking solution. D) H00083690-B01P; diluted 1:500, $5 \%$ milk served as blocking solution. E) H00083690-B01P; diluted 1:500. 5\% BSA served as blocking solution. F) 
H00083690-B01P; diluted 1:500. 5 \% milk serves as blocking solution. G) ab123039; diluted 1:1000. 5 \% BSA served as blocking solution. H) ab123039; diluted 1:1000. $5 \%$ milk served as blocking solution. WT cells, transfected HEK and the lysate were used as the positive control, KO cells and untransfected HEK as the negative control.

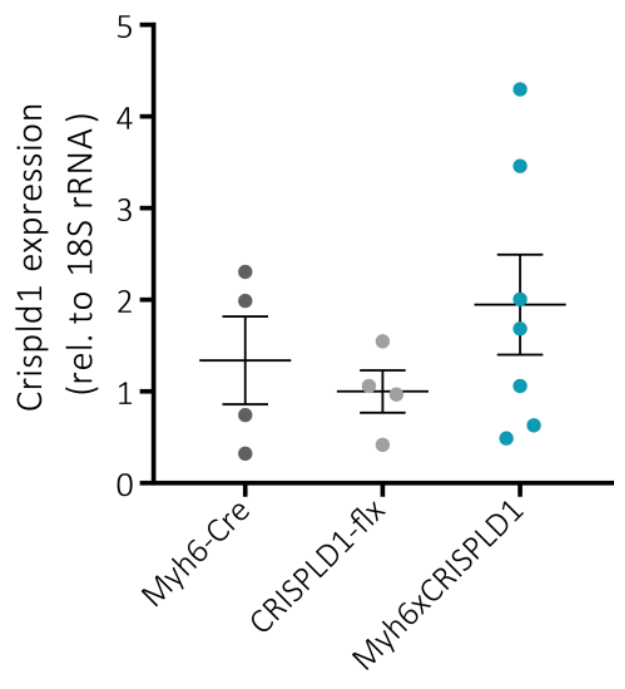

Figure 5-2 Validation of Myh6xCRISPLD1 KO mice by RT-qPCR. Although primers could only bind when recombination was successful (Primer ms_CRISPLD1_ex4-6_3), there was no significant difference between controls and the Myh6xCRISPLD1 mouse. $n=4-7$. Statistics were performed with an unpaired two-tailed t-test. Error bars indicate SD.

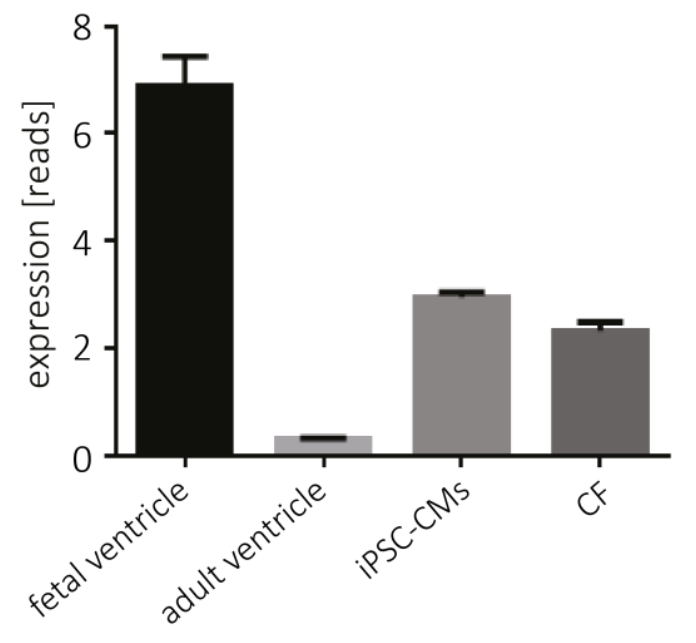

Figure 5-3 CRISPLD1 expression reads in human fetal and adult ventricle, iPSC-CMs and cardiac fibroblasts (CF). Expression was determined by RNA-sequencing given as reads per kilobase per million mapped reads (rpkm). Adapted from (Khadjeh et al., 2020). 


\section{References}

Adams, J.W., Sakata, Y., Davis, M.G., Sah, V.P., Wang, Y., Liggett, S.B., Chien, K.R., Brown, J.H. \& Dorn, G.W. (1998) Enhanced Galphaq signaling: a common pathway mediates cardiac hypertrophy and apoptotic heart failure. Proceedings of the National Academy of Sciences of the United States of America, 95, 10140-10145.

Agah, R., Frenkel, P.A., French, B.A., Michael, L.H., Overbeek, P.A. \& Schneider, M.D. (1997) Gene recombination in postmitotic cells. Targeted expression of Cre recombinase provokes cardiac-restricted, site-specific rearrangement in adult ventricular muscle in vivo. The Journal of clinical investigation, 100, 169-179.

Ai, X., Curran, J.W., Shannon, T.R., Bers, D.M. \& Pogwizd, S.M. (2005) Ca2+/calmodulindependent protein kinase modulates cardiac ryanodine receptor phosphorylation and sarcoplasmic reticulum Ca2+ leak in heart failure. Circulation research, 97, 1314-1322.

Akin, B.L., Hurley, T.D., Chen, Z. \& Jones, L.R. (2013) The structural basis for phospholamban inhibition of the calcium pump in sarcoplasmic reticulum. The Journal of biological chemistry, 288, 30181-30191.

Anderson, M.E. (2009) CaMKII and a failing strategy for growth in heart. The Journal of clinical investigation, 119, 1082-1085.

Arstall, M.A., Sawyer, D.B., Fukazawa, R. \& Kelly, R.A. (1999) Cytokine-mediated apoptosis in cardiac myocytes: the role of inducible nitric oxide synthase induction and peroxynitrite generation. Circulation research, 85, 829-840.

Ather, S., Respress, J.L., Li, N. \& Wehrens, X.H.T. (2013) Alterations in ryanodine receptors and related proteins in heart failure. Biochimica et biophysica acta, 1832, 2425-2431.

Barbaric, I., Miller, G. \& Dear, T.N. (2007) Appearances can be deceiving: phenotypes of knockout mice. Briefings in functional genomics \& proteomics, 6, 91-103.

Bengel, P., Ahmad, S., Tirilomis, P., Trum, M., Dybkova, N., Wagner, S., Maier, L.S., Hasenfuß, G. \& Sossalla, S. (2020) Contribution of the neuronal sodium channel NaV1.8 to sodiumand calcium-dependent cellular proarrhythmia. Journal of molecular and cellular cardiology, 144, 35-46.

Bentivegna, L.A., Ablin, L.W., Kihara, Y. \& Morgan, J.P. (1991) Altered calcium handling in left ventricular pressure-overload hypertrophy as detected with aequorin in the isolated, perfused ferret heart. Circulation research, 69, 1538-1545.

Bers, D.M. \& Morotti, S. (2014) Ca(2+) current facilitation is CaMKII-dependent and has arrhythmogenic consequences. Frontiers in pharmacology, 5, 144.

Beuckelmann, D.J., Näbauer, M. \& Erdmann, E. (1992) Intracellular calcium handling in isolated ventricular myocytes from patients with terminal heart failure. Circulation, 85, 1046-1055. 
Bindea, G., Mlecnik, B., Hackl, H., Charoentong, P., Tosolini, M., Kirilovsky, A., Fridman, W.H., Pagès, F., Trajanoski, Z. \& Galon, J. (2009) ClueGO: a Cytoscape plug-in to decipher functionally grouped gene ontology and pathway annotation networks. Bioinformatics (Oxford, England), 25, 1091-1093.

Braunwald, E. \& Chidsey, C.A. (1965) The adrenergic nervous system in the control of the normal and failing heart. Proceedings of the Royal Society of Medicine, 58, 1063-1066.

Buerger, A., Rozhitskaya, O., Sherwood, M.C., Dorfman, A.L., Bisping, E., Abel, E.D., Pu, W.T., Izumo, S. \& Jay, P.Y. (2006) Dilated cardiomyopathy resulting from high-level myocardial expression of Cre-recombinase. Journal of cardiac failure, 12, 392-398.

Chen, X., Piacentino, V., Furukawa, S., Goldman, B., Margulies, K.B. \& Houser, S.R. (2002) Ltype $\mathrm{Ca} 2+$ channel density and regulation are altered in failing human ventricular myocytes and recover after support with mechanical assist devices. Circulation research, 91, 517-524.

Chiquet, B.T., Henry, R., Burt, A., Mulliken, J.B., Stal, S., Blanton, S.H. \& Hecht, J.T. (2011) Nonsyndromic cleft lip and palate: CRISPLD genes and the folate gene pathway connection. Birth defects research. Part A, Clinical and molecular teratology, 91, 44-49.

Cigola, E., Kajstura, J., Li, B., Meggs, L.G. \& Anversa, P. (1997) Angiotensin II activates programmed myocyte cell death in vitro. Experimental cell research, 231, 363-371.

Cyganek, L., Tiburcy, M., Sekeres, K., Gerstenberg, K., Bohnenberger, H., Lenz, C., Henze, S., Stauske, M., Salinas, G., Zimmermann, W.-H., Hasenfuss, G. \& Guan, K. (2018) Deep phenotyping of human induced pluripotent stem cell-derived atrial and ventricular cardiomyocytes. JCl Insight, 3.

Davis, J., Maillet, M., Miano, J.M. \& Molkentin, J.D. (2012) Lost in transgenesis: a user's guide for genetically manipulating the mouse in cardiac research. Circulation research, 111, 761-777.

Del Monte, F., Harding, S.E., Schmidt, U., Matsui, T., Kang, Z.B., Dec, G.W., Gwathmey, J.K., Rosenzweig, A. \& Hajjar, R.J. (1999) Restoration of contractile function in isolated cardiomyocytes from failing human hearts by gene transfer of SERCA2a. Circulation, 100, 2308-2311.

Dickstein, K., Cohen-Solal, A., Filippatos, G., McMurray, J.J.V., Ponikowski, P., Poole-Wilson, P.A., Strömberg, A., van Veldhuisen, D.J., Atar, D., Hoes, A.W., Keren, A., Mebazaa, A., Nieminen, M., Priori, S.G. \& Swedberg, K. (2008) ESC guidelines for the diagnosis and treatment of acute and chronic heart failure 2008: the Task Force for the diagnosis and treatment of acute and chronic heart failure 2008 of the European Society of Cardiology. Developed in collaboration with the Heart Failure Association of the ESC (HFA) and endorsed by the European Society of Intensive Care Medicine (ESICM). European journal of heart failure, 10, 933-989. 
Dirkx, E., Da Costa Martins, P.A. \& Windt, L.J. de (2013) Regulation of fetal gene expression in heart failure. Biochimica et biophysica acta, 1832, 2414-2424.

Doenst, T., Nguyen, T.D. \& Abel, E.D. (2013) Cardiac metabolism in heart failure: implications beyond ATP production. Circulation research, 113, 709-724.

DuBridge, R.B., Tang, P., Hsia, H.C., Leong, P.M., Miller, J.H. \& Calos, M.P. (1987) Analysis of mutation in human cells by using an Epstein-Barr virus shuttle system. Mol. Cell. Biol., 7, 379-387.

Dybkova, N., Ahmad, S., Pabel, S., Tirilomis, P., Hartmann, N., Fischer, T.H., Bengel, P., Tirilomis, T., Ljubojevic, S., Renner, A., Gummert, J., Ellenberger, D., Wagner, S., Frey, N., Maier, L.S., Streckfuss-Bömeke, K., Hasenfuss, G. \& Sossalla, S. (2018) Differential regulation of sodium channels as a novel proarrhythmic mechanism in the human failing heart. Cardiovascular Research, 114, 1728-1737.

Eisner, D., Caldwell, J. \& Trafford, A. (2013) Sarcoplasmic reticulum Ca-ATPase and heart failure 20 years later. Circulation research, 113, 958-961.

Eisner, D.A., Caldwell, J.L., Kistamás, K. \& Trafford, A.W. (2017) Calcium and ExcitationContraction Coupling in the Heart. Circulation research, 121, 181-195.

El-Battrawy, I., Lan, H., Cyganek, L., Zhao, Z., Li, X., Buljubasic, F., Lang, S., Yücel, G., Sattler, K., Zimmermann, W.-H., Utikal, J., Wieland, T., Ravens, U., Borggrefe, M., Zhou, X.-B. \& Akin, I. (2018) Modeling Short QT Syndrome Using Human-Induced Pluripotent Stem CellDerived Cardiomyocytes. Journal of the American Heart Association, 7.

Ferrero, P., Said, M., Sánchez, G., Vittone, L., Valverde, C., Donoso, P., Mattiazzi, A. \& Mundiña-Weilenmann, C. (2007) Ca2+/calmodulin kinase II increases ryanodine binding and $\mathrm{Ca} 2+$-induced sarcoplasmic reticulum $\mathrm{Ca} 2+$ release kinetics during $\beta$-adrenergic stimulation. Journal of molecular and cellular cardiology, 43, 281-291.

Frey, N., McKinsey, T.A. \& Olson, E.N. (2000) Decoding calcium signals involved in cardiac growth and function. Nature medicine, 6, 1221-1227.

Frey, N. \& Olson, E.N. (2003) Cardiac hypertrophy: the good, the bad, and the ugly. Annual review of physiology, 65, 45-79.

Frost, R.J.A. \& Engelhardt, S. (2007) A secretion trap screen in yeast identifies protease inhibitor 16 as a novel antihypertrophic protein secreted from the heart. Circulation, 116, 1768-1775.

Gao, Q., Wang, P., Wu, Z., Qiu, H., Lin, B., Chen, J., Cen, J. \& Zhuang, J. (2020) Angiotensin II induces apoptosis in human induced pluripotent stem cell-derived cardiomyocytes.

Ghaemmaghami, S., Huh, W.-K., Bower, K., Howson, R.W., Belle, A., Dephoure, N., O'Shea, E.K. \& Weissman, J.S. (2003) Global analysis of protein expression in yeast. Nature, 425, 737-741. 
Gibbs, G.M., Roelants, K. \& O'Bryan, M.K. (2008) The CAP superfamily: cysteine-rich secretory proteins, antigen 5 , and pathogenesis-related 1 proteins--roles in reproduction, cancer, and immune defense. Endocrine reviews, 29, 865-897.

Gibbs, G.M., Scanlon, M.J., Swarbrick, J., Curtis, S., Gallant, E., Dulhunty, A.F. \& O'Bryan, M.K. (2006) The cysteine-rich secretory protein domain of Tpx-1 is related to ion channel toxins and regulates ryanodine receptor Ca2+ signaling. The Journal of biological chemistry, 281, 4156-4163.

Gonzalez-Begne, M., Lu, B., Han, X., Hagen, F.K., Hand, A.R., Melvin, J.E. \& Yates, J.R. (2009) Proteomic analysis of human parotid gland exosomes by multidimensional protein identification technology (MudPIT). Journal of proteome research, 8, 1304-1314.

Goonasekera, S.A., Hammer, K., Auger-Messier, M., Bodi, I., Chen, X., Zhang, H., Reiken, S., Elrod, J.W., Correll, R.N., York, A.J., Sargent, M.A., Hofmann, F., Moosmang, S., Marks, A.R., Houser, S.R., Bers, D.M. \& Molkentin, J.D. (2012) Decreased cardiac L-type Ca ${ }^{2+}$ channel activity induces hypertrophy and heart failure in mice. The Journal of clinical investigation, 122, 280-290.

Grossman, W., Jones, D. \& McLaurin, L.P. (1975) Wall stress and patterns of hypertrophy in the human left ventricle. The Journal of clinical investigation, 56, 56-64.

Hajar, R. (2019) Congestive Heart Failure: A History. Heart views : the official journal of the Gulf Heart Association, 20, 129-132.

Harsdorf, R. von, Li, P.F. \& Dietz, R. (1999) Signaling pathways in reactive oxygen speciesinduced cardiomyocyte apoptosis. Circulation, 99, 2934-2941.

Heineke, J. \& Molkentin, J.D. (2006) Regulation of cardiac hypertrophy by intracellular signalling pathways. Nature reviews. Molecular cell biology, 7, 589-600.

Hoch, B., Meyer, R., Hetzer, R., Krause, E.G. \& Karczewski, P. (1999) Identification and expression of delta-isoforms of the multifunctional $\mathrm{Ca} 2+$ /calmodulin-dependent protein kinase in failing and nonfailing human myocardium. Circulation research, 84, 713-721.

Holmfeldt, P., Ganuza, M., Marathe, H., He, B., Hall, T., Kang, G., Moen, J., Pardieck, J., Saulsberry, A.C., Cico, A., Gaut, L., McGoldrick, D., Finkelstein, D., Tan, K. \& McKinneyFreeman, S. (2016) Functional screen identifies regulators of murine hematopoietic stem cell repopulation. The Journal of experimental medicine, 213, 433-449.

Howard, B.R. (2002) Control of variability. ILAR journal, 43, 194-201.

Inamdar, A.A. \& Inamdar, A.C. (2016) Heart Failure: Diagnosis, Management and Utilization. Journal of clinical medicine, 5.

Kajstura, J., Cigola, E., Malhotra, A., Li, P., Cheng, W., Meggs, L.G. \& Anversa, P. (1997) Angiotensin II induces apoptosis of adult ventricular myocytes in vitro. Journal of molecular and cellular cardiology, 29, 859-870. 
Khadjeh, S., Hindmarsh, V., Weber, F., Cyganek, L., Vidal, R.O., Torkieh, S., StreckfussBömeke, K., Lbik, D., Tiburcy, M., Mohamed, B.A., Bonn, S., Toischer, K. \& Hasenfuss, G. (2020) CRISPLD1: a novel conserved target in the transition to human heart failure. Basic research in cardiology, 115, 27.

Kleinsorge, M. \& Cyganek, L. (2020) Subtype-Directed Differentiation of Human iPSCs into Atrial and Ventricular Cardiomyocytes. STAR protocols, 1, 100026.

Koitabashi, N., Bedja, D., Zaiman, A.L., Pinto, Y.M., Zhang, M., Gabrielson, K.L., Takimoto, E. \& Kass, D.A. (2009) Avoidance of transient cardiomyopathy in cardiomyocyte-targeted tamoxifen-induced MerCreMer gene deletion models. Circulation research, 105, 12-15.

Kostin, S., Pool, L., Elsässer, A., Hein, S., Drexler, H.C.A., Arnon, E., Hayakawa, Y., Zimmermann, R., Bauer, E., Klövekorn, W.-P. \& Schaper, J. (2003) Myocytes die by multiple mechanisms in failing human hearts. Circulation research, 92, 715-724.

Kranias, E.G. \& Hajjar, R.J. (2012) Modulation of cardiac contractility by the phospholamban/SERCA2a regulatome. Circulation research, 110, 1646-1660.

Kranz, A., Fu, J., Duerschke, K., Weidlich, S., Naumann, R., Stewart, A.F. \& Anastassiadis, K. (2010) An improved Flp deleter mouse in C57BI/6 based on Flpo recombinase. Genesis (New York, N.Y. : 2000), 48, 512-520.

Li, W., Wang, S., Qiu, C., Liu, Z., Zhou, Q., Kong, D., Ma, X. \& Jiang, J. (2019) Comprehensive bioinformatics analysis of acquired progesterone resistance in endometrial cancer cell line. Journal of translational medicine, 17, 58.

Li, X., Wu, N., Ji, H., Huang, Y., Hu, H., Li, J., Mi, S., Duan, S. \& Chen, X. (2020) A male-specific association between AGTR1 hypermethylation and coronary heart disease. Bosnian journal of basic medical sciences, 20, 31-36.

Li, Y., Li, X., Jia, N., Guo, S., Chu, S. \& Niu, W. (2013) Meta-analysis of the association between angiotensin II receptor, type 1 gene $\mathrm{A} 1166 \mathrm{C}$ polymorphism and coronary artery disease in Chinese populations. Journal of the renin-angiotensin-aldosterone system : JRAAS, 14, 82-90.

Liew, C.W., Xu, S., Wang, X., McCann, M., Whang Kong, H., Carley, A.C., Pang, J., Fantuzzi, G., O'Donnell, J.M. \& Lewandowski, E.D. (2017) Multiphasic Regulation of Systemic and Peripheral Organ Metabolic Responses to Cardiac Hypertrophy. Circulation. Heart failure, 10.

Lippi, G. \& Sanchis-Gomar, F. (2020) Global epidemiology and future trends of heart failure. AME Med J, 5, 15.

Lipskaia, L., Ly, H., Kawase, Y., Hajjar, R.J. \& Lompre, A.-M. (2007) Treatment of heart failure by calcium cycling gene therapy. Future cardiology, 3, 413-423.

Litviňuková, M., Talavera-López, C., Maatz, H., Reichart, D., Worth, C.L., Lindberg, E.L., Kanda, M., Polanski, K., Heinig, M., Lee, M., Nadelmann, E.R., Roberts, K., Tuck, L., 
Fasouli, E.S., DeLaughter, D.M., McDonough, B., Wakimoto, H., Gorham, J.M., Samari, S., Mahbubani, K.T., Saeb-Parsy, K., Patone, G., Boyle, J.J., Zhang, H., Zhang, H., Viveiros, A., Oudit, G.Y., Bayraktar, O.A., Seidman, J.G., Seidman, C.E., Noseda, M., Hubner, N. \& Teichmann, S.A. (2020) Cells of the adult human heart. Nature, 588, 466-472.

Lowes, B.D., Minobe, W., Abraham, W.T., Rizeq, M.N., Bohlmeyer, T.J., Quaife, R.A., Roden, R.L., Dutcher, D.L., Robertson, A.D., Voelkel, N.F., Badesch, D.B., Groves, B.M., Gilbert, E.M. \& Bristow, M.R. (1997) Changes in gene expression in the intact human heart. Downregulation of alpha-myosin heavy chain in hypertrophied, failing ventricular myocardium. The Journal of clinical investigation, 100, 2315-2324.

Luo, M. \& Anderson, M.E. (2013) Mechanisms of altered $\mathrm{Ca}^{2+}$ handling in heart failure. Circulation research, 113, 690-708.

Maillet, M., van Berlo, J.H. \& Molkentin, J.D. (2013) Molecular basis of physiological heart growth: fundamental concepts and new players. Nature reviews. Molecular cell biology, 14, 38-48.

Maltsev, N., Glass, E.M., Ovchinnikova, G. \& Gu, Z. (2005) Molecular mechanisms involved in robustness of yeast central metabolism against null mutations. Journal of biochemistry, 137, 177-187.

McMullen, J.R. \& Jennings, G.L. (2007) Differences between pathological and physiological cardiac hypertrophy: novel therapeutic strategies to treat heart failure. Clinical and experimental pharmacology \& physiology, 34, 255-262.

Medvedev, R.Y., Sanchez-Alonso, J.L., Mansfield, C.A., Judina, A., Francis, A.J., Pagiatakis, C., Trayanova, N., Glukhov, A.V., Miragoli, M., Faggian, G. \& Gorelik, J. (2021) Local hyperactivation of L-type $\mathrm{Ca} 2+$ channels increases spontaneous $\mathrm{Ca} 2+$ release activity and cellular hypertrophy in right ventricular myocytes from heart failure rats. Scientific reports, 11, 4840 .

Mercadier, J.J., Lompré, A.M., Duc, P., Boheler, K.R., Fraysse, J.B., Wisnewsky, C., Allen, P.D., Komajda, M. \& Schwartz, K. (1990) Altered sarcoplasmic reticulum Ca2(+)-ATPase gene expression in the human ventricle during end-stage heart failure. The Journal of clinical investigation, 85, 305-309.

Mochca-Morales, J., Martin, B.M. \& Possani, L.D. (1990) Isolation and characterization of Helothermine, a novel toxin from Heloderma horridum horridum (Mexican beaded lizard) venom. Toxicon, 28, 299-309.

Molkentin, J.D., Lu, J.-R., Antos, C.L., Markham, B., Richardson, J., Robbins, J., Grant, S.R. \& Olson, E.N. (1998) A Calcineurin-Dependent Transcriptional Pathway for Cardiac Hypertrophy. Cell, 93, 215-228.

Morrissette, J., Krätzschmar, J., Haendler, B., el-Hayek, R., Mochca-Morales, J., Martin, B.M., Patel, J.R., Moss, R.L., Schleuning, W.D. \& Coronado, R. (1995) Primary structure and 
properties of helothermine, a peptide toxin that blocks ryanodine receptors. Biophysical Journal, 68, 2280-2288.

Nakamura, M. \& Sadoshima, J. (2018) Mechanisms of physiological and pathological cardiac hypertrophy. Nature reviews. Cardiology, 15, 387-407.

Nakayama, H., Chen, X., Baines, C.P., Klevitsky, R., Zhang, X., Zhang, H., Jaleel, N., Chua, B.H.L., Hewett, T.E., Robbins, J., Houser, S.R. \& Molkentin, J.D. (2007) Ca2+- and mitochondrial-dependent cardiomyocyte necrosis as a primary mediator of heart failure. The Journal of clinical investigation, 117, 2431-2444.

Neubauer, S. (2007) The failing heart--an engine out of fuel. The New England journal of medicine, 356, 1140-1151.

Nobile, M., Magnelli, V., Lagostena, L., Mochca-Morales, J., Possani, L.D. \& Prestipino, G. (1994) The toxin helothermine affects potassium currents in newborn rat cerebellar granule cells. The Journal of membrane biology, 139, 49-55.

Nobile, M., Noceti, F., Prestipino, G. \& Possani, L.D. (1996) Helothermine, a lizard venom toxin, inhibits calcium current in cerebellar granules. Experimental brain research, 110, 15-20.

O'Connell, T.D., Swigart, P.M., Rodrigo, M.C., Ishizaka, S., Joho, S., Turnbull, L., Tecott, L.H., Baker, A.J., Foster, E., Grossman, W. \& Simpson, P.C. (2006) Alpha1-adrenergic receptors prevent a maladaptive cardiac response to pressure overload. The Journal of clinical investigation, 116, 1005-1015.

Oka, T. \& Komuro, I. (2008) Molecular mechanisms underlying the transition of cardiac hypertrophy to heart failure. Circulation journal : official journal of the Japanese Circulation Society, 72 Suppl A, A13-6.

Osadchii, O.E. (2007) Cardiac hypertrophy induced by sustained beta-adrenoreceptor activation: pathophysiological aspects. Heart failure reviews, 12, 66-86.

Pacanowski, M.A., Gong, Y., Cooper-Dehoff, R.M., Schork, N.J., Shriver, M.D., Langaee, T.Y., Pepine, C.J. \& Johnson, J.A. (2008) beta-adrenergic receptor gene polymorphisms and beta-blocker treatment outcomes in hypertension. Clinical pharmacology and therapeutics, 84, 715-721.

Paradis, A.N., Gay, M.S. \& Zhang, L. (2014) Binucleation of cardiomyocytes: the transition from a proliferative to a terminally differentiated state. Drug discovery today, $19,602-$ 609.

Parikh, K.S., Fiuzat, M., Davis, G., Neely, M., Blain-Nelson, P., Whellan, D.J., Abraham, W.T., Adams, K.F., Felker, G.M., Liggett, S.B., O'Connor, C.M. \& Bristow, M.R. (2018) Dose Response of $\beta$-Blockers in Adrenergic Receptor Polymorphism Genotypes. Circulation. Genomic and precision medicine, 11, e002210. 
Piacentino, V., Weber, C.R., Chen, X., Weisser-Thomas, J., Margulies, K.B., Bers, D.M. \& Houser, S.R. (2003) Cellular basis of abnormal calcium transients of failing human ventricular myocytes. Circulation research, 92, 651-658.

Pierce, K.L., Premont, R.T. \& Lefkowitz, R.J. (2002) Seven-transmembrane receptors. Nature reviews. Molecular cell biology, 3, 639-650.

Pistner, A., Belmonte, S., Coulthard, T. \& Blaxall, B. (2010) Murine echocardiography and ultrasound imaging. Journal of visualized experiments : JoVE.

Ponikowski, P., Voors, A.A., Anker, S.D., Bueno, H., Cleland, J.G.F., Coats, A.J.S., Falk, V., González-Juanatey, J.R., Harjola, V.-P., Jankowska, E.A., Jessup, M., Linde, C., Nihoyannopoulos, P., Parissis, J.T., Pieske, B., Riley, J.P., Rosano, G.M.C., Ruilope, L.M., Ruschitzka, F., Rutten, F.H. \& van der Meer, P. (2016) 2016 ESC Guidelines for the diagnosis and treatment of acute and chronic heart failure: The Task Force for the diagnosis and treatment of acute and chronic heart failure of the European Society of Cardiology (ESC)Developed with the special contribution of the Heart Failure Association (HFA) of the ESC. European heart journal, 37, 2129-2200.

Principe, S., Jones, E.E., Kim, Y., Sinha, A., Nyalwidhe, J.O., Brooks, J., Semmes, O.J., Troyer, D.A., Lance, R.S., Kislinger, T. \& Drake, R.R. (2013) In-depth proteomic analyses of exosomes isolated from expressed prostatic secretions in urine. Proteomics, 13, 16671671.

Pugach, E.K., Richmond, P.A., Azofeifa, J.G., Dowell, R.D. \& Leinwand, L.A. (2015) Prolonged Cre expression driven by the $\alpha$-myosin heavy chain promoter can be cardiotoxic. Journal of molecular and cellular cardiology, 86, 54-61.

Rehmani, T., Salih, M. \& Tuana, B.S. (2019) Cardiac-Specific Cre Induces Age-Dependent Dilated Cardiomyopathy (DCM) in Mice. Molecules (Basel, Switzerland), 24.

Reuter, H. (1983) Calcium channel modulation by neurotransmitters, enzymes and drugs. Nature, 301, 569-574.

Ritchie, R.H. \& Delbridge, L.M.D. (2006) Cardiac hypertrophy, substrate utilization and metabolic remodelling: cause or effect? Clinical and experimental pharmacology \& physiology, 33, 159-166.

Rueden, C.T., Schindelin, J., Hiner, M.C., DeZonia, B.E., Walter, A.E., Arena, E.T. \& Eliceiri, K.W. (2017) ImageJ2: ImageJ for the next generation of scientific image data. BMC Bioinformatics, 18.

Sag, C.M., Wadsack, D.P., Khabbazzadeh, S., Abesser, M., Grefe, C., Neumann, K., Opiela, M.K., Backs, J., Olson, E.N., Brown, J.H., Neef, S., Maier, S.K.G. \& Maier, L.S. (2009) Calcium/calmodulin-dependent protein kinase II contributes to cardiac arrhythmogenesis in heart failure. Circulation. Heart failure, 2, 664-675. 
Salazar, N.C., Chen, J. \& Rockman, H.A. (2007) Cardiac GPCRs: GPCR signaling in healthy and failing hearts. Biochimica et biophysica acta, 1768, 1006-1018.

Sankaranarayanan, R., Li, Y., Greensmith, D.J., Eisner, D.A. \& Venetucci, L. (2016) Biphasic decay of the Ca transient results from increased sarcoplasmic reticulum Ca leak. The Journal of physiology, 594, 611-623.

Schindelin, J., Arganda-Carreras, I., Frise, E., Kaynig, V., Longair, M., Pietzsch, T., Preibisch, S., Rueden, C., Saalfeld, S., Schmid, B., Tinevez, J.-Y., White, D.J., Hartenstein, V., Eliceiri, K., Tomancak, P. \& Cardona, A. (2012) Fiji: an open-source platform for biological-image analysis. Nature methods, 9, 676-682.

Schwenk, F., Baron, U. \& Rajewsky, K. (1995) A cre-transgenic mouse strain for the ubiquitous deletion of loxP-flanked gene segments including deletion in germ cells. Nucleic acids research, 23, 5080-5081.

Shannon, P., Markiel, A., Ozier, O., Baliga, N.S., Wang, J.T., Ramage, D., Amin, N., Schwikowski, B. \& Ideker, T. (2003) Cytoscape: a software environment for integrated models of biomolecular interaction networks. Genome research, 13, 2498-2504.

Shiels, H.A. (2011) DESIGN AND PHYSIOLOGY OF THE HEART | Cardiac Excitation-Contraction Coupling: Routes of Cellular Calcium Flux. In , Encyclopedia of Fish Physiology. Elsevier, pp. 1045-1053.

Shin, J. \& Johnson, J.A. (2007) Pharmacogenetics of beta-blockers. Pharmacotherapy, 27, 874-887.

Singh, K., Communal, C., Sawyer, D.B. \& Colucci, W.S. (2000) Adrenergic regulation of myocardial apoptosis. Cardiovascular Research, 45, 713-719.

Skarnes, W.C., Rosen, B., West, A.P., Koutsourakis, M., Bushell, W., Iyer, V., Mujica, A.O., Thomas, M., Harrow, J., Cox, T., Jackson, D., Severin, J., Biggs, P., Fu, J., Nefedov, M., Jong, P.J. de, Stewart, A.F. \& Bradley, A. (2011) A conditional knockout resource for the genome-wide study of mouse gene function. Nature, 474, 337-342.

Sousa Abreu, R. de, Penalva, L.O., Marcotte, E.M. \& Vogel, C. (2009) Global signatures of protein and mRNA expression levels. Molecular bioSystems, 5, 1512-1526.

Stansfield, W.E., Ranek, M., Pendse, A., Schisler, J.C., Wang, S., Pulinilkunnil, T. \& Willis, M.S. (2014) The Pathophysiology of Cardiac Hypertrophy and Heart Failure. In , Cellular and Molecular Pathobiology of Cardiovascular Disease. Elsevier, pp. 51-78.

Sujit, K.M., Sarkar, S., Singh, V., Pandey, R., Agrawal, N.K., Trivedi, S., Singh, K., Gupta, G. \& Rajender, S. (2018) Genome-wide differential methylation analyses identifies methylation signatures of male infertility. Human reproduction (Oxford, England), 33, 2256-2267. 
Tham, Y.K., Bernardo, B.C., Ooi, J.Y.Y., Weeks, K.L. \& McMullen, J.R. (2015) Pathophysiology of cardiac hypertrophy and heart failure: signaling pathways and novel therapeutic targets. Archives of toxicology, 89, 1401-1438.

van Berlo, J.H., Maillet, M. \& Molkentin, J.D. (2013) Signaling effectors underlying pathologic growth and remodeling of the heart. The Journal of clinical investigation, 123, 37-45.

Venetucci, L.A., Trafford, A.W., O'Neill, S.C. \& Eisner, D.A. (2008) The sarcoplasmic reticulum and arrhythmogenic calcium release. Cardiovascular Research, 77, 285-292.

Verheugt, C.L., Uiterwaal, C.S.P.M., van der Velde, E.T., Meijboom, F.J., Pieper, P.G., van Dijk, A.P.J., Vliegen, H.W., Grobbee, D.E. \& Mulder, B.J.M. (2010) Mortality in adult congenital heart disease. European heart journal, 31, 1220-1229.

Wagner, A. \& Wright, J. (2007) Alternative routes and mutational robustness in complex regulatory networks. Bio Systems, 88, 163-172.

Wang, J., Gareri, C. \& Rockman, H.A. (2018a) G-Protein-Coupled Receptors in Heart Disease. Circulation research, 123, 716-735.

Wang, J.-Y., Zhang, Y.-J., Li, H., Hu, X.-L., Li, M.-P., Song, P.-Y., Ma, Q.-L., Peng, L.-M. \& Chen, X.-P. (2018b) CRISPLD1 rs12115090 polymorphisms alters antiplatelet potency of clopidogrel in coronary artery disease patients in Chinese Han. Gene, 678, 226-232.

Watanabe, E., Yasui, K., Kamiya, K., Yamaguchi, T., Sakuma, I., Honjo, H., Ozaki, Y., Morimoto, S., Hishida, H. \& Kodama, I. (2007) Upregulation of KCNE1 induces QT interval prolongation in patients with chronic heart failure. Circulation journal : official journal of the Japanese Circulation Society, 71, 471-478.

Weeks, K.L. \& McMullen, J.R. (2011) The athlete's heart vs. the failing heart: can signaling explain the two distinct outcomes? Physiology (Bethesda, Md.), 26, 97-105.

Wei, S., Guo, A., Chen, B., Kutschke, W., Xie, Y.-P., Zimmerman, K., Weiss, R.M., Anderson, M.E., Cheng, H. \& Song, L.-S. (2010) T-tubule remodeling during transition from hypertrophy to heart failure. Circulation research, 107, 520-531.

WHO (2020) WHO reveals leading causes of death and disability worldwide: 2000-2019, Geneva, Switzerland.

Wilson, R., Golub, S.B., Rowley, L., Angelucci, C., Karpievitch, Y.V., Bateman, J.F. \& Fosang, A.J. (2016) Novel Elements of the Chondrocyte Stress Response Identified Using an in Vitro Model of Mouse Cartilage Degradation. Journal of proteome research, 15, 10331050.

Wu, Y., Roden, D.M. \& Anderson, M.E. (1999) Calmodulin kinase inhibition prevents development of the arrhythmogenic transient inward current. Circulation research, 84, 906-912. 
Wu, Y., Temple, J., Zhang, R., Dzhura, I., Zhang, W., Trimble, R., Roden, D.M., Passier, R., Olson, E.N., Colbran, R.J. \& Anderson, M.E. (2002) Calmodulin kinase II and arrhythmias in a mouse model of cardiac hypertrophy. Circulation, 106, 1288-1293.

Zapalska-Sozoniuk, M., Chrobak, L., Kowalczyk, K. \& Kankofer, M. (2019) Is it useful to use several "omics" for obtaining valuable results? Molecular biology reports, 46, 3597-3606.

Zhang, B., Li, B., Sun, C., Tu, T., Xiao, Y. \& Liu, Q. (2021) Identification of key gene modules and pathways of human platelet transcriptome in acute myocardial infarction patients through co-expression network. American journal of translational research, 13, 38903905.

Zhang, T., Maier, L.S., Dalton, N.D., Miyamoto, S., Ross, J., Bers, D.M. \& Brown, J.H. (2003) The deltaC isoform of CaMKII is activated in cardiac hypertrophy and induces dilated cardiomyopathy and heart failure. Circulation research, 92, 912-919.

Ziaeian, B. \& Fonarow, G.C. (2016) Epidemiology and aetiology of heart failure. Nature reviews. Cardiology, 13, 368-378. 


\section{Acknowledgements}

First and foremost, I want to thank Prof. Hasenfuß for the opportunity to work in his laboratory on this exciting project with all the freedom and trust to work autonomously. I learned a lot during the past years.

I further want to thank Sara Klas for her support and ideas during the first 1.5 years, for giving me the opportunity to start a new lab together and to help me in the beginning of my scientific career.

Additionally, I want to thank Karl Toischer for providing his support, for all his advice and for his effort that helped me a lot to finish my PhD thesis.

I would also like to thank my thesis committee members Bernd Wollnik, Dörthe Katschinski, George Kensah and my former thesis committee member Blanche Schwappach for their support during this project with their knowledge and scientific criticism in our meetings. Further, I thank Katrin Streckfuß-Bömeke, Laura Zelarayan and Ralf Dressel for joining the extended examination board.

I am very grateful to all the former members of the Hasenfuß lab for the nice working atmosphere. In particular, I would like to thank Anke Bode and Sarah Nourmohammadi for all the work they did so that I could concentrate on my research and for all the nice and funny talks we had.

My warm thanks go to Lukas Cyganek and his lab. Thank you for teaching me all about iPSC culture.

I also want to thank Timo Schulte for showing me all the mouse work and for providing help whenever needed and Sabrina Koszewa for taking care of my mouse lines when I was on parental leave.

I want to express my thanks to the whole SFB1002 Service Unit consisting of Sabrina Koszewa Blume, and Marcel Zoremba for the excellent job with all the echoes.

I further want to thank my parents and my sister Patricia for their constant support.

My final thanks go to my husband Robin and my wonderful daughter Hanna who always remind me of the important things in life. Thank you for your ongoing encouragement and support to finish this thesis. 